\title{
Equivariant spectral decomposition for flows with a $\mathbb{Z}$-action
}

\author{
LEE MOSHER \\ Institute for Advanced Studies $\dagger$, and CUNY Graduate School $\ddagger$, \\ New York, 10036, USA
}

(Received 12 November 1987 and revised 14 March 1988)

\begin{abstract}
Given a manifold $\tilde{M}$ equipped with a free, properly discontinuous, cocompact $\mathbb{Z}$-action, and a flow $\tilde{\phi}$ on $\tilde{M}$ which is $\mathbb{Z}$-equivariant, we study the qualitative dynamics of $\tilde{\phi}$. Under certain hypotheses on $\tilde{\phi}$, we show that the chain recurrent set of $\tilde{\phi}$ has a decomposition which is the analogue, in the category of $\mathbb{Z}$-equivariant flows, of Smale's spectral decomposition for recurrent sets of Axiom $A$ flows.
\end{abstract}

Given a compact manifold $M$, a flow $\phi$ on $M$, and a cohomology class $\alpha \in H^{1}(M ; \mathbb{Z})$, consider the $\mathbb{Z}$-covering $\tilde{M} \rightarrow M$ associated with $\alpha$, and the lifted flow $\tilde{\phi}$ on $\tilde{M}$. What can one say about the qualitative dynamics of $\tilde{\phi}$ ?

In a certain sense, this question is equivalent to the question of how the qualitative dynamics of $\phi$ are related to the cohomology class $\alpha$. For example, in the Schwartzman-Fried theory of cross sections of flows (see [F2]), it is shown that $\phi$ has a cross-section Poincare dual to the cohomology class $\alpha$ if and only if $\tilde{\phi}$ is a product flow, that is, $\tilde{M} \approx S \times \mathbb{R}$ for some compact manifold $S$, and $\tilde{\phi}$ is the flow in the $\mathbb{R}$ direction.

Although our question about qualitative dynamics is posed in the somewhat unfamiliar setting of a non-compact space $\tilde{M}, \tilde{\phi}$ can be extended to a flow $\tilde{\phi}^{\text {c }}$ on the end compactification $\tilde{M}^{\mathrm{c}}=\tilde{M} \cup\{-\infty,+\infty\}$, by letting $-\infty$ and $+\infty$ be stationary points. In the special case when $\tilde{\phi}$ is a product flow, notice that the chain recurrent set $R\left(\tilde{\phi}^{\mathrm{c}}\right)$ of the compactified flow $\tilde{\phi}^{\text {c }}$ consists exactly of the two points $\{-\infty,+\infty\}$, each of which forms a separate component of $R\left(\tilde{\phi}^{\mathrm{c}}\right)$. This is the simplest possible behavior for a compact flow. At the other extreme, one would expect the behavior of $\tilde{\phi}^{\mathrm{c}}$ to be very opaque if $-\infty$ and $+\infty$ were in the same component of $R\left(\tilde{\phi}^{\mathrm{c}}\right)$ : there might be orbits going from $-\infty$ to $+\infty$ and other orbits going back again, doing very strange things in the middle. We shall avoid this case altogether; that is, we shall consider only those flows $\tilde{\phi}$ such that $-\infty$ and $+\infty$ are contained in separate components of the chain recurrent set of $\tilde{\phi}^{c}$.

This restriction on $\tilde{\phi}^{c}$ implies in turn a restriction on the cohomology class $\alpha$, which can be stated in terms of homology directions for the flow $\phi$. Homology directions were introduced by Fried in [F2]; they can be thought of as a version of 
the asymptotic cycles studied by Schwartzman [Sch]. Loosely speaking, a homology direction of $\phi$ is an element living in the projectivized homology space $H_{1}(M ; \mathbb{R}) / \mathbb{R}_{+} \approx\{$ sphere $\} \cup\{$ point $\}$ which is approximated by the projectivized homology classes of long, almost closed orbits of $\phi$. Although $\alpha$ cannot be evaluated on a homology direction $d$, it does make sense to ask whether $\alpha(d)$ is positive, negative, or zero. The main result of the Schwartzman-Fried theory is that $\tilde{\phi}$ is a product flow if and only if $\alpha$ is positive on all homology directions of $\phi$. It is a simple extension of Fried's arguments (Proposition 9.1) to show that if $-\infty$ and $+\infty$ are in separate chain components of $\tilde{\phi}^{\mathrm{c}}$, then $\alpha$ is non-negative on all homology directions of $\phi$.

Thus, under the hypothesis that $\alpha$ is non-negative on homology directions of $\phi$, we ask: what are the qualitative dynamics of $\tilde{\phi}$ ? We shall give a reasonably complete answer to this question for one special class of flows $\phi$, namely the pseudo-Anosov flows, i.e. those 3-dimensional flows which are suspensions of pseudo-Anosov maps of surfaces. Our main theorem, the $\mathbb{Z}$-Spectral Decomposition Theorem, lists several properties of $\tilde{\phi}$ in the case that $\phi$ is pseudo-Anosov. The most important property says that, although the chain recurrent set $R(\tilde{\phi})$ has infinitely many chain components, each chain component is compact, and there are only finitely many orbits of chain components under the action of $\mathbb{Z}$. This should be compared to the statement of the original Spectral Decomposition Theorem of Smale [Sm], which says that the recurrent set of an Axiom $\boldsymbol{A}$ flow has only finitely many components. We will also prove a property which shows that in a certain sense, $\tilde{\phi}$ is well-behaved near the ends $\pm \infty$ of $\tilde{M}$.

Our interest in the question of the qualitative dynamics of $\mathbb{Z}$-equivariant flows is motivated by an attempt to generalize the Schartzmann-Fried result, which classifies those cohomology classes $\alpha$ which are Poincaré dual to a cross-section to $\phi$. Recall that a cross-section to $\phi$ is a codimension-1 closed submanifold of $M$ transverse to $\phi$ (i.e. a 'transverse surface') which intersects every flow line. In a companion paper [M], we consider the following question: given a cohomology class $\alpha$, when is $\alpha$ Poincaré dual to a transverse surface $S$ to $\phi$ which does not necessarily hit every flow line? The lifted flow $\tilde{\phi}$ can be employed to shed some light on this problem: a necessary condition for the existence of $S$ is that the compactified flow $\tilde{\phi}^{\text {c }}$ satisfy our special hypothesis on $\pm \infty: S$ must lift to a compact transverse surface $\tilde{S}$ to $\tilde{\phi}$ which separates $-\infty$ from $+\infty$, so the two points $-\infty$ and $+\infty$ are in separate components of the chain recurrent set of $\tilde{\phi}^{\text {c }}$. Thus, $\alpha$ must be non-negative on homology directions of $\phi$.

The converse question is: given that $\alpha$ is non-negative on all homology directions, is there a transverse surface to $\phi$ Poincaré dual to $\alpha$ ? The course of argument proposed here is to learn as much as possible about the qualitative dynamics of $\tilde{\phi}$, and use this knowledge to construct the desired transverse surface. In this paper, our investigations cover one half of this course of argument: given that $\alpha$ is non-negative on all homology directions of $\phi$, we shall study the qualitative dynamics of $\tilde{\phi}$. As the results of Fried cover the case where $\alpha$ is positive on all homology directions, we are interested here only in the case where $\alpha$ takes on the value zero 
on some homology directions. In a companion paper [M], we use the results proven here to investigate the remaining half of the argument: in particular, in [M] we prove that when $\phi$ is a pseudo-Anosov flow and $\alpha$ is non-negative on the homology directions of $\phi$, then $\alpha$ is indeed Poincaré dual to some transverse surface to $\phi$.

Our interest in pseudo-Anosov flows was spurred by the results of [F1], which gave a flow-theoretic interpretation of certain results of Thurston on the structure of 3-manifolds which fiber over the circle. Thurston showed [T] that for a 'nice' 3-manifold $M$, there is a naturally defined norm $x$ on $H^{1}(M ; \mathbb{R})$ which is related to fiberings of $M$ over the circle. The unit ball $B_{x}$, instead of having the shape of an ellipsoid, is a polyhedron with integrally defined faces. Thurston also showed that if $\alpha \in H^{1}(M ; \mathbb{Z}) \subset H^{1}(M ; \mathbb{R})$ is Poincare dual to a fiber of some fibration over $S^{1}$, then there is a top-dimensional face $\sigma$ of $B_{x}$ such that $\alpha \in$ int (Cone $(\sigma)$ ), and every other integral class in int (Cone $(\sigma)$ ) is Poincaré dual to a fiber of some fibration; in this case, $\sigma$ is called a fibered face of the Thurston norm. Fried proved that for a fibered face $\sigma$ of the Thurston norm of an irreducible, atoroidal 3-manifold $M$, there exists a flow $\phi$ such that every integral class $\alpha \in$ int (Cone $(\sigma))$ is represented by a cross-section to $\phi$ whose first return map is pseudo-Anosov. In fact, Fried proved that int (Cone $(\sigma)$ ) consists precisely of those classes which are positive on all homology directions of the flow $\phi$. This shows that $\partial($ Cone $(\sigma))$ consists precisely of those classes $\alpha \in H^{1}(M ; \mathbb{R})$ which are non-negative on all homology directions of $\phi$ and zero on some homology directions of $\phi$; since the faces of $B_{x}$ are integrally defined, there exist such classes $\alpha$ which are integral. Our main result, the $\mathbb{Z}$-Spectral Decomposition Theorem, can therefore be interpreted as giving information about the boundary of a fibered face of Thurston's norm. In the companion paper [M], this interpretation is utilized to settle a question of Oertel [O], by showing that for a fibered face $\sigma$ of Thurston's norm, there exists a branched surface $\Sigma \subset M$ which carries representatives of every class in Cone $(\sigma)$.

Although we have restricted our discussion to pseudo-Anosov flows on 3-manifolds, our methods may also apply when $\phi$ is any flow with good symbolic dynamics, to yield a $\mathbb{Z}$-Spectral Decomposition Theorem in those cases. The proof we give of the $\mathbb{Z}$-Spectral Decomposition Theorem for pseudo-Anosov flows utilizes very strongly certain properties of Markov partitions, and in particular requires a good understanding of side identifications among the Markov flow boxes. In the pseudoAnosov case, these side identifications are easy to understand, and the proof goes through. There are probably some difficulties in generalizing this proof to the case of higher dimensional basic sets of Axiom $A$ flows, because of the complicated side identification among the Markov flow boxes. On the other hand, our methods do apply to a 1-dimensional basic set of an Axiom $A$ flow $\phi$ on a manifold $M$, where according to Bowen [B1] there is a Markov partition whose flow boxes have no boundary identifications at all. In the final section, we shall describe briefly how a $\mathbb{Z}$-Spectral Decomposition Theorem can be formulated and proven for the 1dimensional case. For general basic sets, we show how the theorem can be reduced to a conjecture concerning the existence of isolating blocks of hyperbolic invariant sets possessing nice homological properties. 
As our main interest is in applications to the topology of 3-manifolds, we shall for the most part concentrate entirely on the case of pseudo-Anosov flows. In fact, this case has some interesting difficulties that do not appear in the Axiom $A$ case, because of the failure of standard shadowing arguments.

The statement of the $\mathbb{Z}$-Spectral Decomposition Theorem is given in $\S 0$, together with some preliminary definitions. The proof of the theorem occupies $\$ \$ 1-8$; the content of these sections is described briefly at the end of $\S 0$. $\S 9$ contains a few comments about extending the $\mathbb{Z}$-Spectral Decomposition Theorem to the case of basic sets of Axiom $A$ flows.

\section{Preliminaries and statement of the $\mathbb{Z}$-Spectral Decomposition Theorem}

First we review some basic notions concerning pseudo-Anosov maps. The standard reference for pseudo-Anosov maps is [FLP]; reader is referred there for more detailed definitions and basic results.

Given a closed, oriented surface $S$ and an orientation preserving homeomorphism $f: S \rightarrow S, f$ is pseudo-Anosov if there exists $\lambda>1$ and a pair of transverse measured foliations $\mathscr{F}_{u}$ and $\mathscr{F}_{s}$ which fill up $S$ such that $f\left(\mathscr{F}_{s}\right)=\lambda^{-1} \cdot \mathscr{F}_{s}$ and $f\left(\mathscr{F}_{u}\right)=\lambda \cdot \mathscr{F}_{u}$. Given a point $s \in S$ and $n \geq 3, s$ is an $n$-pronged singularity of $\mathscr{F}_{s}$ if and only if $s$ is an $n$-pronged singularity of $\mathscr{F}_{u}$; in this case, we say that $s$ is an $n$-pronged singularity of $f$, and we write $\operatorname{sing}(f, n)=\{s \in S \mid s$ is an $n$-pronged singularity of $f\}$. Also, we write $\operatorname{sing}(f)=\bigcup_{n \geq 3} \operatorname{sing}(f, n)$.

The mapping torus of $f$ is the 3-manifold $M$ obtained from $S \times I$ by gluing $(x, 1)$ to $(f(x), 0)$ for all $x \in S$. The suspension flow of $f$ is the flow $\phi$ on $M$ induced by the semi-flow $(x, s) \cdot t=(x, s+t)$ on $S \times I$, where $0 \leq s, t, s+t \leq 1$. Henceforth, for any $x \in M$ and $t \in \mathbb{R}$, we shall write $x \cdot t$ as a shorthand for $\phi_{t}(x)$; also, for any interval $[a, b]$ in $\mathbb{R}$, we shall write $x \cdot[a, b]$ for the flow segment $\{x \cdot t \mid t \in[a, b]\}$.

Now we review Fried's theory of homology directions for a flow $\phi$ on a compact manifold $M$; details can be obtained in [F2]. Given $x \in M$, a closing sequence based at $x$ is a sequence $\left(x_{i}, t_{i}\right)$ such that $x_{i} \rightarrow x, t_{i} \rightarrow \infty$ and $d\left(x_{i}, x_{i} \cdot t_{i}\right)<\varepsilon_{i}$ where $\varepsilon_{i} \rightarrow 0$ as $i \rightarrow \infty$. When $i$ is large enough, the fundamental group of each $\varepsilon_{i}$ ball $B\left(x_{i}, \varepsilon_{i}\right)$ injects trivially into $\pi_{1}(M)$. Thus, there is a well-defined homology class $c_{i} \in$ $H_{1}(M ; \mathbb{R})$ obtained by concatenating the flow segment $x_{i} \cdot\left[0, t_{i}\right]$ with a path from $x_{i} \cdot t_{i}$ to $x_{i}$ staying in $B\left(x_{i}, \varepsilon_{i}\right)$. Let $p\left(c_{i}\right)$ denote the projective image of $c_{i}$ in $H_{1}(M ; \mathbb{R}) / \mathbb{R}_{+}$, where the latter space is topologized as a sphere disjoint union a point. Any limit point of the sequence $p\left(c_{i}\right)$ as $i \rightarrow \infty$ is called a homology direction for the flow $\phi$. The set of all homology directions forms a set $D_{\phi} \subset H_{1}(M ; \mathbb{R}) / \mathbb{R}_{+}$. Given a set $A \subset H_{1}(M ; \mathbb{R}) / \mathbb{R}_{+}$, let Cone' $(A)$ denote the smallest closed convex cone containing the inverse image of $A$ under the projection map $p: H_{1}(M ; \mathbb{R}) \rightarrow$ $H_{1}(M ; \mathbb{P}) / \mathbb{R}_{+}$, and let Cone $(A)$ be Cone' $(A)-p^{-1}(A \cap\{0\})$. In other words, if $A$ does not contain the point $p(0)$, then Cone $(A)$ is obtained from Cone' $(A)$ by deleting the origin; otherwise, Cone $(A)=$ Cone' $^{\prime}(A)$. Given $\alpha \in H^{1}(M ; \mathbb{R})$ and $p(c) \in H_{1}(M ; \mathbb{R}) / \mathbb{R}_{+}$, the intersection sign

$$
\alpha(p(c))=\operatorname{sign}(\alpha(c)) \in\{-1,0,1\}
$$

is well-defined, independent of the choice of $c$ representing $p(c)$. 
Cross-Section Theorem (Fried). Given $\alpha \in H^{1}(M ; \mathbb{Z}), \alpha$ is Poincaré dual to a cross-section to $\phi$ if and only if $\alpha(d)>0$ for all $d \in D_{\phi}$.

Next we describe the Thurston norm on the homology of a 3-manifold (see [T] for details), and Fried's results [F1] concerning fibered faces of the Thurston norm. The Thurston norm $x$ on $H^{1}(M ; \mathbb{R})$ is defined as follows. Given a compact surface $A$, if $A$ is connected define $\chi_{-}(A)=\max \{0,-\chi(A)\}$, and if $A$ is a union of connected components $A=A_{1} \cup \cdots \cup A_{k}$ define $\chi_{-}(A)=\chi_{-}\left(A_{1}\right)+\cdots+\chi_{-}\left(A_{k}\right)$. Given $\alpha \in$ $H^{1}(M, \mathbb{Z}), x(\alpha)$ is the minimum, over all embedded surfaces $A \subset M$ Poincaré dual to $\alpha$, of $\chi_{-}(A)$. Thurston showed that $x$ extends by homogeneity and continuity to a semi-norm on $H^{1}(M ; \mathbb{R})$, which is a norm when $M$ is irreducible and atoroidal (we shall consider $H^{1}(M ; \mathbb{Z})$ to be embedded in $H^{1}(M ; \mathbb{R})$ in the natural way). Moreover, he proved the following theorem describing the structure of the unit ball $B_{x}$ of $x$.

THEOREM (Thurston). The Thurston norm $x$ is the supremum of finitely many integrally defined linear functionals on $H^{1}(M ; \mathbb{R})$. In particular, the unit ball $B_{x}$ of $x$ is a finite polyhedron with integrally defined faces. Moreover, there is a specific set of topdimensional faces of $B_{x}$, called fibered faces, with the property that an integral cohomology class $\alpha \in H^{1}(M ; \mathbb{Z})$ is Poincaré dual to a fiber of a fibration $M \rightarrow S^{1}$ if and only if $\alpha \in$ int (Cone $(\sigma))$ for some fibered face $\sigma$ of $B_{x}$.

Fried has shown that there is a connection between the fibered faces of $B_{x}$ and certain non-singular flows on $M$ :

TheOREM (Fried). Suppose $M$ is irreducible and atoroidal. There is a natural way to associate, to each fibered face $\sigma$ of $B_{x}$, a non-singular flow $\phi$ on $M$ with the following property: for each class $\alpha \in H^{1}(M, \mathbb{Z}), \alpha$ is Poincare dual to a cross-section $A$ of $\phi$ if and only if $\alpha \in$ int (Cone $(\sigma))$, in which case the first return map of $\phi$ to $A$ is pseudo-Anosov. $\phi$ is uniquely characterized by this property, up to isotopy and reparameterization.

The uniqueness property can be restated by saying that the oriented 1-dimensional foliation underlying $\phi$ is unique up to isotopy. We say that $\phi$ is a pseudo-Anosov flow associated with the face $\sigma$. In particular, consider a pseudo-Anosov map $f: S \rightarrow S$ with mapping torus $M$ and suspension flow $\phi$; the quotient map $S \times I \rightarrow M$ determines a fibration $q: M \rightarrow S^{1}$ with fiber $S \times 0$, so the Poincare dual of $\alpha$ of $S \times 0$ is contained in int (Cone $(\sigma)$ ) for some fibered face $\sigma$ of $B_{x}(M)$. Fried's theorem says that for any other fibration $q^{\prime}: M \rightarrow S^{1}$, with fiber $S^{\prime}$, pseudo-Anosov monodromy map $f^{\prime}$, and suspension flow $\phi^{\prime}$ on $M$, if $S^{\prime}$ is Poincaré dual to an element of int (Cone $(\sigma)$ ), then $\phi$ and $\phi^{\prime}$ are isotopic, up to reparameterization.

Combining the above theorem with the cross-section theorem, we arrive at:

Corollary: Fried Duality for Pseudo-Anosov Flows. Let $\phi$ be a pseudoAnosov flow associated with a fibered face $\sigma$ of an irreducible, atoroidal manifold $M^{3}$. Then Cone $(\sigma)$ is the dual cone to Cone $\left(D_{\phi}\right)$. In other words, Cone $(\sigma)=$ 
$\left\{\alpha \in H^{1}(M ; \mathbb{R}) \mid \alpha(d) \geq 0\right.$ for all $\left.d \in D_{\phi}\right\}$. In particular, given a face $F$ of $\partial($ Cone $(\sigma))$, if we define the dual face $F^{*}=\left\{\alpha \in\right.$ Cone $\left.\left(D_{\phi}\right) \mid \alpha(F)=0\right\}$, then the correspondence $F \rightarrow F^{*}$ is a 1-1 correspondence between faces of $\partial($ Cone $(\sigma))$ and faces of $\partial\left(\right.$ Cone $\left.\left(D_{\phi}\right)\right)$.

Our main theorem, the $\mathbb{Z}$-Spectral Decomposition Theorem, will show how the flow $\phi$ is related to a class $\alpha \in \partial($ Cone $(\sigma)) \cap H^{1}(M ; \mathbb{Z})$. We shall be concerned with the qualitative dynamics of the lifted flow $\tilde{\phi}$ on the $\mathbb{Z}$-cover $\tilde{M}$ of $M$ determined by $\alpha$. In order to state the theorem, we shall need some generalities used in the study of the qualitative dynamics of flows; a good reference is Conley's book [C].

Let a fixed flow $\Phi$ on a metric space $N$ be given, with metric $d$. For our main application, $N$ will be the space $\tilde{M}$ and $d$ will be a $\mathbb{Z}$-equivariant metric. Given a point $x \in N, L_{+}(x)$, also known in the literature as $\omega(x)$, is the set of all limit points of sequences of the form $x \cdot t_{i}$, where $t_{i} \rightarrow+\infty$ as $i \rightarrow+\infty ; L_{-}(x)$, also known as $\alpha(x)$, is defined similarly by letting $t_{i} \rightarrow-\infty$. Given a set $X \subset N$, we shall also occasionally make use of the notation $L_{+}(X)=\bigcup\left\{L_{+}(x) \mid x \in X\right\}$, and similarly for $L_{-}(X)$. Given $\varepsilon, T>0$, an $\varepsilon$, $T$ chain is a pair of sequences $x_{0}, x_{1}, \ldots, x_{n} \in N, t_{1}, \ldots, t_{n} \in \mathbb{R}_{+}$, such that for $1 \leq i \leq n, t_{i}>T$ and $d\left(x_{i-1} \cdot t_{i}, x_{i}\right)<\varepsilon$; this chain is said to go from $x_{0}$ to $x_{n}$. If $x_{0}=x_{n}$, this is called an $\varepsilon, T$ cycle through $x_{0}$. Given $x \in N, R_{+}(x)$ is defined as the set of all points $y$ such that for all $\varepsilon, T>0$, there exists an $\varepsilon, T$ chain from $x$ to $y ; R_{-}(x)$ is similarly defined by taking chains ending at $x$. The chain recurrent set $R=R(\Phi)$ is the set of all points $x \in N$ such that $x \in R_{+}(x)$, i.e. there exists an $\varepsilon, T$ cycle through $x$ for all $\varepsilon, T>0$. It is a fact that the restriction of $\Phi$ to $R$ is a chain recurrent flow, i.e. the chain recurrent set of $\Phi \mid R$ is all of $R$. A closed invariant set $C$ of $\Phi$ is chain connected or chain transitive if for any $x, y \in C$, there exists an $\varepsilon, T$ chain from $x$ to $y$ for all $\varepsilon, T>0$. In general, given any closed invariant set $I$ of $\Phi$ such that $\Phi \mid I$ is a chain recurrent flow, a closed invariant subset $C \subset I$ is called a chain component of $I$ if it is a maximal chain connected set. It is evident that the collection of chain components of $I$ is a partition of the set $I$.

$\mathbb{Z}$-Spectral Decomposition Theorem. Given a manifold $M^{3}$, a pseudo-Anosov flow $\phi$ on $M$, and a primitive element $\alpha \in H^{1}(M ; \mathbb{Z})$, suppose that $\alpha(d) \geq 0$ for all $d \in D_{\phi}$, and $\alpha(d)=0$ for some $d \in D_{\phi}$. Let $\tilde{M} \rightarrow M$ be the $\mathbb{Z}$-cover defined by $\alpha$, and let $\tilde{\phi}$ be the flow on $\tilde{M}$ which lifts $\phi$. Let $R$ be the chain recurrent set of $\tilde{\phi}$. Then:

(A) Each chain component of $R$ is compact.

(B) There are finitely many orbits of chain components of $R$ under the action of $\mathbb{Z}$.

(C) For any $x \in \tilde{M}-R$, either $L_{+}(x)=\{+\infty\}$ or $L_{+}(x)$ is contained in some chain component of $R$.

(D) Similarly, for any $x \in \tilde{M}-R$, either $L_{-}(x)=\{-\infty\}$ or $L_{-}(x)$ is contained in some chain component of $R$.

(E) If $L_{+}(x) \neq\{+\infty\}$, then there exists a neighborhood $U$ of $+\infty$ such that for any chain component $C$ of $R$, if $C \subset U$ then $C \subset R_{+}(x)$; a similar statement holds when $L_{-}(x) \neq\{-\infty\}$. 
Because of Fried Duality for Pseudo-Anosov Flows, the hypothesis on $\alpha$ can be restated as follows: if $\sigma$ is the fibered face of $B_{x}(M)$ which is associated with the pseudo-Anosov flow $\phi$, then $\alpha \in H^{1}(M ; \mathbb{Z}) \cap \partial($ cone $(\sigma))$. To see that this hypothesis is non-vacuous, notice that because of Thurston's theorem the faces of $B_{x}(M)$ are integrally defined, so for any sub-face $\sigma^{\prime} \subset \sigma, H^{\prime}(M ; \mathbb{Z}) \cap$ int (cone $\left.\left(\sigma^{\prime}\right)\right) \neq \varnothing$. This also follows from Fried's description of Cone $(D \phi)$; see Theorem 3.4.

Besides properties (A) and (B), the most interesting property is (E). This says that, in a certain sense, the flow is well-behaved near the ends of $\tilde{M}$. This property is crucial in the construction, given in the companion paper [M], of transverse surfaces to $\tilde{\phi}$ which separate $-\infty$ from $+\infty$. As a consequence of property (E), it is possible to prove that the chain recurrent set of the compactified flow $\tilde{\phi}^{\mathrm{c}}$ is the union of the chain recurrent set of $\bar{\phi}$ with the set of ends $\{-\infty,+\infty\}$.

The proof of the $\mathbb{Z}$-Spectral Decomposition theorem is presented in $\S \S 1-8$, whose contents are briefly described here.

In $\S 1$, the proof is reduced to the case of a pseudo-Anosov $f$ which fixes all its separatrices.

In $\S 2$, we review the symbolic dynamics of pseudo-Anosov maps and flows.

In $\S 3$, the main body of the proof begins. We introduce the main construction used in the proof, the 'chain kernel' of $\alpha$. This is a closed invariant subset of the flow $\phi$, defined as the set of points $x \in M$ such that for all $\varepsilon, T>0$, there exists an $\varepsilon, T$ cycle $X$ through $x$ with $\alpha(X)=0$. Most of the proof is devoted to studying properties of the chain kernel of $\alpha$, with the aim of showing that the total lift of the chain kernel to $\tilde{M}$ is the same as the chain recurrent set of $\tilde{\phi}$.

Also in $\S 3$, we introduce a notion which is designed to overcome certain difficulties inherent in the study of pseudo-Anosovs. The main difficulty is that the standard methods of shadowing theory do not apply: because of the presence of singularities in pseudo-Anosov maps, pseudo-Anosov flows have $\varepsilon, T$ cycles which are not shadowed by any periodic orbits. In order to overcome this difficulty we introduce the notion of a 'quasi-orbit'; heuristically, a quasi-orbit is a finite number of true orbits of the flow that, taken collectively, can be used to shadow $\varepsilon, T$ cycles.

In $\S 4$, we study the symbolic dynamics of quasi-orbits.

In $\S 5$, we continue the study of quasi-orbits, and we develop a shadowing theory for pseudo-Anosovs, which is used to characterize the chain kernel of $\alpha$. As a consequence, we prove that the chain kernel has only finitely many chain components, which is the main step in proving property (B). Shadowing theory for pseudo-Anosovs was introduced by Handel [H1], [H2], who used it to understand the entropy of surface maps in the isotopy class of a pseudo-Anosov. Our approach to pseudo-Anosov shadowing theory is motivated by Handel's theory, although our proofs follow along the more classical lines contained in Shub's book [Sh].

In $\S 6$, we show how each chain component of the chain kernel of $\alpha$ can be lifted homeomorphically to $\tilde{M}$, by using isolating blocks.

In $\S 7$, we study the transient behavior of orbits of $\tilde{\phi}$, and we prove that the total lift of the chain kernel of $\alpha$ is, in fact, the chain recurrent set of $\tilde{\phi}$. This section contains the proofs of properties (A)-(D) of the theorem. 
In $\S 8$, we study the behavior of $\tilde{\phi}$ near the ends of $\tilde{M}$, and prove property (E) of the theorem.

In $\S 9$, we give a short discussion of generalizations of the $\mathbb{Z}$-Spectral Decomposition Theorem to the case of basic sets of Axiom $A$ flows.

\section{Reduction to case of fixed separatrices}

There are many places in the proof of the $\mathbb{Z}$-Spectral Decomposition Theorem where it will be convenient to assume that the flow $\phi$ is a suspension of a pseudo-Anosov map $f$ which acts as the trivial permutation on its singular separatrices. Although this assumption could be dispensed with at the cost of more intricate arguments, it is easier to reduce the proof of the theorem to the case of fixed separatrices. We begin by demonstrating this reduction.

Let $f$ be an arbitrary pseudo-Anosov map, $M$ the mapping torus of $f, \phi$ the suspension flow on $M$, and $k$ the least power such that $f^{k}$ fixes separatrices. Let $N$ be the mapping torus of $f^{k}, \psi$ the suspension flow of $f^{k}$ on $N$. There is a $\mathbb{Z} / k$ covering map $p: N \rightarrow M$ corresponding to the kernel of the homomorphism $\pi_{1}(M) \rightarrow$ $\mathbb{Z} \rightarrow \mathbb{Z} / k$ where $\pi_{1}(M) \rightarrow \mathbb{Z}$ is the induced homomorphism of the fibration $M \rightarrow S^{1}$ corresponding to $f . \psi$ is the pull-back flow of $\phi$ under $p: N \rightarrow M$.

We shall show that for any $\alpha \in H^{1}(M ; \mathbb{Z})$, the hypotheses of the theorem for $M$, $\phi$, and $\alpha$ imply the hypotheses of the theorem for $N, \psi$, and $p^{*}(\alpha) \in H^{1}(N ; \mathbb{Z})$. Then we shall show that the conclusions of the theorem for $N, \psi$, and $p^{*}(\alpha)$ imply the conclusions of the theorem for $M, \phi$, and $\alpha$. Note that the map $p^{*}: H^{1}(M ; \mathbb{R}) \rightarrow$ $H^{1}(N ; \mathbb{R})$ is an injection, and the restriction $p^{*}: H^{1}(M ; \mathbb{Z}) \rightarrow H^{1}(N ; \mathbb{Z})$ takes primitive classes to primitive classes.

Consider the cones of homology directions Cone $\left(D_{\phi}\right) \subset H_{1}(M ; \mathbb{R})$, Cone $\left(D_{\psi}\right) \subset$ $H_{1}(N ; \mathbb{R})$, and the dual cones $C_{\phi} \subset H^{1}(M ; \mathbb{R}), C_{\psi} \subset H^{1}(N ; \mathbb{R})$. We claim that, under the injection $p^{*}: H^{1}(M ; \mathbb{R}) \rightarrow H^{1}(N ; \mathbb{R}), p^{*}\left(C_{\phi}\right)=C_{\psi} \cap$ image $\left(p^{*}\right)$. Since a crosssection of $\phi$ lifts to a cross-section of $\psi$, the inclusion in one direction is obvious. Conversely, suppose that $\beta \in$ int $\left(C_{\psi}\right) \cap$ image $\left(p^{*}\right)$. Choose a closed 1 -form $\omega$ representing $\beta$ such that $\omega$ is positive on flow lines of $\psi$. Since image $\left(p^{*}\right)$ is pointwise invariant under the action of $\mathbb{Z} / K$ on $H^{1}(M ; \mathbb{R})$, then the average of $\omega$ over $\mathbb{Z} / K$ is a closed 1-form $\omega^{\prime}$, positive on flow lines of $\psi$, invariant under $\mathbb{Z} / K$, and representing the cohomology class $\beta$. Thus, $\omega^{\prime}$ is the pull-back of a 1 -form on $M$, positive on flow lines of $\phi$. Thus, $\beta \in p^{*}\left(C_{\phi}\right)$.

From the previous paragraph, it follows that if $\alpha \in \partial\left(C_{\phi}\right)$, then $p^{*}(\alpha) \in \partial\left(C_{\psi}\right)$. It follows that the hypotheses of the theorem for $M, \phi$, and $\alpha$ imply the hypotheses of the theorem for $N, \psi$, and $p^{*}(\alpha)$.

Now let $\tilde{N} \rightarrow N$ be the $\mathbb{Z}$-cover associated to $p^{*}(\alpha) \in H^{1}(N ; \mathbb{Z})$ and let $\tilde{\psi}$ be the lift of $\psi$, and we assume that the conclusions of the theorem hold for $\tilde{\psi}$. By an analysis of fundamental groups, we shall now show that there is a $\mathbb{Z} / k$ covering map $\tilde{p}: \tilde{N} \rightarrow \tilde{M}$ making diagram 1 commute:

Let $G=\pi_{1}(M)$; the primitive cohomology class $\alpha \in H^{1}(M ; \mathbb{Z})$ defines an epimorphism $\alpha: G \rightarrow \mathbb{Z}$, and $K=\operatorname{ker}(\alpha)$ is canonically identified with $\pi_{1}(\tilde{M})$. Consider also the surjective homomorphism $\beta: G \rightarrow \mathbb{Z} / k$ associated with the covering map 


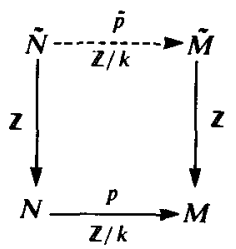

Diagram 1.

$N \rightarrow M$. Let $H=\operatorname{ker}(\beta)$; then $H$ is canonically identified with $\pi_{1}(N)$. The cohomology class $p^{*}(\alpha) \in H^{1}(N ; \mathbb{Z})$ associated with the covering $\tilde{N} \rightarrow N$ defines an epimorphism $p^{*}(\alpha): H \rightarrow \mathbb{Z}$, which is evidently the same as the restricted map $\alpha \mid H: H \rightarrow \mathbb{Z}$. Set $F=\operatorname{ker}(\alpha \mid H) \subset H$, so that $F$ is canonically identified with $\pi_{1}(\tilde{N})$. It is evident that

$F=\operatorname{ker}(\alpha \mid H)=\operatorname{ker}(\alpha \mid \operatorname{ker}(\beta))=\operatorname{ker}(\alpha) \cap \operatorname{ker}(\beta)=\operatorname{ker}(\beta \mid \operatorname{ker}(\alpha))=\operatorname{ker}(\beta \mid K)$, and since $\beta \mid K: K \rightarrow \mathbb{Z} / k$, this proves the existence of the required $\mathbb{Z} / k$ covering map $\tilde{p}: \tilde{N} \rightarrow \tilde{M}$. This discussion is summarized in the accompanying commutative diagram of subgroups and epimorphisms of $\pi_{1}(M)$, in which every linear subsequence is exact (see diagram 2 ).

It is evident that $\tilde{p}$ respects $\mathbb{Z}$-actions, and that $\tilde{\psi}$ is the lifted flow of $\tilde{\phi}$ under $\tilde{p}$. Note that $\tilde{p}$ maps $\varepsilon, T$ cycles of $\tilde{\psi}$ to $\varepsilon, T$ cycles of $\tilde{\phi}$, so $\tilde{p}$ takes $R(\tilde{\psi})$ to $R(\tilde{\phi})$. Moreover, this map is surjective, since each $\varepsilon, T$ chain of $\tilde{\phi}$ pulls back to an $\varepsilon, T$ chain of $\tilde{\psi}$. Since chain connected sets go to chain connected sets, each chain component of $R(\tilde{\psi})$ maps surjectively to a chain component of $R(\tilde{\phi})$; property (A) of the $\mathbb{Z}$-Spectral Decomposition Theorem says that chain components are compact, so property (A) for $\tilde{\psi}$ directly implies property (A) for $\tilde{\phi}$. Moreover, letting $\mathscr{C}(R)$ denote the collection of chain components of the chain recurrent set $R$, there is an induced map taking $\mathscr{C}(R(\tilde{\psi}))$ onto $\mathscr{C}(R(\tilde{\phi}))$ which respects $\mathbb{Z}$-actions; property (B) says that there are only finitely many $\mathbb{Z}$-orbits of chain components, so property (B) for $\tilde{\phi}$ follows from property (B) for $\tilde{\psi}$.

Given $x \in \tilde{M}-R(\tilde{\phi})$, consider $L_{+}(x)$. Choosing $y \in \tilde{p}^{-1}(x)$, clearly $y \in \tilde{N}-R(\tilde{\psi})$, and $\tilde{p}\left(L_{+}(y)\right)=L_{+}(x)$. Thus, property (C) for $\tilde{\phi}$ follows directly from property (C) for $\tilde{\psi}$; a similar statement holds for property (D).

When $R$ is the chain recurrent set of a flow, there is a natural acyclic partial order on $\mathscr{C}(R)$ defined as follows: if $C \neq C^{\prime} \in \mathscr{C}(R)$, then $C<C^{\prime}$ if there exists a sequence $C=C_{0}, C_{1}, \ldots, C_{k}=C^{\prime}$ in $\mathscr{C}(R)$ and a sequence $x_{1}, \ldots, x_{k}$ in the complement of

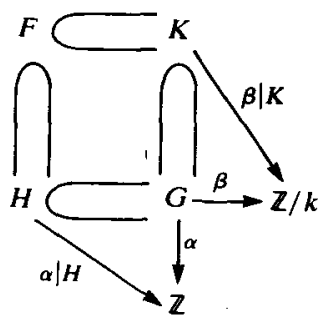

Diagram 2. 
$R$, such that $L_{-}\left(x_{i}\right) \subset C_{i-1}$ and $L_{+}\left(x_{i}\right) \subset C_{i}$. Property (E) is equivalent to the following statement for the partially ordered set $\mathscr{C}=\mathscr{C}(R(\tilde{\phi}))$ :

Property (E'). For every $C \in \mathscr{C}$, there exists a subset $V$ of $\mathscr{C}$ such that $C^{\prime} \in V$ implies $C<C^{\prime}$ ', and $V$ is a 'neighborhood of $+\infty$ ' in the sense that every increasing sequence in $\mathscr{C}$ is eventually contained in $V$.

The equivalence of property $(E)$ and property $\left(E^{\prime}\right)$ is easy to establish; for a detailed proof, the reader is referred to Proposition 8.1.

By naturality of the chain recurrent set, $\mathbb{Z} / \mathrm{k}$ acts on $\mathscr{C}(R(\tilde{\psi}))$; also by naturality, $\mathbb{Z} / \boldsymbol{k}$ preserves the partial order on $\mathscr{C}(R(\tilde{\psi}))$. Since the group $\mathbb{Z} / k$ has finite order, and since $\mathscr{C}(R(\tilde{\psi}))$ is acyclic, the different elements of $\mathscr{C}(R(\tilde{\psi}))$ in a given $\mathbb{Z} / \boldsymbol{k}$ orbit are non-comparable. Thus, the quotient $\mathscr{C}(R(\tilde{\psi})) /(\mathbb{Z} / k)$ inherits an acyclic partial ordering. Property (E') for $\mathscr{C}=\mathscr{C}(R(\tilde{\psi}))$ evidently implies property (E') for $\mathscr{C}=\mathscr{C}(R(\tilde{\psi})) /(\mathbb{Z} / k)$. Notice that the natural bijection $\mathscr{C}(R(\tilde{\psi})) /(\mathbb{Z} / k) \rightarrow \mathscr{C}(\boldsymbol{R}(\tilde{\phi}))$ is order preserving. Thus, property $\left(E^{\prime}\right)$ holds for $\mathscr{C}=\mathscr{C}(R(\tilde{\phi}))$.

\section{Symbolic dynamics}

Following the methods in [FLP], we give a description of the symbolic dynamics of a pseudo-Anosov map. No proofs are given, and the basic definitions are assumed; the reader is referred to [FLP].

Let $f: S \rightarrow S$ be a pseudo-Anosov map on a compact, connected, orientable surface, with stable and unstable measured foliations $\mathscr{F}_{u}, \mathscr{F}_{s}$. Because of the results of $\S 1$, we are assuming that $f$ fixes each $s \in \operatorname{Sing}(f)$, and also fixes the separatrices at $s$.

An $\mathscr{F}_{u}, \mathscr{F}_{s}$ rectangle in $f$ is the image $R$ of an embedding $I \times I \rightarrow S$ such that horizontal segments go to leaf segments of $\mathscr{F}_{u}$, and vertical segments go to leaf segments of $\mathscr{F}_{s}$. We use $\partial_{s} R$ to denote the part of $\partial R$ lying on leaves of $\mathscr{F}_{s}$, and $\partial_{u} R$ to denote the part of $\partial R$ lying on leaves of $\mathscr{F}_{u} ; \partial_{u} R$ is thus the horizontal boundary' of $R$, and $\partial_{s} R$ is the 'vertical boundary' of $R$. In the literature, if $\sigma$ is a component of $\partial_{s} R$ or $\partial_{u} R$, points in int $(\sigma)$ can be either singularities or regular points; for our purposes, we shall assume that int $(\sigma)$ consists entirely of regular points. Corners of $R$ can be singularities, however.

A Markov partition for $f$ is a finite collection $\mathcal{M}$ of $\mathscr{F}_{u}, \mathscr{F}_{s}$ rectangles satisfying the following properties:

(i) $\mathcal{M}$ covers $S$;

(ii) two distinct rectangles in $\mathcal{M}$ have disjoint interiors;

(iii) for any $R, R^{\prime} \in \mathcal{M}, f($ int $(R)) \cap$ int $\left(R^{\prime}\right)$ is either empty or the interior of a single $\mathscr{F}_{u}, \mathscr{F}_{s}$ rectangle;

(iv) for any $R, R^{\prime} \in \mathcal{M}$ and any component $\sigma$ of $\partial_{u} R$, if $f^{-1}$ (int $\left.(R)\right) \cap$ int $\left(R^{\prime}\right)$ is the interior of a rectangle $R^{\prime \prime}$, and if $f^{-1}(\sigma) \cap R^{\prime \prime} \neq \varnothing$, then there is a component $\sigma^{\prime}$ of $\partial_{u} R^{\prime}$ such that $f^{-1}(\sigma) \subset \sigma^{\prime}$;

(v) for any $R, R^{\prime} \in \mathcal{M}$ and any component $\sigma$ of $\partial_{s} R$, if $f($ int $(R)) \cap$ int $\left(R^{\prime}\right)$ is the interior of a rectangle $R^{\prime \prime}$ and $f(\sigma) \subset R^{\prime \prime} \neq \varnothing$, then there is a component $\sigma^{\prime}$ of $\partial_{s} R^{\prime}$ such that $f(\sigma) \subset \sigma^{\prime}$.

In [FLP], it is shown that a Markov partition $\mu$ always exists for a pseudo-Anosov map $f$. In fact, the particular construction given in [FLP] has the following additional 
properties:

(vi) given an $n$-pronged singularity $s$ of $f, s$ is a corner of exactly $2 \cdot n$ rectangles in $\mathcal{M}$;

(vii) for any $R \in \mathcal{M}, \partial_{u} R$ consists of segments of singular separatrices of $\mathscr{F}_{u}$;

(viii) for any $R \in \mathcal{M}, \partial_{s} R$ consists of segments of singular separatrices of $\mathscr{F}_{s}$.

The construction is given in exposé $10, \S \mathrm{V}$, starting page 201 of [FLP], and refers back to exposé $9, \S \mathrm{V}$. The heart of the construction is given in lemme 9 , exposé 9 , $\S \mathrm{V}$ : one chooses a collection $\tau$ consisting of short initial segments of each unstable separatrix, with endpoints on stable separatrices, with the property that $f(\tau) \subset \tau$. The surface then naturally decomposes (lemma 11) into rectangles satisfying all properties but (iii). By choosing a subdivision, property (iii) is also obtained (exposé $10, \S \mathrm{V}$, pp. 201-202), retaining the rest of the properties.

Recall now how a subshift of finite type is constructed from a Markov partition. Define $A: \mathcal{M} \times \mathcal{M} \rightarrow\{0,1\}$ so that $A\left(R, R^{\prime}\right)=1$ if and only if $f(\operatorname{int}(R)) \cap$ int $\left(R^{\prime}\right) \neq \varnothing$; $A$ is called the transition matrix of $\mathcal{M}$, and it is a Perron-Froebenius matrix, i.e. there exists some $n \geq 1$ such that $A^{n}$ has all positive entries [FLP, exposé $10, \S \mathrm{VI}$, Lemma 1, p. 205]. Give $\mathcal{M}^{\mathbf{Z}}$ the product topology; following Bowen's convention, elements of $\mathcal{M}^{\mathbf{Z}}$ are denoted $\underline{m}$ with $\underline{m}=\left(m_{n}\right)_{n \in \mathbf{Z}}$, so each $m_{n}$ is an $\mathscr{F}_{u}, \mathscr{F}_{s}$ rectangle. Define the shift homeomorphism $\rho: \mathcal{M}^{\mathbb{Z}} \rightarrow \mathcal{M}^{\mathbb{Z}}$ by $\rho(\underline{m})=\underline{m}^{\prime}$, where $m_{n}^{\prime}=m_{n-1}$. Let $S_{A}=\left\{\underline{m} \in \mathcal{M}^{\mathbb{Z}} \mid A\left(m_{n}, m_{n+1}\right)=1\right.$ for all $\left.n \in \mathbb{Z}\right\}$ with the subspace topology; $S_{A}$ is invariant under $\rho$. In general, the term symbolic sequence will refer to an element of $S_{A}$, and the term finite symbolic sequence will refer to a finite segment of an element of $S_{A}$. Also, a symbolic loop of length $K$ is a circular sequence of the form $\underline{m}=\left(m_{k}\right)_{k \in Z / K}$, where $A\left(m_{k}, m_{k+1}\right)=1$ for each $k \in \mathbb{Z} / K$; such a sequence is sometimes written out as $\left(m_{1}, \ldots, m_{K}\right)$, where it is to be understood that $1, \ldots, K$ are considered as representatives of $\mathbb{Z} / K$; the sequence is also written out as $\left(m_{0}, m_{1}, \ldots, m_{K}=m_{0}\right)$. (Note: we shall not in general distinguish between a symbolic loop $\underline{m}=\left(m_{i}\right)_{i \in \mathbf{Z} / K}$ and any loop obtained by a circular order preserving permutation of the index set $\mathbb{Z} / K$.) Given a symbolic loop $\underline{m}=\left(m_{k}\right)_{k \in Z / K}$, there is an associated symbolic sequence $\tilde{m}=\left(\tilde{m}_{i}\right)_{i \in Z}$ where $\tilde{m}_{i}=m_{k}$ whenever $k$ is the congruence class of $i \bmod K$.

Define a map $q: S_{A} \rightarrow S$ as follows: given $\underline{m} \in S_{A}$, the sequence of sets $B_{N}(\underline{m})=$ $\bigcap\left\{f^{n}\left(m_{-n}\right) \mid-N \leq n \leq N\right\}$ is a nested sequence of $\mathscr{F}_{u}, \mathscr{F}_{s}$ rectangles whose intersection is a single point, which is defined to be $q(\underline{m})$. Then $q$ is a continuous semiconjugacy taking the shift map $\rho: S_{A} \rightarrow S_{A}$ to the pseudo-Anosov map $f: S \rightarrow S$, i.e. $q \circ \rho=f \circ q$. Note that the semi-conjugacy $q$ is finite-to-one. More precisely, given $x \in S$, the pre-images of $x$ under $q$ can be counted as follows. If $x$ is an $n$-pronged singularity, there are exactly $2 \cdot n$ pre-images; if $x$ is in the intersection of a stable singular separatrix and an unstable singular separatrix, there are exactly 4 preimages; if $x$ is any other point in a stable or unstable singular separatrix, there are exactly 2 pre-images; otherwise, there is exactly 1 pre-image.

Now we describe the symbolic dynamics of the suspension flow of a pseudoAnosov. Suppose we are given a 3-manifold $M$, a fibered face $\sigma$ of $B_{x}(M)$, and $\phi$ the associated pseudo-Anosov flow on $M$; once and for all, we fix a distinguished section $S$ of $\phi$, with pseudo-Anosov first return map $f$, and we assume that $f$ fixes 
all its singular separatrices. Let $\phi$ be parametrized so that the return time to $S$ is everywhere equal to 1 . Let $\mathcal{M}$ be a Markov partition for $f$, with transition matrix $A$. For each Markov rectangle $B \in \mathcal{M}$, let $B^{+}=B \cdot[0,1]$ be the corresponding flow box for $\phi$, and let $\mathcal{M}^{+}=\left\{B^{+} \mid B \in \mathcal{M}\right\}$.

The semi-conjugacy $q: S_{A} \rightarrow S$ induces a semi-conjugacy of flows, as follows. Let $T_{p}$ denote the mapping torus of $\rho$, i.e. the space $S_{A} \times \mathbb{R}$ modulo the identification of $\underline{m} \times t$ with $\rho(\underline{m}) \times(t-1)$, and let Susp $(\rho)$ denote the suspension flow of $\rho$ on $T_{\rho}$. Let $Q: T_{\rho} \rightarrow M$ be defined by $Q(\underline{m} \times t)=\phi(q(\underline{m}), t)$; this is easily checked to be well-defined. Evidently $Q$ is a semi-conjugacy from the flow $\operatorname{Susp}(\rho)$ to the flow $\phi$. Given $\underline{m} \in S(A)$, we shall use $O(\underline{m})$ to denote the orbit $Q(\underline{m} \times \mathbb{R})=Q(\underline{m}) \cdot \mathbb{P}$ of the point $Q(\underline{m})$ under the flow $\phi$. Notice that $\rho(\underline{m})$ is precisely the point $Q(\underline{m} \times 0)=$ $q(\underline{m})$.

By a periodic orbit of a flow we mean a map of $S^{1}$ into the space of the flow which locally immerses $S^{1}$ into flow segments and takes the local angular parameter on $S^{1}$ to the time parameter of a flow segment with constant derivative; a primitive periodic orbit is an embedding of $S^{1}$. Notice that a periodic orbit of a flow on a manifold $M$ determines a homology class in $H_{1}(M ; \mathbb{Z})$.

Given a symbolic loop $\underline{m}=\left(m_{k}\right)_{k \in Z / K}$, there is a corresponding periodic orbit of Susp ( $\rho$ ), whose image in $M$ is a periodic orbit of $\phi$ denoted $O(\underline{m})$; the corresponding homology class in $H_{1}(M ; \mathbb{Z})$ is denoted $[O(\underline{m})]$. A periodic orbit of $\phi$ can be either regular or singular, depending on whether or not the first return to a local crosssection looks like a regular point or a singular point of a pseudo-Anosov. A singular periodic orbit is $n$-pronged if the local first return map looks like an $n$-pronged singularity of a pseudo-Anosov. Given a periodic point $x$ of $f$ of period $k$, let Susp $(x)$ denote the corresponding primitive periodic orbit of $\phi$. If $x$ is a regular point of $f$, then Susp $(x)$ is regular. If $x$ is an $n$-pronged singularity of $f$, then Susp $(x)$ is an $n$-pronged singular orbit.

Notice that every regular periodic orbit of $\phi$ is equal to $O(\underline{m})$ for a unique symbolic loop $\underline{m}$ and $O(\underline{m})$ is primitive if and only if $\underline{m}$ is primitive. This follows from the fact that a regular periodic point $x$ of $f$ has a unique symbolic sequence, since $x$ does not lie on any stable or unstable singular separatrix. Similarly, an $n$-pronged singular periodic orbit of $\phi$ is equal to $O(\underline{m})$ for exactly $2 n$ distinct symbolic loops $\underline{m}$, and $O(\underline{m})$ is primitive if and only if $\underline{m}$ is primitive; in fact, each such $\underline{m}$ is a constant symbolic loop, and $O(\underline{m})$ is primitive if and only if $\underline{m}$ has length 1 . This follows from the fact that $f$ fixes all singular points and their separatrices, so each of the $2 n$ symbolic sequences of an $n$-pronged singularity $\boldsymbol{x}$ are constant. From these considerations, it also follows that for a general periodic point $x$ of $f$, if $\operatorname{susp}(x)=\mathrm{O}(\underline{m})$, and if $k$ is the least period of $x$, then $\underline{m}$ has length $k$.

\section{The chain kernel}

Now we turn to the proof proper of the $\mathbb{Z}$-Spectral Decomposition Theorem. Once and for all, fix an element $\alpha \in \partial($ Cone $(\sigma)) \cap H^{1}(M ; \mathbb{Z})$, and suppose that $\alpha$ is a primitive element of $H^{1}(M ; \mathbb{Z})$. 
First we consider the homology classes of $\varepsilon, T$ cycles of $\phi$, and their intersection numbers with $\alpha$. Given $\alpha \in H^{1}(M)$, and given an $\varepsilon, T$ cycle $X=$ $\left(\mathrm{x}_{0}, \ldots, \mathrm{x}_{\mathrm{n}} ; \mathrm{t}_{1}, \ldots, \mathrm{t}_{\mathrm{n}}\right)$, if $\varepsilon$ is smaller than the injectivity radius $\operatorname{inj}(M)$ of $M$, then there is a well-defined intersection number $\langle\alpha, X\rangle$. To define this number, first let $\bar{X}$ be a closed path in $M$ obtained by starting from $x_{0}$, proceeding along the flow line to $x_{0} \cdot t_{1}$, passing along a path to $x_{1}$ which stays completely in $B\left(x_{0} \cdot t_{1}, \varepsilon\right)$, proceeding along the flow line to $x_{1} \cdot t_{2}$, etc., eventually closing up at the point $x_{n}=x_{0}$. Since $\varepsilon<\operatorname{inj}(M)$, the closed path $\bar{X}$ is well-defined up to homotopy, independent of the choice, for each $i=1, \ldots, n$, of a small path from $x_{i-1} \cdot t_{i}$ to $x_{i}$. Thus, the intersection number $\langle\alpha, X\rangle=\langle\alpha, \bar{X}\rangle$ is well-defined.

The chain kernel of $\alpha$, denoted $R(\alpha)$, is defined to be the set of points $x$ such that for all $0<\varepsilon<\operatorname{inj}(M)$ and for all $T>0$, there exists an $\varepsilon, T$ cycle $X$ through $x$ such that $\langle\alpha, X\rangle=0$. For example, any periodic orbit of $\phi$ on which $\alpha$ vanishes algebraically is contained in the chain kernel of $\alpha$. It is a simple exercise to show that $R(\alpha)$ is a closed set invariant under $\phi$. Thus, if we define $L(\alpha)=\operatorname{cl}(\bigcup$ \{periodic orbits of $\phi$ on which $\alpha$ vanishes\}), then it follows that $L(\alpha) \subset R(\alpha)$.

Notice that $R(\alpha)$ depends only on the boundary face $F \subset$ Cone $(\sigma)$ such that $\alpha \in \operatorname{int}(F)$. In fact, if $G=F^{*}$ is the face of Cone $\left(D_{\phi}\right)$ which is dual to $F$, then $R(\alpha)=\{x \mid$ there exist $\varepsilon, T$ cycles $X$ through $x$ such that $[\bar{X}] \in G\}$. This follows directly from the easily established fact that $G=\operatorname{Ker}(\alpha) \cap \operatorname{Cone}\left(D_{\phi}\right)$. We shall occasionally adopt the notation $R(F)$ or $R(G)$ for the set $R(\alpha)$, when $\alpha \in \operatorname{int}(F)$ and $G=F^{*}$. Theorem 3.4 below, which gives Fried's characterization of $D_{\phi}$, shows that for each face $G$ of Cone $\left(D_{\phi}\right)$, there is always a periodic orbit $X$ of $\phi$ such that $[X] \in G$; from the comments of the previous paragraph, it follows that $R(G) \neq \phi$. This implies that the collection of all chain kernels for the flow $\phi$, which form a partially ordered set under inclusion, is isomorphic to the collection of faces of Cone $\left(D_{\phi}\right)$ partially ordered by inclusion, and is also isomorphic to the collection of faces of Cone $(\sigma)$ partially ordered by containment.

The chain kernel of $\alpha$ is the key to understanding how the qualitative dynamics of $\phi$ is related to the cohomology class $\alpha$. Our ultimate goal is to show that $R(\alpha)$ has finitely many chain components, each of which lifts homeomorphically to a chain component of $R(\tilde{\phi})$ (once a lift of a single point is chosen), and that the total lift of $R(\alpha)$ is $R(\tilde{\phi})$; this takes up most of $\S \S 3-7$, and properties (A) and (B) of the $\mathbb{Z}$-Spectral Decomposition Theorem will follow from such considerations. In $\S 7$, we also analyze the homology properties of orbits of $\phi$ which are not in $R(\alpha)$, in order to understand the transient behavior of the flow $\tilde{\phi}$, and prove properties (C) and (D). Using the groundwork of the previous sections, property (E) is proven in $\S 8$.

We shall begin the study of $R(\alpha)$ by analyzing the symbolic dynamics of the subset $L(\alpha)$. At this point, it is not clear whether or not $L(\alpha)$ is all of $R(\alpha)$, so for the moment we must be content with analyzing $L(\alpha)$; later we shall see how to extend this analysis to all of $R(\alpha)$. As a consequence of the symbolic dynamics analysis of $L(\alpha)$, we shall prove in Proposition 3.7 that $L(\alpha)$ has only finitely many chain components under the restricted flow $\phi \mid L(\alpha)$; this is a key step in the proof that $R(\alpha)$ has only finitely many chain components. 
In order to analyze $L(\alpha)$, we need a method for deciding which periodic orbits of $\phi$ are contained in $R(\alpha)$. Since periodic orbits are encoded as symbolic loops of $A$, what we want is a method for deciding which symbolic loops $\underline{m}$ satisfy the condition $\mathrm{O}(\underline{m}) \subset R(\alpha)$. First we need some combinatorial tools.

Given two symbolic loops $\underline{m}=\left(m_{i}\right)_{i \in \mathbf{Z} / L}$ and $\underline{m}^{\prime}=\left(m_{j}^{\prime}\right)_{j \in \mathbf{Z} / L}$, suppose that $m_{0}=m_{0}^{\prime}$. Define a new loop Splice $\left(\underline{m}, \underline{m}^{\prime}\right)=\underline{m}^{\prime \prime}$, where $\underline{m}^{\prime \prime}=\left(m_{i}^{\prime \prime}\right)_{i \in Z / L+L}$, and

$$
m_{i}^{\prime \prime}= \begin{cases}m_{i} & \text { if } 0 \leq i \leq L \\ m_{i-L}^{\prime} & \text { if } L \leq i \leq L+L^{\prime} .\end{cases}
$$

This is evidently a well-defined symbolic loop. It is not independent of cyclic permutation of indices, but there are two kinds of cyclic permutations which do not alter the spliced loop. When $\underline{m}$ (or $\underline{m}^{\prime}$ ) is not primitive, we can cyclically permute by a multiple of the primitive period. Also, when there are integers $P, Q \geq 0$ such that $P+Q<\min \left\{L, L^{\prime}\right\}$, and $m_{k}=m_{k}^{\prime}$ whenever $-P \leq k \leq Q$, then if we cyclically permute $\underline{m}$ and $\underline{m}^{\prime}$ by the same amount $k$ where $-P \leq k \leq Q$, the spliced loop does not change.

One important property of the splicing operation is that it behaves well when passing to homology classes. That is, when Splice $\left(\underline{m}, \underline{m}^{\prime}\right)$ is defined, we have the splice equation:

$$
\left[O\left(\text { Splice }\left(\underline{m}, \underline{m}^{\prime}\right)\right)\right]=[O(\underline{m})]+\left[O\left(\underline{m}^{\prime}\right)\right] \text { in } H_{1}(M ; \mathbb{Z}) \text {. }
$$

This is a standard fact in symbolic dynamics, and it is usually proven under the assumption that the Markov flow boxes $\left\{B^{+} \mid B \in \mathcal{M}\right\}$ have small diameter; this assumption can always be achieved by refining the Markov partition. However, there is an argument which uses only the fact that each local section $B$ is actually a topological rectangle $I \times I$, so $B^{+}$is a topological box. Letting $\gamma=\mathrm{O}(\underline{m}), \gamma^{\prime}=\mathrm{O}\left(\underline{m}^{\prime}\right)$, and $\gamma^{*}=\mathrm{O}\left(\right.$ Splice $\left.\left(\underline{m}, \underline{m}^{\prime}\right)\right)$, we shall explicitly construct a homology between the cycle $\gamma+\gamma^{\prime}$ and the cycle $\gamma^{*}$; the construction is illustrated in diagram 3. As $\gamma^{*}$ parallels $\gamma$ through the sequence of boxes $m_{1}^{+}, \ldots, m_{L-1}^{+}$, map the strip $I \times I$ into $M$ with $I \times 0$ mapping into $\gamma, I \times 1$ mapping into $\gamma^{*}, 0 \times I$ mapping into the top of $m_{0}^{+}$, and $1 \times I$ mapping into the bottom of $m_{0}^{+}$. Similarly, a strip can be constructed between $\gamma^{\prime}$ and $\gamma^{*}$ through the sequence of boxes $m_{1}^{\prime+}, \ldots, m_{L^{\prime}-1}^{\prime+}$. Finally, the four

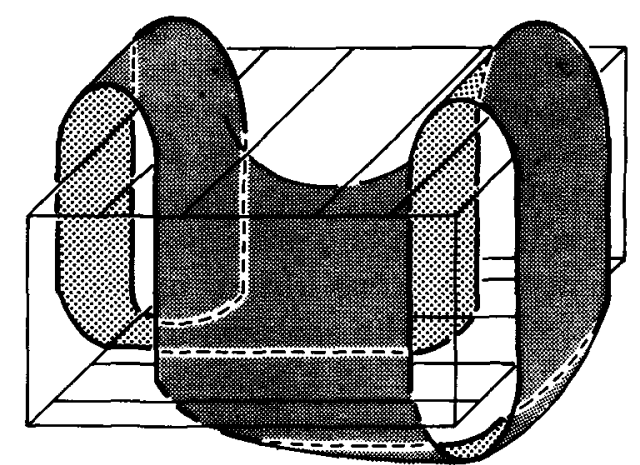

Diagram 3. A homology which proves the splice equation. 
bare boundary segments of these two strips, two on the top of $m_{0}^{+}$and two on the bottom of $m_{0}^{+}$, together with the four bare segments of $\gamma \cup \gamma^{\prime} \cup \gamma^{*}$ passing vertically through $m_{0}^{+}$, bound a saddle shaped surface in $m_{0}^{+}$. The gluing maps between the two strips and the saddle shaped surface can easily be shown to preserve orientation, and the resulting oriented immersed surface clearly has the cycle $\gamma+\gamma^{\prime}-\gamma^{*}$ as its boundary.

Let $\Gamma_{A}$ be the topological realization of $A: \Gamma_{A}$ is a directed graph (digraph) having vertex set $\mathcal{M}$, and with a directed edge $e\left(m, m^{\prime}\right)$ pointing from $m$ to $m^{\prime}$ if and only if $A\left(m, m^{\prime}\right)=1$. Since $A$ is Perron-Froebenius, it follows that $\Gamma_{A}$ is transitive, which means that for any two directed edges $e, e^{\prime}$ and any sufficiently large $n$, there is a directed path of length $n$ starting with $e$ and ending with $e^{\prime}$. We shall only make use of the weaker notion that $\Gamma_{A}$ is strongly connected, which means that for any two vertices $v, v^{\prime}$ in $\Gamma_{A}$, there is some directed path from $v$ to $v^{\prime}$, with no conditions on the length; equivalently, $\Gamma_{A}$ is connected and there is a directed loop through any directed edge of $\Gamma_{A}$.

For each symbolic loop $\underline{m}=\left(m_{i}\right)_{i \in \mathbf{Z} / L}$ of $A$, let $\gamma(\underline{m})$ denote the corresponding directed loop in $\Gamma_{A}$, based at the vertex $m_{0}$; this gives a 1-1 correspondence between symbolic loops of $A$ and based directed loops in $\Gamma_{A}$. When $\underline{m}=\left(m_{i}\right)_{i \in \mathbf{Z} / L}$ is altered by a cyclic permutation, then the base point of $\gamma(\underline{m})$ changes. Notice that when $\underline{m}$, $\underline{m}^{\prime}$ are two symbolic loops such that Splice $\left(\underline{m}, \underline{m}^{\prime}\right)$ is defined, then

$$
\gamma\left(\text { Splice }\left(\underline{m}, \underline{m}^{\prime}\right)\right)=\gamma(\underline{m}) * \gamma\left(\underline{m}^{\prime}\right),
$$

where $*$ indicates concatenation of based directed loops.

Now define a function $U_{\alpha}$ on directed loops of $\Gamma_{A}$ by $U_{\alpha}(\gamma(\underline{m}))=\langle\alpha, O(\underline{m})\rangle$. It follows from the splice equation $(*)$ and equation $(* *)$ that for any two based directed loops $\gamma, \gamma^{\prime}$ with the same base point, $U_{\alpha}\left(\gamma * \gamma^{\prime}\right)=U_{\alpha}(\gamma)+U_{\alpha}\left(\gamma^{\prime}\right)$. We wish to extend this function to a cohomology class on $\Gamma_{A}$, i.e. a function on all (unidirected) loops of $\Gamma_{A}$ which is additive under concatenation. To do this, we need:

Lемма 3.1. Directed loops span homology in strongly connected digraphs. Given a strongly connected digraph $G, H_{1}(G ; \mathbb{Z})$ is spanned by the homology classes of the directed loops.

Proof. Each element of $H_{1}(G ; \mathbb{Z})$ can be represented by a non-directed closed path $\gamma=e_{1}^{\alpha(1)} * e_{2}^{\alpha(2)} * \cdots * e_{K}^{\alpha(K)}$, where $\alpha(k)= \pm 1$ and $e_{i}$ represents a directed edge. We must show that $\gamma$ is homologous to a linear combination of directed closed paths. This is proven by induction on the number of edges that $\gamma$ traverses backwards, i.e. the size of the set $\{1 \leqslant k \leqslant K \mid \alpha(k)=-1\}$. We can assume that $\alpha(1)=-1$. Using strong connectivity, choose a directed path $\gamma^{\prime}$ from Head $\left(e_{1}\right)$ to Tail $\left(e_{1}\right)$. Notice that:

$$
\begin{aligned}
\operatorname{Head}\left(\gamma^{\prime}\right) & =\operatorname{Tail}\left(e_{1}\right)=\operatorname{Tail}\left(e_{2}^{\alpha(2)} * \cdots * e_{K}^{\alpha(K)}\right), \quad \text { and } \\
\text { Tail }\left(\gamma^{\prime}\right) & =\operatorname{Head}\left(e_{1}\right)=\operatorname{Head}\left(e_{2}^{\alpha(2)} * \cdots * e_{K}^{\alpha(K)}\right) .
\end{aligned}
$$

Thus, $\gamma_{1}=e_{1} * \gamma^{\prime}$ and $\gamma_{2}=e_{2}^{\alpha(2)} * \cdots * e_{K}^{\alpha(K)} * \gamma^{\prime}$ are closed loops in $\Gamma$, and we clearly have the following equation in $H_{1}(G ; \mathbb{Z}):[\gamma]=\left[\gamma_{2}\right]-\left[\gamma_{1}\right]$. Now $\gamma_{1}$ is directed,

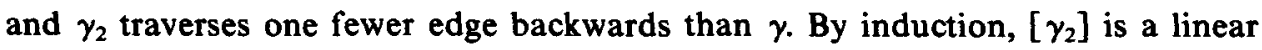
combination of classes of directed loops, and we are done. 
Now suppose one is given a function $U$ defined on the collection of directed loops, which is a homomorphism in the sense that if $\gamma, \gamma^{\prime}$ are directed closed loops with the same base point, then $U\left(\gamma * \gamma^{\prime}\right)=U(\gamma)+U\left(\gamma^{\prime}\right)$. Then $U$ can be extended to a homomorphism on the collection of all closed loops as follows: let $\gamma=$ $e_{1}^{\alpha(1)} * e_{2}^{\alpha(2)} * \cdots * e_{K}^{\alpha(K)}$ be as in the previous proof, where $\alpha(1)=-1$ and let $\gamma^{\prime}$ be a directed path from Head $\left(e_{1}\right)$ to Tail $\left(e_{1}\right)$; then define $U(\gamma)=U\left(\gamma_{2}\right)-U\left(\gamma_{1}\right)$, where $\gamma_{1}$ and $\gamma_{2}$ are defined as above. This is a definition by induction on the number of negative $\alpha(k)$. The fact that $U$ is a homomorphism on directed loops shows that $U(\gamma)$ is well-defined independent of the choice of $\gamma^{\prime}$. Thus, any homomorphism defined on the collection of directed loops of a strongly connected digraph $G$ can be extended to a cohomology class, i.e. an element of $H^{1}(G ; \mathbb{Z})$. In particular, $U_{\alpha}$ can be extended to a cohomology class on $H^{1}\left(\Gamma_{A} ; \mathbb{Z}\right)$, still denoted $U_{\alpha}$.

Given a cohomology class $U$ on a digraph $G, U$ is said to be positive if $U(\gamma)>0$ for every directed loop $\gamma$, and $U$ is non-negative if $U(\gamma) \geq 0$ for every directed loop $\gamma$. We shall use $C_{1}(G ; \mathbb{Z})$ to denote the cycle group freely generated by the collection of directed edges of $G$. A cocycle $u \in C^{1}(G ; \mathbb{Z})$ is called non-negative if $u(e) \geq 0$ for every directed edge $e$ of $G$.

Proposition 3.2. Existence of non-negative cocycles. Given a strongly connected digraph $G$ and a non-negative cohomology class $U$ on $G$, there exists a non-negative cocycle $u$ on $G$ representing the class $U$.

Proof. We shall reduce to the case where $U$ is positive, and then quote a result from the companion paper [M] to handle that case.

Suppose that $U$ is non-negative but not positive, and let $G_{U}$ be the union of all directed edges $e$ of $\Gamma$ such that for some directed loop $\gamma$ through $e, U(\gamma)=0$; in other words, $G_{U}$ is the union of all directed loops $\gamma$ such that $U(\gamma)=0$. It follows that each component of $G_{U}$ is strongly connected. Now we need:

LEMMA 3.3. For any non-directed loop $\gamma$ in $G_{U}, U(\gamma)=0$.

Proof. By applying Lemma 3.1 Directed Loops Span Homology in Strongly Connected Digraphs, we need only prove the lemma for a directed loop $\gamma$. In other words, not only does $G_{U}$ contain all directed loops $g$ such that $U(g)=0$, but $G_{U}$ contains no other directed loops.

Fix a directed loop $\gamma=e_{1} * \cdots * e_{K}$. By definition of $G_{U}$, for each $i=1, \ldots, K$ there is a directed loop $\gamma_{i}$ of the form $\gamma_{i}=e_{i} * d_{i}$, where $d_{i}$ is some directed path, and $U\left(\gamma_{i}\right)=0$. For each $i=1, \ldots, K$, define a directed loop $\alpha_{i}=$ $e_{1} * e_{2} * \cdots e_{i} * d_{i} * \cdots * d_{2} * d_{1}$. To check that $\alpha_{i}$ is actually a loop, the only non-obvious thing to check is that Head $\left(d_{k}\right)=$ Tail $\left(d_{k-1}\right)$ for each $k=1, \ldots, K$; this follows because Head $\left(d_{k}\right)=\operatorname{Tail}\left(e_{k}\right)=\operatorname{Head}\left(e_{k-1}\right)=\operatorname{Tail}\left(d_{k-1}\right)$. Notice that by changing the base points of $\alpha_{i}$ and $\alpha_{i+1}$, we have $\alpha_{i+1}=\alpha_{i} * \gamma_{i}$. Thus, we have $U\left(\alpha_{i+1}\right)=U\left(\alpha_{i}\right)+U\left(\gamma_{i}\right)$, and it follows by induction that $U\left(\alpha_{K}\right)=0$. Also, 
$d_{K} * \cdots * d_{1}$ is a loop, and we have $\alpha_{K}=\gamma *\left(d_{K} * \cdots * d_{1}\right)$, so

$$
U(\gamma)+U\left(d_{K} * \cdots * d_{1}\right)=U\left(\alpha_{K}\right)=0 .
$$

Since $U$ is non-negative, both terms on the left hand side of this equation are non-negative, and their sum is zero, so it follows that they are both zero. In particular, $U(\gamma)=0$.

Returning to the discussion previous to the lemma, define a quotient map $q: G \rightarrow G^{\prime}$ to a directed graph $G^{\prime}$, by collapsing each component of $G_{U}$ to a single vertex. Thus, $G^{\prime}$ has one directed edge for every directed edge in $G-G_{U}$. Notice that $G^{\prime}$ is strongly connected, for if $e^{\prime}$ is a directed edge of $G^{\prime}$, then considered as a directed edge of $G$, there is a directed loop $\gamma$ in $G$ passing through $e^{\prime}$, so $q(\gamma)$ is a directed loop in $G^{\prime}$ passing through $e^{\prime}$.

Define a cohomology class $U^{\prime} \in H^{\prime}\left(G^{\prime} ; \mathbb{Z}\right)$ as follows: for each non-directed loop $\gamma^{\prime}$ of $G^{\prime}$, choose a non-directed loop $\gamma$ of $G$ projecting to $\gamma^{\prime}$ under the quotient map $q$, and define $U^{\prime}\left(\gamma^{\prime}\right)=U(\gamma)$. To see that $U^{\prime}$ is well-defined, notice that if $\delta$ is another directed loop with $q(\delta)=\gamma$, then the homology class $[\gamma]-[\delta]$ is a sum of classes of non-directed loops contained entirely in $G_{U}$. As a direct consequence of Lemma 3.3, $U([\gamma]-[\delta])=0$, so $U^{\prime}$ is well-defined.

We claim that $U^{\prime}$ is positive. For let $\gamma^{\prime}$ be a non-trivial directed loop of $G^{\prime}$. Since each component of $G_{U}$ is strongly connected, there exists a directed loop $\gamma$ in $G$ such that $q(\gamma)=\gamma^{\prime}$. Moreover, $\gamma \not \subset G_{U}$, since $\gamma^{\prime}$ is non-trivial. Thus, as a consequence of Lemma 3.3, $U^{\prime}\left(\gamma^{\prime}\right)=U(\gamma)>0$.

Now we invoke the proposition Existence of positive cocycles from the companion paper [M], which says that since $G^{\prime}$ is a strongly connected digraph and $U^{\prime}$ is a positive cohomology class on $G^{\prime}$, then $U^{\prime}$ is represented by a non-negative cocycle $u^{\prime}$ of $G^{\prime}$. Now define a cocycle $u=q^{*}\left(u^{\prime}\right)$ on $G$. Evidently $u$ represents $U$. Also, $u$ is non-negative, having non-negative value on edges of $G-G_{U}$, and zero on edges of $G_{U}$.

Returning to the main track of our discussion, where $U_{\alpha}$ is a non-negative cohomology class on the strongly connected digraph $\Gamma_{A}$, it follows that there exists a non-negative cocycle $u_{\alpha}$ on $\Gamma_{A}$ representing $U_{\alpha}$. As a shorthand, when $A\left(m, m^{\prime}\right)=1$ we shall write $u_{\alpha}\left(m, m^{\prime}\right)$ for $u_{\alpha}\left(e\left(m, m^{\prime}\right)\right)$; and for a symbolic loop $\underline{m}=\left(m_{i}\right)_{i \in \mathbf{Z} / K}$ we shall write $u_{\alpha}(\underline{m})$ for $u_{\alpha}(\gamma(\underline{m}))=\sum_{i \in \mathbf{Z} / K} u_{\alpha}\left(m_{i}, m_{i+1}\right)$.

Now we can describe explicitly what subset of Cone $\left(D_{\phi}\right)$ is represented by homology classes of periodic orbits of $\phi$. Define the homology kernel of $\alpha$ to be $\operatorname{Ker}(\alpha)=\left\{c \in H_{1}(M ; \mathbb{R}) \mid\langle\alpha, c\rangle=0\right\}$, and define the kernel of homology directions to be $\operatorname{Ker}_{\phi}(\alpha)=\operatorname{Ker}(\alpha) \cap \operatorname{Cone}\left(D_{\phi}\right)$. From the Fried duality property for pseudoAnosov flows it follows that, if $F$ is the face of Cone $(\sigma)$ such that $\alpha \in$ int $(F)$, then $\operatorname{Ker}_{\phi}(\alpha)=F^{*}$.

We shall need another result of Fried, from [F2, Lemma 3, p. 361]; see also [F1, theorem 6, p. 260]:

THEOREM 3.4. (Fried). Cone $\left(D_{\phi}\right)$ is the smallest convex cone in $H_{1}(M ; \mathbb{R})$ containing all homology classes of the form $[\mathrm{O}(\underline{m})]$ where $\underline{m}$ is a simple symbolic loop of $A$. 
Let $M(\alpha)$ denote the collection of all simple symbolic loops $\underline{m}$ such that $U_{\alpha}(\underline{m})=$ 0 . As an immediate corollary, we have:

COROLlary 3.5. $\operatorname{Ker}_{\phi}(\alpha)$ is the smallest convex cone in $H_{1}(M ; \mathbb{Z})$ containing all homology classes of the form $[\mathrm{O}(\underline{m})]$ where $\underline{m} \in M(\alpha)$.

Now let $A(\alpha): \mathcal{M} \times \mathcal{M}$ denote the sub-matrix of $A$ defined by $A(\alpha)\left(m, m^{\prime}\right)=1$ if and only if $\left(m, m^{\prime}\right)$ occurs as a transition among the loops in the collection $M(\alpha)$. Equivalently, $\Gamma_{A(\alpha)} \subset \Gamma_{A}$ is the union of all simple directed loops on which $U_{\alpha}$ vanishes. We wish to show that $\Gamma_{A(\alpha)}$ is, in fact, the union of all directed loops on which $U_{\alpha}$ vanishes.

Given collections $M, M^{\prime}$ of symbolic loops which are closed under cyclic permutation of indices, let $M * M^{\prime}$ denote the collection of all loops of the form Splice $\left(\underline{m}, \underline{m}^{\prime}\right)$, where $\underline{m} \in M, \underline{m}^{\prime} \in M^{\prime}$, and $m_{0}=m_{0}^{\prime}$. Let $M^{k}$ be defined inductively as $M^{k-1} * M$, and let $M^{*}$ be defined as $\bigcup\left\{M^{k} \mid k \in \mathbb{Z}_{+}\right\}$.

Notice that $M(\alpha)^{*}=\left\{\right.$ symbolic loops $\left.\underline{m} \mid U_{\alpha}(\underline{m})=0\right\}$. The inclusion $\subset$ is clear. To get the opposite inclusion, suppose that $U_{\alpha}(\underline{m})=0$. If $\underline{m}$ is not already simple, then after cyclically permuting indices, there are shorter symbolic loops $\underline{m}^{\prime}$ and $\underline{m}^{\prime \prime}$ such that $\underline{m}=$ Splice $\left(\underline{m}^{\prime}, \underline{m}^{\prime \prime}\right)$, and from the splice equation it follows that

$$
U_{\alpha}\left(\underline{m}^{\prime}\right)+U_{\alpha}\left(\underline{m}^{\prime \prime}\right)=U_{\alpha}(\underline{m})=0 .
$$

Both terms on the left hand side of the equation are non-negative, and their sum is zero, therefore they must both be zero. By induction, it follows that $\underline{m}^{\prime}, m^{\prime \prime} \in M(\alpha)^{*}$, so $\underline{m} \in \mathbf{M}(\alpha)^{*}$.

It is evident that $\Gamma_{A(\alpha)}$ is the union of all symbolic loops $\underline{m}$ in $M(\alpha)^{*}$, so we have:

Proposition 3.6. Characterization of $A(\alpha) . \Gamma_{A(\alpha)}=\bigcup\left\{\gamma(\underline{m}) \mid U_{\alpha}(\gamma(\underline{m}))=0\right\}$.

Now we can use this result to analyze the symbolic dynamics of the invariant set $L(\alpha)=\operatorname{cl}(\bigcup$ periodic orbits of $\phi$ on which $\alpha$ vanishes\}), which is a subset of $R(\alpha)$. It is evident from Proposition 3.6 that $S_{A(\alpha)}$ is the closure in $S_{A}$ of $\bigcup\{\underline{\tilde{m}} \mid \underline{m}$ is periodic and $U_{\alpha}(\underline{m})=0$ \}. Let $T_{A(\alpha)} \subset T_{\rho}$ denote the image of $S_{A(\alpha)} \times \mathbb{R}$ under the quotient $S_{A} \times \mathbb{R} \rightarrow T_{\rho}$. Clearly Susp $(\rho) \mid T_{A(\alpha)}$ is the same as Susp $\left(\rho \mid S_{A(\alpha)}\right)$. It is also clear that under the semi-conjugacy $Q: T_{\rho} \rightarrow M, T_{A(\alpha)}$ is the pre-image of $L(\alpha)$.

It is a standard fact in the theory of symbolic flows that, since every component of $A(\alpha)$ is strongly connected, then $\operatorname{Susp}(\rho) \mid T_{A(\alpha)}=\operatorname{Susp}\left(\rho \mid S_{A(\alpha)}\right)$ is a chain recurrent flow, and the chain components of $\operatorname{Susp}(\rho) \mid T_{A(\alpha)}$ are in 1-1 correspondence with the components of the directed graph $A(\alpha)$. Thus, $\operatorname{Susp}(\rho) \mid T_{A(\alpha)}$ has finitely many chain components. Since $Q$ restricts to a semi-conjugacy from Susp $(\rho) \mid T_{A(\alpha)}$ onto $\phi \mid L(\alpha)$, each chain component of $L(\alpha)$ contains the image of a chain component of $T_{A(a)}$, so we have proven the main result of this section:

Proposition 3.7. $L(\alpha)$ has only finitely many chain components under the restricted flow $\phi \mid L(\alpha)$.

It would, of course, be very nice if $L(\alpha)$ were all of $R(\alpha)$. If we were working with a basic set of an Axiom $A$ flow, this would be true. However, for pseudo-Anosov 
flows this is in general false, because of the failure of shadowing. We now proceed to describe a larger portion of $R(\alpha)$.

Let $\mathscr{S}=\left(\mathscr{S}_{j}\right)_{j \in \mathbf{Z} / J}$, be a circular sequence of non-periodic orbits of the flow $\phi$ with the following property: For each $j \in \mathbb{Z} / J$, there exists a singular periodic orbit $\overline{\mathscr{S}}_{j}$ of $\phi$ with $L_{-}\left(\mathscr{S}_{j}\right)=L_{+}\left(\mathscr{S}_{j-1}\right)=\overline{\mathscr{S}}_{j}$; see $\S 0$ for the definitions of $L_{-}$and $L_{+}$. Then we say that $\mathscr{S}$ is a periodic quasi-orbit of $\phi$ (since we shall have little occasion to discuss 'non-periodic' quasi-orbits, we shall usually drop the adjective 'periodic').

Certain quasi-orbits $\mathscr{P}$ are 'non-shadowed' in the following sense: for each $j \in \mathbb{Z} / J$, let $L_{s}$ be the component of the local stable manifold $M_{s}$ of $\overline{\mathscr{S}}_{j}$ such that as $t \rightarrow+\infty$, $\mathscr{S}_{j-1}$ spirals into $\overline{\mathscr{Y}}_{j}$ along $L_{s}$; and let $L_{u}$ be the component of the unstable manifold $M_{u}$ of $\overline{\mathscr{S}}_{j}$ that as $t \rightarrow-\infty, \mathscr{S}_{j}$ spirals into $\overline{\mathscr{S}}_{j}$ along $L_{u}$. The collection of components of $M_{s}$ and $M_{u}$ is circularly ordered, and when $L_{s}$ and $L_{u}$ are non-adjacent in this circular ordering, then $\mathscr{S}$ is non-shadowed.

To explain the non-shadowed property more fully, let $D$ be a small transverse disc to the flow which is a local section near $\overline{\mathscr{S}}_{j}$. Since $\overline{\mathscr{P}}_{j}$ is singular, the first return map to $D$ looks locally like a pseudo-Anosov map near an $n$-pronged singularity for some $n \geq 3$, so $D$ is 'bifoliated' by stable and unstable foliations. Note that $M_{s} \cap D$ and $M_{u} \cap D$ consist respectively of $n$ stable and unstable 'separatrices' emerging from the point $\overline{\mathscr{Y}}_{j} \cap D$, and the unstable separatrices are interleaved with the stable separatrices. Now the first return map to $D$ fixes all separatrices (by hypothesis on $f$ ), so $\ell_{u}=L_{u} \cap D$ consists of an initial segment of an unstable separatrix; similarly for $\ell_{s}=L_{s} \cap D$. To say that $L_{s}$ and $L_{u}$ are not adjacent means that there is no bi-foliated rectangle $B$ in $D$ with $\ell_{s}, \ell_{u} \subset \partial B$.

The reason for introducing quasi-orbits is that we can repair the failure of shadowing arguments for pseudo-Anosov flows by augmenting the collection of closed orbits with the collection of non-shadowed quasi-orbits. As we shall see, we can also define what it means for a quasi-orbit to have zero intersection number with $\alpha$. In $\S 5$ we shall show, using a modified shadowing argument, that $R(\alpha)$ is the closure of the collection of periodic orbits and quasi-orbits having zero intersection number with $\alpha$.

Consider a quasi-orbit $\mathscr{S}=\left(\mathscr{S}_{j}\right)_{j \in Z / J}$; we define an intersection number $\langle\alpha, \mathscr{S}\rangle \in$ $\mathbb{Z}_{\geq} \cup\{+\infty\}$. If there exists $j \in \mathbb{Z} / J$ such that $\overline{\mathscr{S}}_{j} \not \subset L(\alpha)$, define $\langle\alpha, \mathscr{P}\rangle=\infty$. Otherwise, assuming that $\overline{\mathscr{S}}_{j} \subset L(\alpha)$ for all $j \in \mathbb{Z} / J$, we define a finite intersection number $\langle\alpha, \mathscr{P}\rangle \in \mathbb{Z}_{z}$. Note that $\left[\overline{\mathscr{P}}_{j}\right] \in \operatorname{Ker}_{\phi}(\alpha) \subset \operatorname{Ker}(\alpha)$, so it follows that $\mathscr{S}$ determines a well-defined element $\left[\mathscr{P}\right.$ ] of the group $H_{1}(M ; \mathbb{R}) / \operatorname{Ker}(\alpha)$. A closed path $Z$ representing $[\mathscr{P}]$ can be constructed by starting on $\overline{\mathscr{S}}_{1}$, jumping a small distance to $\mathscr{S}_{1}$, travelling along $\mathscr{S}_{1}$ and then jumping a small distance to $\overline{\mathscr{S}}_{2}$, then jumping a small distance to $\mathscr{S}_{2}$, travelling along $\mathscr{S}_{2}$ and then jumping a small distance to $\overline{\mathscr{S}}_{3}$, etc. Intersection number with $\alpha$ gives a well defined surjective homomorphism $H_{1}(M, \mathbb{R}) / \operatorname{Ker}(\alpha) \rightarrow \mathbb{R}$, so defined $\langle\alpha, \mathscr{S}\rangle$ to be the image under this homomorphism of $[\mathscr{S}]$. If $\langle\alpha, \mathscr{S}\rangle=0$, then it is clear that the $\mathscr{S} \subset R(\alpha)$ (we abuse notation by confusing $\mathscr{S}$ with the set $\left.\bigcup\left\{\mathscr{S}_{j} \mid j \in \mathbb{Z} / J\right\}\right)$.

Now let $L^{q}(\alpha)=L(\alpha) \cup \operatorname{cl}(\bigcup\{\mathscr{S} \mid \mathscr{S}$ is a periodic quasi-orbit, and $\langle\alpha, \mathscr{S}\rangle=0\})$; the superscript ' $q$ ' in the notation $L^{q}(\alpha)$ refers to 'quasi-orbits'. The main theorem 
on the structure of $R(\alpha)$ is:

TheOREM 3.8. Characterization of the Chain Kernel of $\alpha . R(\alpha)=L^{q}(\alpha)$.

It is clear that $L^{q}(\alpha) \subset R(\alpha)$. In $\S 5$, we shall prove the opposite inclusion, using a modified shadowing argument. This will be based on an analysis of the symbolic dynamics of quasi-orbits and of the set $L^{q}(\alpha)$ which is carried out in $\S 4$, generalizing the analysis of the symbolic dynamics of periodic orbits and of the set $L(\alpha)$. We shall also use the symbolic dynamics of quasi-orbits to prove in $\S 4$ that $L^{q}(\alpha)$ has only finitely many chain components; together with the above theorem, this shows that $R(\alpha)$ has only finitely many chain components. The symbolic dynamics of $L^{q}(\alpha)$ will also be useful in establishing homology properties of orbits of $\phi$ in $M-R(\alpha)$, which will be used in $\S 7$ to understand properties of transient orbits of the flow $\tilde{\phi}$.

\section{Symbolic dynamics of quasi-orbits}

Our next task is to develop a symbolic dynamics interpretation of a periodic quasi-orbit $\mathscr{S}$ of $\phi$, and use it to give a method for computing $\langle\alpha, \mathscr{Y}\rangle$. This will be applied to give a symbolic dynamics interpretation of $L^{q}(\alpha)$. It will follow immediately (Proposition 4.7) that $L^{q}(\alpha)$ has finitely many chain components.

We start with a lemma about symbolic loops of singular periodic orbits:

LEMMA 4.1. Let $\underline{m}$ be a primitive symbolic loop of $A$, and suppose that $\mathrm{O}(\underline{m})$ is a singular periodic orbit of $\phi$. Then $\underline{m}$ has length 1 . Thus, if $\underline{m}, \underline{m}^{\prime}$ are primitive symbolic loops such that both $\mathrm{O}(\underline{m}), \mathrm{O}\left(\underline{m}^{\prime}\right)$ are singular periodic orbits of $\phi$, then $\underline{m}$ and $\underline{m}^{\prime}$ are either identical or disjoint.

Proof. All but the last sentence was proven at the end of $\S 2$. The last sentence is a trivial consequence of the preceding.

Consider now a doubly indexed symbolic sequence of the form $\underline{m}=$ $\left(m_{i}^{j} \mid j \in \mathbb{Z} / J, i \in \mathbb{Z}\right)$, where $\underline{m}^{j} \in S_{A}$ for each $j \in \mathbb{Z} / J$, and $\underline{m}^{j}$ is non-periodic; each symbolic sequence $\underline{m}^{j}=\left(m_{i}^{j}\right)_{i \in Z}$ is called a piece of $\underline{m}$, so there are $J$ pieces altogether. Let $\mathscr{S}^{j}=O\left(\underline{m}^{j}\right)$, and suppose that $\mathscr{S}=\left(\mathscr{S}^{j}\right)$ is a periodic quasi-orbit. Then we say that $\underline{m}$ is a symbolic quasi-loop, and we write $\mathrm{O}(\underline{m})=\mathscr{S}$. From the previous lemma it follows that for each $j, \underline{m}^{j}=\left(m_{i}^{j}\right)_{i \in Z}$ is eventually constant as $i \rightarrow+\infty$; if $I$ is chosen minimally so that $\left(m_{i}^{j}\right)_{i \geq I}$ is constant, then we refer to $\left(m_{i}^{j}\right)_{i \geq I}$ as the positive tail of $\underline{m}^{j}$, and we write $m_{+}^{j}$ for the primitive period loop, which has length 1 . Also, since $\underline{m}^{j}$ is eventually constant as $i \rightarrow-\infty$, we can similarly define the negative tail of $\underline{m}^{j}$, and the primitive period loop $\underline{m}_{-}^{j}$ of length 1 . These loops have the property that $\mathrm{O}\left(\underline{m}_{+}^{j}\right)=\overline{\mathscr{S}}^{j}$ and $\mathrm{O}\left(\underline{m}_{-}^{j}\right)=\overline{\mathscr{S}}^{j-1}$.

A cyclic permutation of indices of a symbolic quasi-loop $\underline{m}=\left(j_{i}^{j} \mid j \in \mathbb{Z} / J, i \in \mathbb{Z}\right)$ is defined to be any combination of the following two operations: a cyclic permutation of the $j$ index; or for some fixed $j$, an order preserving permutation of the indices of $\underline{m}^{j}=\left(m_{i}^{i} \mid i \in \mathbb{Z}\right)$.

A symbolic quasi-loop $\underline{m}$ is simple if, whenever $m_{i}^{j}=m_{i}^{j^{\prime}}$, one of the following two possibilities holds: either $j=j^{\prime}$ and $m_{i}^{j}, m_{i}^{j}$ are in the same periodic tail of $\underline{m}^{j}$; 
or $j^{\prime}=j+1 \bmod J, m_{i}^{j}$ is in the positive tail of $\underline{m}^{j}$, and $m_{i^{\prime}}^{j^{\prime}}$ is in the negative tail of $\underline{m}^{j^{\prime}}$. In the latter case, note that $\underline{m}_{+}^{j}$ and $\underline{m}_{-}^{j^{\prime}}$ are the same symbolic loop. One special case to take note of is when $\underline{m}$ consists of single piece $\underline{m}^{0}$, i.e. when $J=1$ : the definition of simple then allows the possibility that $\underline{m}_{-}^{0}=\underline{m}_{+}^{0}$, as long as no symbol outside of the positive and negative periodic tail is repeated.

Splicing of symbolic quasi-loops with each other and with symbolic loops is defined analogously to splicing of symbolic loops. Given a symbolic quasi-loop $\underline{m}=\left(m_{i}^{j} \mid j \in \mathbb{Z} / J, i \in \mathbb{Z}\right)$, the 'splicing site' is defined to be $i=j=0$; the splicing site of a symbolic loop $\underline{m}^{\prime}=\left(m_{i}^{\prime}\right)_{i \in \mathbb{Z} / I}$ is defined to be $i=0$. Given symbolic loops or quasi-loops $\underline{m}, \underline{m}^{\prime}$, when the same symbols are located at the splicing sites, then Splice $\left(\underline{m}, \underline{m}^{\prime}\right)$ is defined in the obvious way.

There is another form of splicing two symbolic quasi-loops, called singular spacing, which we shall need. Let $\underline{m}=\left(\underline{m}^{i}\right)_{i \in \mathbf{Z} / l}, \underline{m}^{\prime}=\left(\underline{m}^{j}\right)_{j \in \mathbf{Z} / J}$ be symbolic quasi-loops, and suppose that $\mathrm{O}\left(\underline{m}_{+}^{I-1}\right)=\mathrm{O}\left(\underline{m}_{-}^{\prime 0}\right)$. Let $K=I+J$, and define Splices $\left(\underline{m}, \underline{m}^{\prime}\right)$ to be the symbolic quasi-loop $\underline{m}^{\prime \prime}=\left(\underline{m}^{\prime \prime k}\right)_{k \in \mathbb{Z} / K}$ with $\underline{m}^{\prime \prime k}=\underline{m}^{k}$ for $0 \leq k<I$, and $\underline{m}^{\prime \prime k}=$ $\underline{m}^{\prime k-I}$ for $I \leq k<I+J$.

Given two symbolic loops or quasi-loops $\underline{m}, \underline{m}^{\prime}$, we leave it to the reader to prove the generalized splice equation

$$
\left\langle\alpha, \mathrm{O}\left(\text { Splice }\left(\underline{m}, \underline{m}^{\prime}\right)\right)\right\rangle=\langle\alpha, \mathrm{O}(\underline{m})\rangle+\left\langle\alpha, \mathrm{O}\left(\underline{m}^{\prime}\right)\right\rangle
$$

whenever each term is defined; the terms in this equation all have values in $\mathbb{Z}_{+} \cup\{+\infty\}$, and equations involving $+\infty$ are interpreted in the obvious way.

Given a symbolic quasi-loop $\underline{m}=\left(m_{i}^{j} \mid j \in \mathbb{Z} / J, i \in \mathbb{Z}\right)$, we would like to be able to compute the value of $\langle\alpha, \mathrm{O}(\underline{m})\rangle$ exactly as before, using the cocycle $u_{\alpha} \in C^{1}\left(\Gamma_{A} ; \mathbb{Z}\right)$ representing $U_{\alpha} \in H^{1}\left(\Gamma_{A} ; \mathbb{Z}\right)$. That is, define $u_{\alpha}(\underline{m})=\sum u_{\alpha}\left(m_{i}^{j}, m_{i+1}^{j}\right)$, summed over all $j \in \mathbb{Z} / J$ and $i \in \mathbb{Z}$. This is an infinite sum of non-negative integers, and so has a value in $\mathbb{Z}_{\geq} \cup\{+\infty\}$. Unfortunately, it might not be true in general that $\langle\alpha, \mathrm{O}(\underline{m})\rangle=$ $u_{\alpha}(\underline{m})$; intuitively, what goes wrong is that $u_{\alpha}$ does not 'know' that different symbolic loops can represent the same singular orbit of $\phi$. However, this defect can be remedied:

LEMMA 4.2. The non-negative cocycle $u_{\alpha}$ can be chosen in its cohomology class so that for each symbolic loop or quasi-loop $\underline{m},\langle\alpha, O(\underline{m})\rangle=u_{\alpha}(\underline{m})$.

Proof. Define a directed graph $\Gamma_{A}^{\prime}$ as a quotient of $\Gamma_{A}$, by identifying directed loops $\gamma(\underline{m})$ and $\gamma\left(\underline{m}^{\prime}\right)$ whenever $\underline{m}, \underline{m}^{\prime}$ are symbolic loops of length 1 with $\mathrm{O}(\underline{m}), \mathrm{O}\left(\underline{m}^{\prime}\right)$ both equal to the same singular orbit of $\phi$ on which $\alpha$ vanishes. Thus, for certain $s \in \operatorname{Sing}(f, h), 2 n$ nodes of $\Gamma_{A}$ are identified, and $2 n$ directed loops of length 1 are identified. It is evident that $\Gamma_{A}^{\prime}$ is strongly connected. Note that each directed loop of $\Gamma_{A}^{\prime}$ corresponds uniquely to a symbolic quasi-loop $\underline{m}$ of $\Gamma_{A}$ such that $0 \leq U_{\alpha}(\underline{m})<$ $+\infty$; this allows us to define a $\mathbb{Z}_{\geq}$valued function $U_{\alpha}^{\prime}$ on directed loops of $\Gamma_{A}^{\prime}$ so that $U_{\alpha}^{\prime}$ pulls back to $U_{\alpha}$ under the quotient map $\Gamma_{A} \rightarrow \Gamma_{A}^{\prime}$. The generalized splice equation shows that $U_{\alpha}^{\prime}$ is a homomorphism on the directed loops of $\Gamma_{A}^{\prime}$, and so extends to a cohomology class in $H^{1}\left(\Gamma_{A}^{\prime} ; \mathbb{Z}\right)$. Evidently $U_{\alpha}^{\prime}$ is non-negative, so there is a non-negative cocycle $u_{\alpha}^{\prime}$ on $\Gamma_{A}^{\prime}$ representing $U_{\alpha}^{\prime}$. Taking $u_{\alpha}$ to be the pull-back of $u_{\alpha}^{\prime}$ under $\Gamma_{A} \rightarrow \Gamma_{A}^{\prime}$, the lemma follows. 
When $M, N$ denote collections of symbolic loops or quasi-loops which are invariant under cyclic permutation of indices, we shall use $M * N$ to denote all possible loops of the form Splice $(\underline{m}, \underline{n})$ or $\operatorname{Splice}^{s}(\underline{m}, \underline{n})$ with $\underline{m} \in M$ and $\underline{n} \in N$. $M^{k}$ and $M^{*}$ are similarly defined using splicing of loops and quasi-loops.

Let $M^{\prime}(\alpha)$ denote the union of $M(\alpha)$ with the collection of simple symbolic quasi-loops $\underline{m}$ such that $u_{\alpha}(\underline{m})=0$. Let $A^{\prime}(\alpha): \mathcal{M} \times \mathcal{M} \rightarrow\{0,1\}$ be the submatrix of $A$ such that $A^{\prime}(\alpha)\left(m, m^{\prime}\right)=1$ precisely when $\left(m, m^{\prime}\right)$ occurs as a transition among the elements of $M^{\prime}(\alpha)$; equivalently, $\Gamma_{A^{\prime}(\alpha)}$ is the subset of $\Gamma_{A}$ consisting of the union of all directed loops of the form $\gamma(\underline{m})$, where $\underline{m}$ is a simple symbolic loop satisfying $u_{\alpha}(\underline{m})=0$, or $\underline{m}=\underline{n}^{j}$ is a piece of a simple symbolic quasi-loop $\underline{n}$ satisfying $u_{\alpha}(\underline{n})=0$. It is not necessarily true that $\Gamma_{A^{\prime}(\alpha)}$ is strongly connected. However, we do have the following analogue of Proposition 3.6:

Proposition 4.3. Characterization of $A^{\prime}(\alpha) . \Gamma_{A^{\prime}(\alpha)}=\bigcup\{\gamma(\underline{m}) \mid \underline{m}$ is a symbolic loop satisfying $u_{\alpha}(\underline{m})=0$, or $\underline{m}=\underline{n}^{j}$ is a piece of a symbolic quasi-loop $\underline{n}$ satisfying $\left.u_{\alpha}(\underline{n})=0\right\}$.

Proof. The exact same proof will work as in the Characterization of $A(\alpha)$, provided that we can show that $M^{\prime}(\alpha)^{*}$ consists of all symbolic loops or quasi-loops $\underline{m}$ such that $u_{\alpha}(m)=0$. To do this, we need some kind of induction argument for showing that any $\underline{m}$ satisfying $u_{\alpha}(\underline{m})=0$ can be obtained by splicing simpler loops or quasi-loops. For the induction argument, we shall define a notion of the 'complexity' of a symbolic quasi-loop, and show that it behaves well under splicing and singular splicing.

Consider a symbolic quasi-loop $\underline{m}=\left(\underline{m}^{j}\right)_{j \in \mathbf{Z} / J}$. First define length $\left(\underline{m}^{j}\right)$ for $j \in \mathbb{Z} / J$. If the positive and negative tails of $\underline{m}^{j}$ are $\left(m_{i}^{j}\right)_{i \geq I}$ and $\left(m_{i}^{j}\right)_{i \leq I}$, then the length of $\underline{m}^{j}$ is $I-I^{\prime}$. Note that $\underline{m}_{-}^{j}$ and $\underline{m}_{+}^{j}$ are either identical or disjoint, so from the non-periodicity of $\underline{m}^{j}$ it follows that $I>I^{\prime}$, thus length $\left(\underline{m}^{j}\right)>0$.

Now define the complexity of $\underline{m}$ to be the ordered pair $\left(J, \sum_{j \in Z / J}\right.$ length $\left.\left(\underline{m}^{j}\right)\right)$. Complexities are given the dictionary ordering, and evidently form a well-ordered set. In order to make the induction argument work properly, we need to investigate how complexities behave with respect to splicing of loops and quasi-loops, and how complexities behave for simple loops and quasi-loops.

First we extend the notion of complexity to symbolic loops by defining the complexity of $\underline{m}=\left(m_{i}\right)_{i \in \mathbf{Z} / I}$ to be the ordered pair $(0, I)$.

Now we say now complexity behaves with respect to splicing:

LEMMA 4.4. Let $\underline{m}, \underline{m}^{\prime}$ be symbolic loops or quasi-loops, and let $C=\mathrm{Com}$ plexity (Splice $\left(\underline{m}, \underline{m}^{\prime}\right)$ ) or Complexity $\left(\operatorname{Splice}^{s}\left(\underline{m}, \underline{m}^{\prime}\right)\right.$ ) (assuming that the appropriate spliced loop is defined). Then $C \geq \sup \{$ Complexity $(m)$, Complexity $\left.\left(\underline{m}^{\prime}\right)\right\}$, with equality if and only if (up to a reversal of roles of $\underline{m}$ and $\underline{m}^{\prime}$ ) $\underline{m}$ is a symbolic quasi-loop whose splicing site is located in a tail of $\underline{m}^{0}$, and $\underline{m}^{\prime}$ is an iterate of the period loop of that tail

Proof. If $\underline{m}$ and $\underline{m}^{\prime}$ are both symbolic loops, this is obvious.

If $\underline{m}=\left(\underline{m}^{j}\right)_{j \in \mathbf{Z} / J}$ and $\underline{m}^{\prime}=\left(\underline{m}^{j}\right)_{j \in \mathbf{Z} / J}$ are both quasi-loops, then Splice $\left(\underline{m}, \underline{m}^{\prime}\right)$ and Splice $^{s}\left(\underline{m}, \underline{m}^{\prime}\right)$ are both made up of $J+J^{\prime}$ pieces, so the complexity is strictly larger. 
If $\underline{m}$ is a quasi-loop and $\underline{m}^{\prime}$ is a loop, then Splice ( $\left.\underline{m}, \underline{m}^{\prime}\right)$ is made up of the same number of pieces as $\underline{m}$, and we need only compare the length of piece 0 of $\underline{m}$ and piece 0 of Splice $\left(\underline{m}, \underline{m}^{\prime}\right)$. We consider several cases, depending on the location of the splicing site of $\underline{m}$.

Suppose the splicing site of $\underline{m}$ is not located in a periodic tail of $\underline{m}^{0}$. Then the length of piece 0 of Splice $\left(\underline{m}, \underline{m}^{\prime}\right)$ exceeds the length of piece 0 of $\underline{m}$ by an amount equal to Length $\left(\underline{m}^{\prime}\right)$.

Suppose the splicing site of $\underline{m}$ is located in a tail of $\underline{m}^{0}$, say, the positive tail $\left(m_{i}^{0}\right)_{i \geq 1}$, and suppose that $\underline{m}^{\prime}$ is not an iterate of $\underline{m}_{+}^{0}$; by definition of the splicing site, we must have $I \leq 0$. Then the length of piece 0 of Splice $\left(\underline{m}, \underline{m}^{\prime}\right)$ exceeds the length of piece 0 of $\underline{m}$ by at least $|I|+1$. This uses the fact that the period loop of a tail of $\underline{m}^{0}$ is simple, and that the negative tail $\left(m_{i}^{0}\right)_{i \leq I}$, does not overlap the positive tail, i.e. $I^{\prime}<I$.

The only remaining case is that the splicing site of $\underline{m}$ is located in a periodic tail of $\underline{m}^{0}$ and $\underline{m}^{\prime}$ is an iterate of the period loop of that tail; then Splice $\left(\underline{m}, \underline{m}^{\prime}\right)=\underline{m}$, and the complexities are equal.

Now we examine how to use splicing to reduce the complexity of a non-simple symbolic quasi-loop:

LEMMA 4.5. If $\underline{m}$ is a symbolic quasi-loop which is not simple, then up to a cyclic permutation of indices, we can write $\underline{m}=\operatorname{Splice}\left(\underline{m}^{\prime}, \underline{m}^{\prime \prime}\right)$ or Splice $\left(\underline{m}^{\prime}, \underline{m}^{\prime \prime}\right)$, where $\underline{m}^{\prime}, \underline{m}^{\prime \prime}$ are symbolic loops or quasi-loops with strictly smaller complexity than $\underline{m}$.

Proof. Suppose first that some symbol occurs in two different pieces of $\underline{m}$, say, $m_{i}^{j}=m_{i}^{j^{\prime}}$, where $j \neq j^{\prime}$, and suppose furthermore that the following property holds:

(*) if $j^{\prime}=j+1$, then either $m_{i}^{j}$ is not located in the positive tail of $\underline{m}^{j}$, or $m_{i}^{j^{\prime}}$ is not located in the negative tail of $\underline{m}^{j^{\prime}}$.

Then up to a re-indexing of $\underline{m}$, we can write $\underline{m}=$ Splice $\left(\underline{m}^{\prime}, \underline{m}^{\prime \prime}\right)$, where $\underline{m}^{\prime}, \underline{m}^{\prime \prime}$ have fewer pieces than $\underline{m}$; the non-periodicity of the pieces of $\underline{m}^{\prime}$ and $\underline{m}^{\prime \prime}$ is guaranteed by $(*)$.

Suppose next that some symbol occurs twice in the same piece of $\underline{m}$, and one of the occurrences is not in a tail; applying the previous lemma completes the proof.

Finally, suppose that some symbol occurs in the positive and negative tail of the same piece $\underline{m}^{0}$ of $\underline{m}$, and there are no other symbol repetitions in $\underline{m}^{0}$. Then $\underline{m}$ must consist of more than one piece, for otherwise $\underline{m}$ is simple. Thus, $\left(\underline{m}^{0}\right)$ forms a symbolic quasi-loop with one piece, and we can write $\underline{m}=\operatorname{Splice}^{s}\left(\left(\underline{m}^{0}\right), \underline{m}^{\prime}\right)$, for some symbolic quasi-loop $\underline{m}^{\prime}$ with one fewer piece than $m$.

To complete the proof of Characterization of $A^{\prime}(\alpha)$, consider a symbolic quasi-loop $\underline{m}$ satisfying $u_{\alpha}(\underline{m})=0$. Using the previous lemma, if $\underline{m}$ is not already simple then we can write $\underline{m}=$ Splice $\left(\underline{m}^{\prime}, \underline{m}^{\prime \prime}\right)$ or Splice $\left(\underline{m}^{\prime}, \underline{m}^{\prime \prime}\right)$ for two symbolic loops or quasi-loops with smaller complexity than $\underline{m}$. The splice equation shows that $u_{\alpha}\left(\underline{m}^{\prime}\right)+$ $u_{\alpha}\left(\underline{m}^{\prime \prime}\right)=u_{\alpha}(\underline{m})=0$, and since both terms on the left hand side are non-negative, it follows that $u_{\alpha}\left(\underline{m}^{\prime}\right)=u_{\alpha}\left(\underline{m}^{\prime \prime}\right)=0$. By induction, it follows that $\underline{m}^{\prime}, \underline{m}^{\prime \prime} \in M^{\prime}(\alpha)^{*}$, and so $\underline{m} \in M^{\prime}(\alpha)^{*}$. 
Now we prove that $L^{q}(\alpha)$ has only finitely many chain components. Consider the set $S_{A^{\prime}(\alpha)} \subset S_{A}$, which is invariant under the shift map $\rho$, and let $T_{A^{\prime}(\alpha)}$ be the image of $S_{A^{\prime}(\alpha)} \times \mathbb{R}$ under the quotient map $S_{A} \times \mathbb{R} \rightarrow T_{\rho}$. Proposition 4.3 Characterization of $A^{\prime}(\alpha)$, together with the definition of $L^{q}(\alpha)$, show that $T_{A^{\prime}(\alpha)}$ is the pre-image of $L^{q}(\alpha)$ under the semi-conjugacy $Q: T_{\rho} \rightarrow M$. The following lemma tells us what the orbits of Susp $(\rho)$ in $T_{A^{\prime}(\alpha)}-T_{A(\alpha)}$ look like:

LEMMA 4.6. Given $x \in T_{A^{\prime}(\alpha)}-T_{A(\alpha)}, L_{+}(x)$ and $L_{-}(x)$ are both contained in $T_{A(\alpha)}$. Proof. Let $\underline{m}=\left(m_{i}\right)_{i \in Z} \in S_{A^{\prime}(\alpha)}-S_{A(\alpha)}$ be the symbol sequence of the orbit $x \cdot \mathbb{R}$, i.e. $x \cdot \mathbb{R}$ is the image of $\underline{m} \times \mathbb{R}$ under the quotient map $S_{A} \times \mathbb{R} \rightarrow T_{\rho}$. In order to show that $L_{+}(x) \subset T_{A(\alpha)}$, it suffices to show that there exists $\underline{n} \in S_{A(\alpha)}$ and an integer $K$ such that for all $i \geq K, m_{i}=n_{i}$. The proof that $L_{-}(x) \subset T_{A(\alpha)}$ is similar.

Since $\mathcal{M}$ is finite, there exists some integer $K$ such that $m_{K}$ is repeated infinitely often in the half-infinite sequence $\left(m_{i}\right)_{i \geq K}$. Thus, there exist symbolic loops $\underline{n}^{j}=$ $\left(n_{i}^{j}\right)_{i \in \mathbb{Z} / L(j)}$ such that $L(j) \rightarrow \infty$ as $j \rightarrow \infty$, and for each $j, m_{K+i}=n_{i}^{j}$ for $i=$ $0,1, \ldots, L(j)$. It suffices to show that for each $j, \underline{n}^{j} \in S_{A(\alpha)}$, which by Proposition 3.6 Characterization of $A(\alpha)$ is the same as $u_{\alpha}\left(\underline{n}^{j}\right)=0$.

Since $\underline{m} \in S_{A^{\prime}(\alpha)}$, then by Proposition 4.3 Characterization of $A^{\prime}(\alpha)$, for each $j$ there exists a periodic quasi-orbit $\underline{m}^{j}$ of $A^{\prime}(\alpha)$ such that $u_{\alpha}\left(\underline{m}^{j}\right)=0$ and such that the finite symbol sequence $\left(m_{K}, m_{K+1}, \ldots, m_{K+L(j)}\right)$ occurs in one of the pieces of $\underline{m}^{j}$. This shows that $\underline{m}^{j}$ can be written as Splice $\left(\underline{n}^{j}, \underline{m}^{\prime}\right)$ for some periodic quasi-orbit $\underline{m}^{\prime}$. From the splice equation it now follows in the usual manner that $u_{\alpha}\left(\underline{n}^{j}\right)=0$.

Since $T_{A^{\prime}(\alpha)}=Q^{-1}\left(L^{q}(\alpha)\right)$ and $T_{A(\alpha)}=Q^{-1}(L(\alpha))$, it follows directly from the lemma that each orbit of $\phi$ contained in $L^{q}(\alpha)-L(\alpha)$ is asymptotic in forwards and backwards time to $L(\alpha)$. Therefore, each chain component of $L^{q}(\alpha)$ contains a chain component of $L(\alpha)$. We have already proven that $L(\alpha)$ has only finitely many chain components, so this finishes the proof of:

Proposition 4.7. $L^{q}(\alpha)$ has only finitely many chain components under the restricted flow $\phi \mid L^{q}(\alpha)$.

\section{Pseudo-Anosov shadowing theory}

In this section, we shall develop a shadowing theory for pseudo-Anosov maps, and use it to prove Theorem 3.8 Characterization of the Chain Kernel of $\alpha$, which says that $L^{q}(\alpha)=R(\alpha)$.

The shadowing theory was inspired by Handel's papers [H1] and [H2] and Shub's book [Sh], and was developed with much moral support from Joe Christy. The notion of 'jumping across a singularity' and the lemma Visitors Enter and Leave Through Corridors is essentially due to Handel. The primary difference is that Handel's 'global shadowing' theory takes place in the universal cover of $S$, whereas we shall work directly on the surface $S$, following along more traditional lines as in the proof of the Shadowing Lemma taken from chapter 8 of Shub's book [Sh]. 
We work with the pseudo-Anosov return map $f$ for the distinguished section $S$ of the flow $\phi$. Recall the assumption that $f$ fixes its separatrices. A sequence $\underline{z}=\left(z_{i}\right)_{i \in Z}$ such that $f\left(z_{i}\right)=z_{i+1}$ is called an orbit of $f$; a cyclic orbit is similarly defined replacing $\mathbb{Z}$ with $\mathbb{Z} / I$ for some $I \geq 1$. An $\varepsilon$-chain of $f$ is a sequence $z=\left(z_{i}\right)_{i \in Z}$ such that $d\left(f\left(z_{i}\right), z_{i+1}\right)<\varepsilon$ for each $i \in \mathbb{Z}$; an $\varepsilon$-cycle is similarly defined replacing $\mathbb{Z}$ with $\mathbb{Z} / I$ for some $I \geq 1$.

A doubly indexed sequence of the form $\underline{x}=\left(x_{i}^{j} \mid i \in \mathbb{Z}, j \in \mathscr{J}\right)$ is called a quasi-orbit of $f$ if $\mathscr{J}$ is an interval in $\mathbb{Z}$ (finite, semi-infinite, or infinite), $\underline{x}^{j}$ is an orbit of $f$ for each $j \in \mathscr{J}$, and if $j, j+1 \in \mathscr{I}$, then there exists $s^{j} \in \operatorname{Sing}(f)$ such that:

$$
\lim _{i \rightarrow+\infty} x_{i}^{j}=s^{j} \text { and } \lim _{i \rightarrow-\infty} x_{i}^{j+1}=s^{j} \text {. }
$$

We describe this by saying that $\underline{x}$ passes over $s^{j}$ at $j$. A cyclic quasi-orbit is similarly defined by replacing $\mathscr{J}$ with $\mathbb{Z} / J$ for some $J \geq 1$. If $\underline{x}$ is a cyclic orbit, cyclic $\varepsilon$-chain, or cyclic quasi-orbit of $f$, we define $\operatorname{Susp}(\underline{x})$ to be the cyclic orbit, cyclic $(\varepsilon, 1)$ chain, or cyclic quasi-orbit of the suspension flow $\phi$, defined as follows: when $x$ is a cyclic orbit or quasi-orbit of $f$, the definition is obvious; when $x=\left(x_{i}\right)_{i \in Z / I}$ is a cyclic $\varepsilon$-chain of $f$, set $t_{i}=1$ for $1 \leq i \leq I$, and define $\operatorname{Susp}(\underline{x})=\left(x_{0}, x_{1}, \ldots, x_{I} ; t_{1}, \ldots, t_{I}\right)$.

We want to prove a shadowing lemma for pseudo-Anosov maps. The proof of the standard shadowing lemma fails, because of the presence of singular points of $f$. We shall show that if one restricts the notion of $\varepsilon$-chains by disallowing 'jumps across singularities', then the classical notion of shadowing by true orbits can be recovered. Then we shall generalize this by showing that an arbitrary $\varepsilon$-chain can be shadowed in a non-classical sense, using quasi-orbits, where a jump of the $\varepsilon$-chain across a singularity corresponds to the quasi-orbit passing over the same singularity. To make all this rigorous, the first task is to be precise about what it means for an $\varepsilon$-chain of $f$ to 'jump across a singularity'.

Given an open set $U \subset S$, suppose that there exists an open set $V \subset \mathbb{C}$, a homeomorphism from $U$ to $V$, and an $n \geq 2$, such that $\mathscr{F}_{s} \mid U$ and $\mathscr{F}_{u} \mid U$ are the pull-backs of the horizontal and vertical foliations of the quadratic differential $z^{n-2} \cdot d z$, and $\partial V$ is the union of $n$ horizontal and $n$ vertical leaf segments of $z^{n-2} \cdot d z$, i.e. $V$ is a curvilinear $2 n$-gon with convex angles. When this happens, we say that $U$ is an $\mathscr{F}_{s}-\mathscr{F}_{u}$ chart on $S$. We define semi-metrics $D_{s}$ and $D_{u}$ on $U$, where $D_{s}(x, y)$ is the minimum $\mathscr{F}_{s}$ measure of a path between $x$ and $y$ in $U$, and $D_{u}(x, y)$ is the minimum $\mathscr{F}_{u}$ measure of such paths. Note that for a leaf segment $\mathscr{C} \subset U$ of $\mathscr{F}_{s}$ connecting points $x$ and $y, D_{u}(x, y)$ is the $\mathscr{F}_{u}$ measure of $\mathscr{C}$; similarly for leaf segments of $\mathscr{F}_{u}$. Now define a metric $D$ on $U$ by $D(x, y)=\sup \left\{D_{s}(x, y), D_{u}(x, y)\right\}$; we call this the box metric on $U$. Given a point $x \in U$ and $\varepsilon>0$, consider the ball $B_{\varepsilon}(x)$ of radius $\varepsilon$ around $x$ in the box metric. If $\varepsilon$ is sufficiently small, the closure in $U$ of $B_{\varepsilon}(x)$, denoted $\bar{B}_{\varepsilon}(x)$, is compact. If $x$ is a regular point and $\varepsilon$ is sufficiently small, then $\bar{B}_{\varepsilon}(x)$ is a closed $\mathscr{F}_{s}-\mathscr{F}_{u}$ rectangle. If $x \in \operatorname{Sing}(f, n)$ for some $n \geq 3$, then $\bar{B}_{\varepsilon}(x)$ is called an $\mathscr{F}_{s}-\mathscr{F}_{u}$ polygon, or more precisely, an $\mathscr{F}_{s}-\mathscr{F}_{u} 2 n$-gon. In either case, we refer to $\bar{B}_{\varepsilon}(x)$ as a box neighborhood of $x$ in $U$.

Since $S$ is compact, there exists a finite covering $\mathcal{U}$ of $S$ by $\mathscr{F}_{s}-\mathscr{F}_{u}$ charts, and a number $r>0$, with the following properties: 
(1) for each $x \in S$ there exists $U_{x} \in \mathscr{U}$ such that $x \in U_{x}$, and either $D(x, y)<r$ for some $y \in \operatorname{Sing}(f) \cap U_{x}$, or $\bar{B}_{r}(x)$ is a box neighborhood of $x$ in $U_{x}$;

(2) for each $x \in \operatorname{Sing}(f)$, if $U \neq U_{x} \in \mathcal{U}$, then $\bar{B}_{r}(x) \cap U=\varnothing$.

Under these conditions, it is easy to see that there exists a metric $D$ on $S$ such that $D$ restricted to any $U \in \mathscr{U}$ is the box metric on $U$. Moreover, $D$ can be chosen so that if $x \in \operatorname{Sing}(f)$, or if $x \in S-\bigcup\left\{\bar{B}_{r}\left(x^{\prime}\right) \mid x^{\prime} \in \operatorname{Sing}(f)\right\}$, then the closed ball of radius $r$ around $x$ is precisely the box neighborhood $\bar{B}_{r}(x)$. We shall refer to $D$ as the box metric on $S$, and $r$ as the injectivity radius of $S$.

We shall define several subsets of $S$ associated with a singularity $x \in \operatorname{Sing}(f)$, which will be used to define 'jumping across the singularity $x$ '. These subsets are all illustrated in diagram 4. For each $\varepsilon>0$, define $d(\varepsilon)=\varepsilon \cdot \lambda /(\lambda-1)$; choose $\varepsilon$ so small that $\varepsilon+\lambda \cdot d(\varepsilon) \leq r$. Given $x \in \operatorname{Sing}(f)$, let $W_{x}=\bar{B}_{\varepsilon+\lambda \cdot d(\varepsilon)}(x)$, and let $V_{x}=$ $\bar{B}_{d(\varepsilon)}(x)$. Also let $N_{x}^{s}=\left\{y \in W_{x} \mid D_{s}(x, y) \leq d(\varepsilon)\right\}$, and let $N_{x}^{u}=$ $\left\{y \in W_{x} \mid D_{u}(x, y) \leq d(\varepsilon)\right\}$. Notice that $V_{x}=N_{x}^{s} \cap N_{x}^{u}$. The closures of the components of $N_{x}^{s}-V_{x}$ are called the stable corridors of $x$, and the closures of the components if $N_{x}^{u}-V_{x}$ are called the unstable corridors of $x$. If $C^{s}$ is a stable corridor and $C^{u}$ is an unstable corridor, then $C^{s} \cap C^{u}$ is either a single point or empty; in the former case, we say that $C^{s}$ and $C^{u}$ are adjacent, otherwise they are non-adjacent. Let $L_{x}^{s}$ denote the union of the stable singular separatrices in $V_{x}$, and let $L_{x}^{u}$ denote the union of the unstable singular separatrices in $V_{x}$. The closures of the components of $V_{x}-L_{x}^{u}$ are called the stable sectors of $x$, and the closures of the components of $V_{x}-L_{x}^{s}$ are called the unstable sectors. Is $K^{s}$ is a stable sector and $K^{u}$ is an unstable sector, and if $K^{s} \cap K^{u}=\{x\}$, then $K^{s}$ and $K^{u}$ are non-adjacent; otherwise, $K^{s}$ and $K^{u}$ are adjacent, and their intersection is an $\mathscr{F}_{s}-\mathscr{F}_{u}$ rectangle called a quadrant of $x$. Note that the stable corridors of $x$ are in 1-1 correspondence with the stable sectors of $x$, where $C^{s}$ and $K^{s}$ correspond when their intersection consists of an
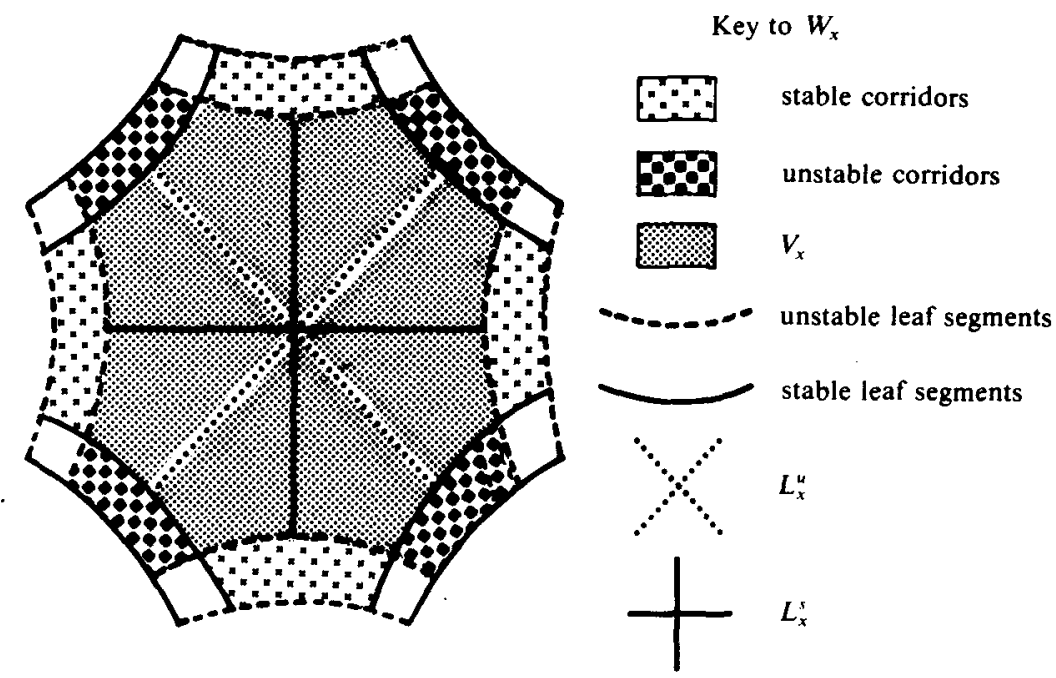

DiAgRAM 4. $W_{x}$ and the stable and unstable corridors of $x$. 
unstable leaf segment; otherwise, the intersection is empty. A similar statement holds for unstable corridors and sectors. Also, if $C^{s}, C^{u}$ are stable and unstable corridors with corresponding sectors $K^{s}$ and $K^{u}$, then $C^{s}$ and $C^{u}$ are adjacent if and only if $K^{s}$ and $K^{u}$ are adjacent, in which case the point $C^{s} \cap C^{u}$ is the corner of the quadrant $Q=K^{s} \cap K^{u}$ opposite the singularity $x$; we say that $Q$ is the quadrant corresponding to the adjacent corridors $C^{s}$ and $C^{u}$.

Notice that for all the sets defined in the previous paragraph, including $W_{x}, V_{x}$, stable and unstable corridors and sectors at $x$, and quadrants at $x$, the definitions depend on choosing some sufficiently small $\varepsilon>0$. Henceforth, the $\varepsilon$ used in the definitions will be clear from context.

Now fix some sufficiently small $\varepsilon>0$, and consider an $\varepsilon$-chain $z$ of $f$. Suppose that $z_{I} \in$ int $\left(V_{x}\right)$, for some $I \in \mathbb{Z}$ and $x \in \operatorname{Sing}(f)$. There exist unique $I_{-}, I_{+} \in \mathbb{Z} \cup$ $\{ \pm \infty\}$ so that $I^{-}<I<I^{+}, z_{i} \in$ int $\left(V_{x}\right)$ for $i \in\left(I^{-}, I^{+}\right)$, and $z_{I^{-}}, z_{I^{+}} \notin \operatorname{int}\left(V_{x}\right)$; we say that $\underline{z}$ visits the singularity $x$ in the interval $\left(I^{-}, I^{+}\right)$. By jiggling $z_{I^{-}}$and $z_{I^{+}}$slightly, we can assume that $z_{I^{-}}, z_{I^{+}} \notin V_{x}$, without altering the fact that $\underline{z}$ is an $\varepsilon$-chain.

Lemma 5.1. Visitors Enter and Leave Through Corridors. Suppose that the $\varepsilon$-chain $\underline{z}$ visits the singularity $x$ in the interval $\left(I^{-}, I^{+}\right)$. If $I^{-} \neq-\infty$, then $z_{I^{-}}$is contained in the interior of a stable corridor of $x$; and if $I^{+} \neq+\infty$, then $z_{l^{+}}$is contained in the interior of an unstable corridor of $x$.

Proof. We shall prove this statement for $I^{+} \neq+\infty$; the proof is similar for $I^{-} \neq-\infty$.

Recall that $f$ fixes all singularities and all singular separatrices. Notice that, since int $\left(V_{x}\right)=B_{d(\varepsilon)}(x)$, then $f\left(\right.$ int $\left.\left(V_{x}\right)\right) \subset B_{\lambda \cdot d(\varepsilon)}(x)$. In particular, since $z_{I^{+}-1} \in \operatorname{int}\left(V_{x}\right)$, and since $D\left(f\left(z_{I^{+}-1}\right), z_{I^{+}}\right)<\varepsilon$, we see that $z_{I^{+}} \in B_{\lambda \cdot d(\varepsilon)+\varepsilon}(x)=$ int $\left(W_{x}\right)$. Since $z_{I^{+}} \notin V_{x}$, it remains to show that $D_{u}\left(x, z_{I^{+}}\right)<d(\varepsilon)$.

Since $z_{I^{+}-1} \in$ int $\left(V_{x}\right)$, then $D_{u}\left(x, f\left(z_{I^{+}-1}\right)\right) \leq \lambda^{-1} \cdot d(\varepsilon)$. Since $D\left(f\left(z_{I^{+}-1}\right), z_{I^{+}}\right)<\varepsilon$, it follows that $D_{u}\left(f\left(z_{I^{+}-1}\right), z_{I^{+}}\right)<\varepsilon$, so $D_{u}\left(x, z_{l^{+}}\right)<\varepsilon+\lambda^{-1} \cdot d(\varepsilon)=\varepsilon+\varepsilon /(\lambda-1)=$ $\varepsilon \cdot \lambda /(\lambda-1)=d(\varepsilon)$.

Suppose now that the $\varepsilon$-chain $\underline{z}$ visits $x \in \operatorname{Sing}(f)$ in the interval $\left(I^{-}, I^{+}\right)$; suppose moreover that $I^{-} \neq-\infty, I^{+} \neq+\infty, C^{s}, C^{u}$ are the stable and unstable corridors through which $\underline{z}$ enters and leaves, and suppose that $C^{s}$ and $C^{u}$ are non-adjacent. Then we say that $\underline{z}$ jumps across the singularity $x$ in the interval $\left(I^{-}, I^{+}\right)$. Notice the following special case: when $I^{-}=-\infty$ or $I^{+}=+\infty$, then $\underline{z}$ does not jump across the singularity in the interval $\left(I^{-}, I^{+}\right)$.

Now we are ready to formulate our first pseudo-Anosov shadowing lemma. Given an $\varepsilon$-chain $\underline{z}$ of $f$, an orbit $\mathscr{\mathscr { S }}$ of $f$, and $\beta>0$, we say that $\underline{\mathscr{S}} \beta$-shadows $\underline{z}$ if $D\left(z_{i}, \mathscr{S}_{i}\right) \leq \beta$ for each $i \in \mathbb{Z}$; shadowing of an $\varepsilon$-cycle by a cyclic orbit of the same period is similarly defined.

Special Pseudo-Anosov Shadowing Lemma 5.2. For every $\beta>0$, there exists $\varepsilon>0$ such that for each $\varepsilon$-chain $\underline{z}$ of $f$, if $\underline{z}$ never jumps across a singularity of $f$, then there exists an orbit $\mathscr{S}$ of $f$ which $\beta$-shadows $\underline{z}$; if $\beta$ is sufficiently small, then $\mathscr{Q}$ is unique.

Proof. The proof follows very closely the proof of the standard Shadowing Lemma, which we take from p. 109 of Shub's book [Sh]. The set-up for the standard proof 
is as follows. If $R$ is an $\mathscr{F}_{u}-\mathscr{F}_{s}$ rectangle, then there is a product map $R \times R \rightarrow R$ defined as follows: given $x, y \in R$, the point $[x, y]$ is defined as the intersection of the stable leaf through $x$ in $R$, and the unstable leaf through $y$ in $R$. Also, given a regular point $x$ and a number $r>0$, let $\mathscr{C}_{r}^{s}(x)$ and $\mathscr{C}_{r}^{u}(x)$ denote the stable and unstable leaf segments of radius $r$ around $x$; in the pseudo-Anosov setting, this is defined as long as $x$ does not lie on a stable or unstable separatrix of a singularity $x^{\prime}$, within distance $r$ of $x^{\prime}$.

Consider first the problem of shadowing a finite $\varepsilon$-chain $\left(z_{0}, \ldots, z_{n}\right)$ by a finite orbit segment $\left(\mathscr{S}_{0}, \ldots, \mathscr{S}_{n}\right)$. Set $y_{0}=z_{0}$, and define $y_{k}$ recursively by the condition

$$
y_{k}=\left[z_{k}, f\left(y_{k-1}\right)\right] \text { for } 1 \leq k \leq n \text {. }
$$

In order for this to work, $z_{k}$ and $f\left(y_{k-1}\right)$ must be in some rectangle of small diameter so that the product map is uniquely defined; we shall check this condition momentarily. Now define $\mathscr{S}_{0}=f^{-n}\left(y_{n}\right)$, and $\mathscr{S}_{k}=f^{k}\left(\mathscr{S}_{0}\right)$ for $1 \leq k \leq n$; then $\left(\mathscr{S}_{0}, \ldots, \mathscr{S}_{n}\right)$ is our candidate for a finite segment of an orbit which shadows $\left(z_{0}, \ldots, z_{n}\right)$.

The proof given in Shub shows, even in the pseudo-Anosov case, that for every $\beta>0$ there exists $\delta>0$ and $\varepsilon>0$ such that for any finite $\varepsilon$-chain $\left(z_{0}, \ldots, z_{n}\right)$ of $f$, if $\mathscr{C}_{\delta}^{s}\left(f\left(z_{k-1}\right)\right)$ is defined and $\left[z_{k}, y\right]$ is defined for all $y \in \mathscr{C}_{\delta}^{s}\left(f\left(z_{k-1}\right)\right)$ and all $k=1, \ldots, n$, then the product in condition $(*)$ is defined, and the orbit segment $\left(\mathscr{S}_{0}, \ldots, \mathscr{S}_{n}\right) \beta$-shadows $\left(z_{0}, \ldots, z_{n}\right)$. From this it follows that if $\underline{z}$ is a bi-infinite $\varepsilon$-chain such that $\mathscr{C}_{\delta}^{s}\left(f\left(z_{k-1}\right)\right)$ is defined and $\left[z_{k}, y\right]$ is defined for all $y \in \mathscr{C}_{\delta}^{s}\left(f\left(z_{k-1}\right)\right)$ and all $k \in \mathbb{Z}$, then every finite segment of $\underline{z}$ is $\beta$-shadowed by a finite orbit segment; by taking limits we see that $z$ is $\beta$-shadowed by a true orbit.

Now let $\underline{z}$ be a bi-infinite $\varepsilon$-chain which never jumps across a singularity. The leaf segment $\mathscr{C}_{\delta}^{s}\left(f\left(z_{k-1}\right)\right)$ may not always be defined, or the product $\left[z_{k}, y\right]$ may not be defined for all $y \in \mathscr{C}_{\delta}^{s}\left(f\left(z_{k-1}\right)\right)$, but this problem can be overcome by making small corrections whenever $\underline{z}$ visits a singularity. Suppose that $\underline{z}$ visits a singularity $x$ in the interval $\left(I^{-}, I^{+}\right)$; we consider first the case where $I^{-} \neq-\infty$ and $I^{+} \neq+\infty$, so let $C^{s}, C^{u}$ be the stable and unstable corridors of $x$ such that $z_{I^{-}} \in C^{s}$ and $z_{J^{+}} \in C^{u}$. By hypothesis, $C^{s}$ and $C^{u}$ are adjacent; let $K^{s}$ and $K^{u}$ be the stable and unstable sectors corresponding to $C^{s}$ and $C^{u}$, and let $Q=K^{s} \cap K^{u}$ be the quadrant associated with the adjacent pair of corridors $C^{s}$ and $C^{u}$. If $i \in\left(I^{-}, I^{+}\right)$and $z_{i} \notin$ int $(Q)$, replace $z_{i}$ as follows: if $z_{i} \in K^{s}-Q$, replace $z_{i}$ by a point in int $(Q)$, very close to $\partial\left(K^{s}-Q\right)$, on the unstable leaf segment in $K^{s}$ containing $z_{i}$; if $z_{i} \in K^{u}-Q$, replace $z_{i}$ by a point in int $(Q)$, very close to $\partial\left(K^{u}-Q\right)$, on the stable leaf segment in $K^{u}$ containing $z_{i}$; and if $z_{i} \in V_{x}-\left(K^{s} \cup K^{u}\right)$, replace $z_{i}$ by a point in int $(Q)$ very close to $x$.

This process replaces the $\varepsilon$-chain $\underline{z}$ by a new $\varepsilon_{1}$-chain which stays in int $(Q)$ in the interval $\left(I^{-}, I^{+}\right)$, where $\varepsilon_{1}$ is small whenever $\varepsilon$ is small. Moreover, it is clear that the old chain is $d(\varepsilon)$-shadowed by the new chain. Thus, if the new chain is $(\beta-d(\varepsilon))$-shadowed by some orbit $\mathscr{\mathscr { }}$ of $f$, then the old chain is $\beta$-shadowed by $\underline{\varphi}$. Thus, we can assume that $z_{i} \in$ int $(Q)$ for $i \in\left(I^{-}, I^{+}\right)$. A similar replacement works when $\left(I^{-}, I^{+}\right)$is a half-infinite interval, say, $I^{+}=+\infty$ : in this case, $z_{l^{-}}$is in some stable corridor $C^{s}$ at $x$ with associated stable sector $K^{s}$, and it is easy to see that we can assume that $z_{i} \in$ int $\left(K^{s}\right)$ for all $i>I^{-}$(of course, when $I^{-}=-\infty$ and $I^{+}=+\infty$, then $\underline{z}$ is shadowed by the fixed point $x$ ). Clearly these replacements can be done 
simultaneously over all intervals $\left(I^{-}, I^{+}\right)$in which $z$ visits a singularity. With these additional assumptions on $\underline{z}$, it easily follows that for all sufficiently small $\delta>0$, $\mathscr{C}_{\delta}^{s}\left(f\left(z_{k-1}\right)\right)$ is defined, and $\left[z_{k}, y\right]$ is defined for all $y \in \mathscr{C}_{\delta}^{s}\left(f\left(z_{k-1}\right)\right)$ and all $k \in \mathbb{Z}$. This proves the existence part of the lemma.

Uniqueness is a direct consequence of the fact that a pseudo-Anosov map is expansive.

In order to get a general shadowing lemma, we must say what it means for an $\varepsilon$-chain $\underline{z}=\left(z_{i} \mid i \in \mathbb{Z}\right)$ of $f$ to be shadowed by a quasi-orbit $\underline{\mathscr{S}}=\left(\mathscr{S}_{i}^{j} \mid i \in \mathbb{Z}, j \in \mathscr{J}\right)$. Suppose that $\mathbb{Z}$ is partitioned into intervals $I(j)$, one for each $j \in \mathscr{J}$, in such a way that if $j<j^{\prime}, i \in I(j)$, and $i^{\prime} \in I\left(j^{\prime}\right)$, then $i<i^{\prime}$; if $j=\min (\mathscr{J})$, then $I(j)$ is negative semi-infinite, and if $j=\max (\mathscr{J})$, then $I(j)$ is positive semi-infinite; of course, if $|\mathscr{F}|=1$, then $I(j)=\mathbb{Z}$; otherwise, $I(j)$ is finite. Suppose also that we are given maps $k(j): I(j) \rightarrow \mathbb{Z}$ such that each $k(j)$ is a homomorphism, meaning that $k(j)(i+1)=$ $k(j)(i)+1$ whenever $i, i+1 \in I(j)$. Finally, suppose that for each $j \in \mathscr{J}$ and $i \in I(j)$, we have $D\left(z_{i}, \mathscr{S}_{k(j)(i)}^{j}\right)<\beta$. Then we say that $\mathscr{P} \beta$-shadows $\underline{z}$. Similarly, if $\underline{z}=\left(z_{i}\right)_{i \in Z / I}$ is an $\varepsilon$-cycle and $\mathscr{\mathscr { L }}$ is a quasi-orbit with $\mathscr{J}=\mathbb{Z} / J$, then shadowing can be defined by letting $(I(j) \mid j \in \mathbb{Z} / J)$ be a circularly ordered partition of $\mathbb{Z} / I$ into circular intervals; the rest of the definition of shadowing then goes through unchanged.

Also, uniqueness of shadowing must be formulated carefully. Given a quasi-orbit $\mathscr{\mathscr { S }}=\left(\mathscr{S}_{i}^{j} \mid i \in \mathbb{Z}, j \in \mathscr{J}\right)$ and $j \in \mathscr{I}$, notice that as $i \rightarrow+\infty, \mathscr{S}_{i}^{j}$ approaches the singularity $s^{j}$ along some stable separatrix $\mathscr{C}^{s}$; and as $i \rightarrow-\infty, \mathscr{P}_{i}^{j+1}$ approaches $s^{j}$ along an unstable separatrix $\mathscr{C}^{u} . \mathscr{C}^{s}$ and $\mathscr{C}^{u}$ are said to be adjacent if they bound some quadrant at $s^{j}$; otherwise, they are non-adjacent. If $\mathscr{C}^{s}$ and $\mathscr{C}^{u}$ are adjacent, then we say that the quasi-orbit $\mathscr{Q}$ has slack at $j$; and when $\mathscr{S}$ does not have slack at $j$ for any $j \in \mathscr{I}$, then $\mathscr{\mathscr { S }}$ is taut. Note that when $\mathscr{\mathscr { S }}$ has slack at $j$, then we can insert an $\varepsilon$-jump into $\mathscr{\mathscr { S }}$ near $s^{j}$ for some arbitrarily small $\varepsilon$. Doing this wherever $\mathscr{\mathscr { S }}$ has slack, we replace disjoint parts of $\mathscr{\mathscr { S }}$ of the form $\mathscr{\mathscr { g }}^{j} \cup \mathscr{\mathscr { Q }}^{j+1} \cup \cdots \cup \mathscr{\mathscr { Q }}^{j+k}$ by $\varepsilon$-chains, and then we can apply the Special Pseudo-Anosov Shadowing Lemma to these chains. This shows that an arbitrary quasi-orbit can be shadowed by a taut quasi-orbit, and this can be done so that the shadowing constant is arbitrarily small.

General Pseudo-Anosov Shadowing Lemma 5.3. For each $\beta>0$, there exists $\varepsilon>0$ such that for every $\varepsilon$-chain $\underline{z}$ of $f$, there exists a quasi-orbit $\mathscr{S}$ of $f$ which $\beta$-shadows z. Moreover, $\mathscr{S}$ can be taken to be taut, and if $\beta$ is sufficiently small, then a taut quasi-orbit which $\beta$-shadows $\underline{z}$ is unique.

Proof. Given $\beta>0$, choose $\varepsilon^{\prime}$ as in the Special Pseudo-Anosov Shadowing Lemma. Let $\underline{z}$ be an $\varepsilon$-chain with $\varepsilon<\varepsilon^{\prime}$; we will impose extra conditions on $\varepsilon$ momentarily. Choose an interval $\mathscr{J} \subset \mathbb{Z}$ and numbers $A^{j}<B^{j} \in \mathbb{Z} \cup\{ \pm \infty\}$ for $j \in \mathscr{I}$, so that $B^{j}<A^{j+1}$ for $j, j+1 \in \mathscr{I}, \mathscr{\mathscr { S }}$ visits a singularity $s^{j}$ in the interval $\left(B^{j}, A^{j+1}\right), \mathscr{\mathscr { S }}$ jumps across $s^{j}$ in this interval, and $\mathscr{\mathscr { S }}$ jumps across no other singularities. Since $\underline{z}$ is an $\varepsilon$-chain, then for $j, j+1 \in \mathscr{J}$ there must exist some $i(j) \in\left(B^{j}, A^{j+1}\right)$ such that $f\left(z_{i(j)}\right)$ and $z_{i(j)+1}$ are close to $s_{j}$; more specifically, by choosing $\varepsilon$ sufficiently small, clearly we can guarantee that $D\left(f\left(z_{i(j)}\right), s^{j}\right)<\varepsilon^{\prime}$ and $D\left(s^{j}, z_{i(j)+1}\right)<\varepsilon^{\prime}$. Now define $\varepsilon^{\prime}$-chains $\underline{z}^{j}$ for each $j \in \mathscr{J}$, by breaking $z$ between $i(j)$ and $i(j)+1$ whenever $j, j+1 \in \mathscr{I}$, adding 
infinitely many copies of $s^{j}$ after $i(j)$, and adding infinitely many copies of $s^{j}$ before $i(j)+1$. The Special Pseudo-Anosov Shadowing Lemma now applies, and $\underline{z}^{j}$ is $\beta$-shadowed by some orbit $\mathscr{\mathscr { S }}^{j}$ of $f$. Moreover, when $j, j+1 \in \mathscr{J}$, evidently $\mathscr{S}_{i}^{j}, \mathscr{S}_{-i}^{j+1} \rightarrow s^{j}$ as $i \rightarrow+\infty$. Thus, $\mathscr{\mathscr { S }}=\left(\mathscr{\mathscr { S }}^{j} \mid j \in \mathscr{J}\right)$ is a quasi-orbit which $\beta$-shadows $\underline{z}$, and evidently $\mathscr{\varphi}$ is taut.

To prove uniqueness, suppose $\mathscr{\varphi}^{\prime}=\left(\mathscr{\mathscr { S }}^{\prime j} \mid j \in \mathscr{J}^{\prime}\right)$ is another taut quasi-orbit which $\beta$-shadows $\underline{z}$. Thus, there is an order preserving partition $I^{\prime}(j), j \in \mathscr{J}^{\prime}$, such that $\left(z_{i}\right)_{i \in I^{\prime}(j)}$ is $\beta$-shadowed by a segment of $\underline{\mathscr{g}}^{\prime j}$ for $j \in \mathscr{J}^{\prime}$. Given $j, j+1 \in \mathscr{J}$ since $\underline{z}$ jumps across a singularity in the interval $\left(B_{j}, A_{j+1}\right)$, then there must exist some $j^{\prime} \in \mathscr{J}^{\prime}$ such that $\sup \left(I^{\prime}\left(j^{\prime}\right)\right), \inf \left(I^{\prime}\left(j^{\prime}+1\right)\right) \in\left(B_{j}, A_{j+1}\right)$. Also, given $j \in \mathscr{J}$, since $\underline{z}$ jumps across no singularity in any sub-interval of $\left(A_{j}, B_{j}\right)$, and since $\underline{\varphi}^{\prime}$ is taut, then there cannot exist any $j^{\prime} \in \mathscr{J}^{\prime}$ such that $\sup \left(I^{\prime}(j)\right) \in\left(A_{j}, B_{j}\right)$ or $\inf \left(I^{\prime}\left(j^{\prime}\right)\right) \in\left(A_{j}, B_{j}\right)$. From this it follows that there exists an order preserving bijection between $\mathscr{J}, \mathscr{J}^{\prime}$ such that when $j, j^{\prime}$ correspond under the bijection, then $\underline{\mathscr{S}}^{j}, \underline{\mathscr{S}}^{j^{\prime}}$ both $\beta$-shadow $\underline{z}^{j}$, so $\mathscr{S}^{j}=\mathscr{Q}^{j}$ by the uniqueness clause of the Special Pseudo-Anosov Shadowing Lemma. Therefore, $\underline{\mathscr{P}}=\mathscr{\mathscr { S }}^{\prime}$.

CoRollary 5.4. Given $\beta>0$ there exists $\varepsilon>0$ such that every $\varepsilon$-cycle $z$ of $f$ can be $\beta$-shadowed by a cyclic quasi-orbit $\mathscr{Y}$.

Proof. Let $\underline{z}$ be the periodic $\varepsilon$-cycle of $f$ covering $\underline{z}$. If $\varepsilon$ is as in the General Pseudo-Anosov Shadowing Lemma, then $\underline{\tilde{z}}$ is $\beta$-shadowed by some quasi-orbit $\tilde{\mathscr{S}}=\left(\mathscr{S}_{i}^{j} \mid i \in \mathbb{Z}, j \in \mathscr{J}\right)$. We say that a quasi-orbit is periodic if, under the dictionary ordering on $\mathscr{J} \times \mathbb{Z}$, there exists an order preserving bijection of $\mathscr{J} \times \mathbb{Z}$ which preserves points in the quasi-orbit. Clearly this is possible in a non-trivial way only if $\mathscr{g}=\mathbb{Z}$ or $|\mathscr{F}|=1$. By the uniqueness part of the General Pseudo-Anosov Shadowing Lemma, periodicity of $\tilde{z}$ implies periodicity of $\tilde{\mathscr{S}}$, so $\underline{\mathscr{Q}}$ covers a cyclic quasi-orbit $\underline{\mathscr{G}}$ of $f$ which $\beta$-shadows $\underline{z}$.

In order to relate the pseduo-Anosov shadowing theory to the study of the chain kernel $R(\alpha)$, we next show how $R(\alpha)$ is related to its intersection with the preferred section $S$ of the flow $\phi$.

LEMMA 5.5. Relating chains of $f$ and chains of $\phi$. Given $x \in S$, the following are equivalent:

(i) $x \in R(\alpha)$;

(ii) for every $\varepsilon>0$, there exists an $\varepsilon$-cycle $\underline{x}=\left(x_{i}\right)_{i \in \mathbf{Z} / I}$ of $f$, with $x=x_{0}$, such that $\langle\alpha, \operatorname{Susp}(\underline{x})\rangle=0$.

Proof. Suppose $x \in R(\alpha)$. Given $\varepsilon>0$ and $T>3$, let $X=\left(x_{i} ; t_{i}\right)_{i \in Z / I}$ be a periodic $\varepsilon, T$ cycle of $\phi$ such that $x=x_{0}$, and $\langle\alpha, X\rangle=0$. If $x_{i} \notin S$ for some $i$, let $x_{i}^{\prime}$ be the first point in $x_{i} \cdot[0, \infty) \cap S$, and suppose $x_{i}^{\prime}=x_{i} \cdot t_{i}^{\prime}$; thus, $0<t_{i}^{\prime}<1$. Now alter $X$ by replacing $x_{i}$ with $x_{i}^{\prime}$, and $t_{i}$ with $t_{i}-t_{i}^{\prime}+t_{i+1}^{\prime}$, for all $i \in \mathbb{Z} / I$. This replaces $X$ by an $\varepsilon^{\prime}, T^{\prime}$ chain $X^{\prime}$, where $\varepsilon^{\prime}$ differs from $\varepsilon$ by at most a bounded factor, and $T^{\prime}$ differs from $T$ by an additive constant of at most 2; moreover, clearly $\left\langle\alpha, X^{\prime}\right\rangle=\langle\alpha, X\rangle=0$.

Thus, we can assume that each $x_{i} \in S$. Under these conditions, if $\varepsilon$ is sufficiently small, then clearly each $t_{i}$ is very close to a positive integer, so we can assume that 
each $t_{i} \in \mathbb{Z}_{+}$. Now build an $\varepsilon$-chain of $f$ as follows: start with the circular sequence $\underline{x}=\left(x_{i}\right)_{i \in Z / I}$, and if $t_{i}>1$, replace $x_{i}$ with the sequence $x_{i}, f\left(x_{i}\right), \ldots f^{t_{i}-1}\left(x_{i}\right)$. This results in an $\varepsilon$-chain $\underline{x}^{\prime \prime}$ of $f$, where $x_{0}^{\prime \prime}=x_{0}=x$, and clearly $\left\langle\alpha, \operatorname{Susp}\left(\underline{x}^{\prime \prime}\right)\right\rangle=\langle\alpha, X\rangle=0$. This proves that property (i) implies property (ii).

Now assume that $x$ satisfies property (ii). Fix $\varepsilon>0$ and $T>0$. Let $N$ be the smallest integer $>2 T$. By uniform continuity of $f$, there exist positive numbers $\varepsilon(n)<\varepsilon$ defined for $0 \leq n \leq N$, and a number $\varepsilon^{\prime}>0$, such that if $0 \leq n<N, x, y, z \in S$, $D(x, y)<\varepsilon(n)$, and $D(f(y), z)<\varepsilon^{\prime}$, then $D(f(x), z)<\varepsilon(n+1)$. Choose an $\varepsilon^{\prime}$-cycle $\underline{x}=\left(x_{i}\right)_{i \in \mathbf{Z} / l}$ for $f$ with $x=x_{0}$ and $\langle\alpha$, Susp $(\underline{x})\rangle=0$. If $x$ is a periodic point and if $\alpha$ vanishes on the corresponding periodic orbit of $\phi$, there is nothing to prove; otherwise, by choosing $\varepsilon^{\prime}$ sufficiently small, we can assume that $I$ is large, say, $I>T$. This implies that there is a sub-sequence $0=i(0), i(1), \ldots, i(K-1), i(K)=I$ such that for each $1 \leq k \leq K$, setting $\Delta i(K)=i(K)-i(K-1)$, we have $T<\Delta i(K)<$ 2T. Also, it is clear that $D\left(f^{\Delta i(K)}\left(x_{i(K-1)}\right), x_{i(K)}\right)<\varepsilon(\Delta i(K))<\varepsilon$ for each $K$. This shows that $X=\left(x_{i(0)}, x_{i(1)}, x_{i(2)}, \ldots, x_{i(K)} ; \Delta i(1), \Delta i(2), \ldots, \Delta i(K)\right)$ is an $\varepsilon, T$ cycle of $\phi$ through $x$. Moreover, if $\varepsilon$ and $\varepsilon^{\prime}$ are sufficiently small it is clear that $\langle\alpha, X\rangle=$ $\langle\alpha, \operatorname{Susp}(\underline{x})\rangle=0$. Thus, $x \in R(\alpha)$.

The next proposition is the key to showing that if $x$ satisfies property (ii) of the previous proposition, then $x$ is approximated by periodic orbits or quasi-orbits in $L^{q}(\alpha)$ :

LeMmA 5.6. $\alpha$-Null Chains are Shadowed by $\alpha$-Null Orbits. Suppose that $\varepsilon$ and $\beta$ are small. Let $\underline{x}$ be an $\varepsilon$-cycle of $f$ which is $\beta$-shadowed by a cyclic quasi-orbit $\underline{Q}$ of $f$, and suppose that $\langle\alpha, \operatorname{Susp}(\underline{x})\rangle=0$. Then $\langle\alpha, \operatorname{Susp}(\underline{\mathscr{P}})\rangle=0$.

Before proving this lemma, we shall apply it to the

Proof of Theorem 3.8: Characterization of the Chain Kernel of $\alpha$. We want to show that $R(\alpha)$ and $L^{q}(\alpha)=L(\alpha) \cup \operatorname{cl}(\bigcup\{\mathscr{S} \mid \mathcal{S}$ is a periodic quasi-orbit of $\phi$ with $\langle\alpha, \mathscr{P}\rangle=0\})$ are the same sets. Since $R(\alpha)$ and $L^{q}(\alpha)$ are both closed invariant sets of the flow $\phi$, and since $S$ is a section of $\phi$, it suffices to show that $R(\alpha) \cap S=$ $L^{q}(\alpha) \cap S$. We have already shown that $L^{q}(\alpha) \subset R(\alpha)$, so $L^{q}(\alpha) \cap S \subset R(\alpha) \cap S$. It remains to show that $R(\alpha) \cap S \subset L^{q}(\alpha) \cap S$.

Given $x \in R(\alpha) \cap S$, the lemma Relating chains of $f$ and chains of $\phi$ shows that for any $\varepsilon$, there is an $\varepsilon$-cycle $\underline{x}$ of $f$ with $x=x_{0}$ and $\langle\alpha$, Susp $(\underline{x})\rangle=0$. By the corollary to the General Pseudo-Anosov Shadowing Lemma, given $\beta>0$ we can find such an $\underline{x}$ which is $\beta$-shadowed by some cyclic orbit or quasi-orbit $\mathscr{S}$ of $f$. If $\varepsilon$ and $\beta$ are small, then the lemma $\alpha$-Null Chains are Shadowed by $\alpha-N$ ull Orbits shows that $\langle\alpha$, Susp $(\mathscr{P})\rangle=0$, and it follows that $\operatorname{Susp}(\mathscr{\mathscr { S }}) \subset L^{q}(\alpha)$; thus, $\mathscr{\mathscr { C }} \subset L^{q}(\alpha) \cap S$. Also, if $\beta$ is small, then $\mathscr{Q}$ comes within $\beta$ of $x$. Since $L^{q}(\alpha) \cap S$ is closed and $\beta$ can be arbitrarily small, it follows that $x \in L^{q}(\alpha) \cap S$.

Proof. $\alpha$-Null Chains are Shadowed by $\alpha$-Null Orbits. Suppose $\underline{x}$ is an $\varepsilon$-cycle (small $\varepsilon)$ with $\langle\alpha, \operatorname{Susp}(\underline{x})\rangle=0$. Define a closed curve $C(\operatorname{Susp}(\underline{x}))$ by interpolating the jumps in Susp $(\underline{x})$ with short curves. If $\underline{x}$ never jumps across a singularity, and if $\underline{z}$ is a cyclic orbit of $f$ which $\beta$-shadows $\underline{x}$, then it is easy to see that $C(\operatorname{Susp}(\underline{x}))$ and 
$\operatorname{Susp}(\underline{z})$ are homotopic curves, so $\langle\alpha, \operatorname{Susp}(\underline{z})\rangle=\langle\alpha, C(\operatorname{Susp}(\underline{x}))\rangle=($ by definition $)$ $\langle\alpha, \operatorname{Susp}(\underline{x})\rangle=0$.

The difficulty arises when $\underline{x}$ jumps across some $s \in \operatorname{Sing}(f)$, in which case any cyclic quasi-orbit $\underline{z} \beta$-shadowing $\underline{x}$ will pass over $s$. Let $\mathscr{S}=\operatorname{Susp}(\underline{z})$, where $\mathscr{S}=$ $\left(\mathscr{S}^{j} \mid j \in \mathbb{Z} / J\right)$, and recall how $\langle\alpha, \mathscr{S}\rangle$ is defined. Build a closed curve $Z$ by choosing, for each $j \in \mathbb{Z} / J$, a very short curve $c^{j}$ which jumps from $\mathscr{S}^{j}$ to $\overline{\mathscr{S}}^{j}$ and then from $\overline{\mathscr{S}}^{j}$ to $\mathscr{S}^{j+1}$; if $\left\langle\alpha, \overline{\mathscr{S}}^{j}\right\rangle=0$ for each $j$, then $\langle\alpha, Z\rangle$ is independent of the choices made, and we define $\langle\alpha, \mathscr{S}\rangle=\langle\alpha, Z\rangle$. Otherwise, one defines $\langle\alpha, \mathscr{S}\rangle=+\infty$; and note that $\langle\alpha, Z\rangle \rightarrow+\infty$ as the length of the curves $c^{j}$ goes to zero.

Now notice that if $\varepsilon$ is sufficiently small, then the closed curve $Z$ can be chosen so that $Z$ is homotopic to Susp $(\underline{x})$. The point is that if $\underline{z}=\left(\underline{z}^{j} \mid j \in \mathscr{Z} / J\right)$ shadows $\underline{x}=\left(x_{i} \mid i \in \mathbb{Z} / I\right)$, with $(I(j) \mid j \in \mathbb{Z} / J)$ and $k(j): I(j) \rightarrow \mathbb{Z}$ as in the definition of shadowing, then $Z$ can be constructed as follows: for each $j$, let $m(j)=\min (I(j)), M(j)=$ $\max (I(j))$, and let $\mathscr{C}(j)$ be the oriented flow segment of $\phi$ connecting $z_{k(j)(m(j))}^{j}$ to $z_{k(j)(M(j))+1}^{j}$; then choose a short connecting path from the head of $\mathscr{C}(j)$ to the tail of $\mathscr{C}(j+1)$, which can be chosen short as long as $\varepsilon$ is small. The homotopy from Susp $(\underline{x})$ to $Z$ can be chosen to take $\mathscr{C}(j)$ to the path connecting $x_{m(j)}$ to $x_{M(j)+1}$, and the connection between $\mathscr{C}(j)$ and $\mathscr{C}(j+1)$ goes to the connecting path between $f\left(x_{M(j)}\right)$ to $x_{m(j+1)}$.

Thus, if we can show that $\left\langle\alpha, \overline{\mathscr{S}}^{j}\right\rangle=0$ for each $j \in \mathbb{Z} / J$, then it will follow that $\langle\alpha, \mathscr{S}\rangle=0$, because $\langle\alpha, \mathscr{S}\rangle=\langle\alpha, Z\rangle=\langle\alpha$, Susp $(\underline{x})\rangle=0$, where the last equation follows by homotopy of $Z$ and $\operatorname{Susp}(\underline{x})$.

It remains to show that $\left\langle\alpha, \overline{\mathscr{S}}^{j}\right\rangle=0$ for each $j \in \mathbb{Z} / J$. Let $Z$ be any closed curve constructed from $\mathscr{S}$ using a short path $c^{j}$ going from $\mathscr{S}^{j}$ to $\overline{\mathscr{S}}^{j}$ to $\mathscr{S}^{j+1}$. We shall show that for every $R>0$ there exists a number $\theta>0$ independent of $\mathscr{S}$, such that if length $\left(c^{j}\right)<\theta$ for each $j$, and if there exists $j$ with $\left\langle\alpha, \overline{\mathscr{S}}^{j}\right\rangle \neq 0$, then $\langle\alpha, Z\rangle>R$. But if $Z$ satisfies the conditions of the previous paragraph, then $\langle\alpha, Z\rangle=0$, so by taking $\varepsilon$ sufficiently small the jumps $c^{j}$ can be made smaller than $\theta$, and it follows that $\left\langle\alpha, \overline{\mathscr{Y}}^{j}\right\rangle=\mathbf{0}$ for each $j$.

Recall that $f$ fixes all singular separatrices. Let $\mathcal{M}^{*} \subset \mathcal{M}$ be the collection of all symbols $m$ such that $m \cap \operatorname{Sing}(f)=\{s\}$ for some $s$ with $\langle\alpha$, Susp $(s)\rangle \neq 0$; note that the unit length symbolic loop $\underline{m}=(m)$ satisfies the equation $O(\underline{m})=\operatorname{Susp}(s)$. Let $S^{*}$ denote the collection of all finite simple symbolic sequences $\gamma$ with Head $(\gamma)$, Tail $(\gamma) \in \mathcal{M}^{*}$, where Head $(\gamma) \cap$ Tail $(\gamma) \cap \operatorname{Sing}(f)=\{s(\gamma)\}$ for some singularity $s(\gamma)$ (recall that Head $(\gamma)$, Tail $(\gamma)$ are Markov boxes on the surface $S$ ). For each $\gamma \in S^{*}$, choose a closed curve $c(\gamma)$ in $M$ consisting of a flow line passing through each Markov box of $\gamma$ in sequence, closed off by some curve in $S$ which stays in Head $(\gamma) \cup$ Tail $(\gamma)$, passing through $s(\gamma)$. Let $L^{*}=\sup \left\{\langle\alpha, c(\gamma)\rangle \mid \gamma \in S^{*}\right\}$.

Take a cyclic quasi-orbit $\underline{z}=\left(\underline{z}^{j} \mid j \in \mathbb{Z} / J\right)$ of $f$, let $\mathscr{S}=\operatorname{Susp}(\underline{z})$, and let $\underline{m}$ be the symbolic quasi-loop such that $O(\underline{m})=\mathscr{S}$. Let $\mathscr{F}(\alpha)=\left\{j \in \mathbb{Z} / J \mid\left\langle\alpha, 0\left(\overline{\mathscr{S}}^{j}\right)\right\rangle \neq 0\right\}$. For each $j \in \mathscr{J}(\alpha)$, choose $m_{a(j)}^{j}$ in the positive tail of $\underline{m}^{j}$, and $m_{b(j)}^{j+1}$ in the negative tail of $\underline{m}^{j+1}$. If the positive tail of $\underline{m}^{j}$ is truncated after $m_{a(j)}^{j}$, and the negative tail of $\underline{m}_{b(j)}^{j+1}$ is truncated before $\boldsymbol{m}_{b(j)}^{j+1}$, and if this is done for each $j \in \mathscr{J}(\alpha)$, then the sum of $u_{\alpha}$ over the remainder of $\underline{m}$ is finite. Moreover, since $\left\langle\alpha, \overline{\mathscr{S}}^{j}\right\rangle$ is positive, then by 
choosing $a(j)$ sufficiently large, the sum of $u_{\alpha}$ over the remainder of $\underline{m}$ will be greater than $R$. Assuming that $a(j)$ is so chosen, choose $m_{c(j)}^{j}$ beyond $m_{a(j)}^{j}$ in the positive tail of $\underline{m}^{j}$ so that $u_{\alpha}\left(m_{a(j)}^{j}, m_{a(j)+1}^{j}, \ldots, m_{c(j)}^{j}\right)>L^{*}$; we say that the functions $a, b, c: \mathscr{I}(\alpha) \rightarrow \mathbb{Z}$ determine an $\left(R, L^{*}\right)$-truncation of $\underline{m}$.

We claim that there exists $\theta>0$, independent of $\underline{z}$, such that if $b, c: \mathscr{J}(\alpha) \rightarrow \mathbb{Z}$ are chosen so that $D\left(z_{b(j)}^{j}, z_{c(j)}^{j+1}\right)<\theta$ for each $j \in \mathscr{J}(\alpha)$, then there exists $a: \mathscr{J}(\alpha) \rightarrow \mathbb{Z}$ so that $a, b, c$ determine an $\left(R, L^{*}\right)$-truncation of $\underline{m}$. The reason is that in order for $D\left(z_{b(j)}^{j}, z_{c(j)}^{j+1}\right)$ to be very small, both $z_{b(j)}^{j}$ and $z_{c(j)}^{j+1}$ must be very close to some $s \in \operatorname{Sing}(f)$, where $\langle\alpha, \operatorname{Susp}(s)\rangle \neq 0$; but there is a positive lower bound on the distance from $s$ to any Markov box in $\mathcal{M}$ not containing $s$, so any orbit approaching very close to $s$ must spend a lot of time in the Markov boxes containing $s$; since $\langle\alpha, \operatorname{Susp}(s)\rangle \neq 0$, then during the time the orbit spends near $s$, the $u_{\alpha}$ sum of the Markov boxes visited by the orbit will build up very large. A precise formula for $\theta$ in terms of $R$ and $L^{*}$ would therefore involve the lengths of the sides of Markov boxes in $\mathcal{M}$, and the expansion factor $\lambda$, which determines how fast an orbit can move in a neighborhood of a singularity; we leave the curious reader to develop such a formula.

Now let $a, b, c$ be as in the previous paragraph; we must show that if $Z$ is the closed curve defined from $z$ by jumping from $m_{b(j)}^{j}$ to $m_{c(j)}^{j+1}$ for $j \in \mathscr{G}(\alpha)$, (with an arbitrary tiny jump for $j \notin \mathscr{J}(\alpha))$, then $\langle\alpha, Z\rangle\rangle R$. Let $\underline{n}$ be a cyclic quasi-loop obtained from $\underline{m}$ as follows: for each $j \in \mathscr{J}(\alpha)$, truncate $\underline{m}^{j}$ after $z_{b(j)}^{j}$, truncate $\underline{m}^{j+1}$ before $z_{c(j)}^{j}$, and splice in some $\gamma^{j} \in S^{*}$ going from $z_{b(j)}^{j}$ to $z_{c(j)}^{j+1}$. By an argument similar to the proof of the splice equation, we clearly have the following equation:

$$
\langle\alpha, Z\rangle+\sum_{j \in \mathscr{F}(\alpha)}\left\langle\alpha, c\left(\gamma^{j}\right)\right\rangle=\langle\alpha, \operatorname{Susp}(\underline{n})\rangle=u_{\alpha}(\underline{n}) .
$$

But since $a, b, c$ form an $\left(R, L^{*}\right)$-truncation of $\underline{m}$, it follows that $u_{\alpha}(\underline{n})>$ $R+|\mathscr{F}(\alpha)| \cdot L^{*}$. And by definition of $L^{*}$, we have $\left\langle\alpha, c\left(\gamma^{j}\right)\right\rangle\left\langle L^{*}\right.$ for each $j \in \mathscr{J}(\alpha)$. Thus, $\langle\alpha, Z\rangle>R$.

\section{Lifting the chain kernel}

Now we must show that each chain component of $R(\alpha)$ lifts to $\tilde{M}$. If $R(\alpha)$ were a locally path connected space, then we could apply the standard lifting lemma: since $\tilde{M} \rightarrow M$ is a $\mathbb{Z}$-cover defined by $\alpha \in H^{1}(M ; \mathbb{Z})$, we would only have to show that the image of $H_{1}(R(\alpha) ; \mathbb{Z})$ in $H_{1}(M ; \mathbb{Z})$ is contained in $\operatorname{Ker}(\alpha)$. As $R(\alpha)$ is not a locally path connected space, this does not work. To correct the situation, we shall find a neighborhood $N=N(R(\alpha))$ of $R(\alpha)$ with the property that image $\left(H_{1}(N ; \mathbb{Z}) \rightarrow H_{1}(M ; \mathbb{Z})\right) \subset \operatorname{Ker}(\alpha)$. Since $N$ is locally path connected, it follows that $N$ lifts to $\tilde{M}$, and $R(\alpha)$ goes along for the ride.

The neighborhood $N$ that we describe will be an isolating block for $R(\alpha)$. This is a concept due originally to Conley and Easton [CE], and is important in studying stability of closed invariant sets under perturbation of the flow. Recall the definition: given a flow on a manifold, a codimension 0 submanifold $N$ is called an isolating block if:

(i) $\partial N=S_{+} \cup S_{-}$, where $S_{+}, S_{-}$are codimension 0 submanifolds of $\partial N$; 
(ii) int $\left(S_{+}\right) \cap$ int $\left(S_{-}\right)=\varnothing$, and $\partial S_{+}=\partial S_{-}=\tau$, where $\tau$ is a codimension 1 submanifold of $\partial N$;

(iii) the flow enters $N$ in int $\left(S_{+}\right)$, leaves $N$ in int $\left(S_{-}\right)$, and is externally tangent to $N$ along $\tau$, i.e. for each $x \in \tau$, there exists $\varepsilon>0$ such that $x \cdot[-\varepsilon,+\varepsilon] \cap N=$ $\{x\}$.

Letting $I$ be the largest closed invariant set contained in int $(N)$, then $N$ is said to be an isolating block for $I$.

First we shall state and prove the relevant proposition in the case that $R(\alpha)$ contains no singular orbit of $\phi$. Once this is done, we shall indicate the changes necessary to deal with the other case.

Proposition 6.1. An Isolating Block for the Chain Kernel (non-singular case). Suppose that $R(\alpha)$ does not contain a singular orbit of $\phi$. then there exists an isolating block $N(\alpha)$ for $R(\alpha)$ with the following property: the digraph $\Gamma(\alpha)$ can be embedded as a deformation retract of $N(\alpha)$, in such a way that for each symbolic loop $\underline{m}$ of $A(\alpha)$, if $O(\underline{m})$ is the corresponding periodic orbit in $R(\alpha)$ and if $\gamma(\underline{m})$ is the corresponding directed loop in $\Gamma(\alpha)$, then $O(\underline{m})$ and $\gamma(\underline{m})$ are homotopic.

Before giving the proof, we use it to prove:

COROllary 6.2. The chain kernel lifts to $\tilde{M}$. Given a chain component $C$ of $R(\alpha)$, $x \in C$, and $\tilde{x} \in \tilde{M}$ lying over $x$, there exists a unique lifting of $C$ to $\tilde{M}$ containing $\tilde{x}$.

Proof. By the above proposition, it suffices to show that image $\left(H_{1}(N(\alpha) ; \mathbb{Z}) \rightarrow\right.$ $H_{1}(M ; \mathbb{Z})$ ) is contained in $\operatorname{Ker}(\alpha)$. Since $\Gamma(\alpha) \subset \operatorname{int}(N)$ is a deformation retract of $N$, it suffices to show that image $\left(H_{1}(\Gamma(\alpha) ; \mathbb{Z}) \rightarrow H_{1}(M ; \mathbb{Z})\right)$ is contained in $\operatorname{Ker}(\alpha)$. By Lemma 3.1 Directed Loops Span Homology in Strongly Connected Digraphs, we need only show that for each directed loop $\gamma \subset \Gamma(\alpha),[\gamma] \in \operatorname{Ker}(\alpha)$. Clearly there exists a symbolic loop $\underline{m}$ of $\boldsymbol{A}(\alpha)$ such that $\gamma=\gamma(\underline{m})$. By definition of $R(\alpha)$, it follows that $[O(\underline{m})] \in \operatorname{Ker}(\alpha)$. Using the Proposition 6.1, $\gamma$ and $O(\underline{m})$ are homotopic, so it follows that $[\gamma]=[O(m)] \in \operatorname{Ker}(\alpha)$, and we are done.

Proof of An Isolating Block for the Chain Kernel (non-singular version). Recall the standard notation: $S$ is the distinguished section of $\phi$, with pseudo-Anosov first return map $f$, stable and unstable foliations $\mathscr{F}_{s}, \mathscr{F}_{u}$, and expansion factor $\lambda>1$. Also, we assume that $\phi$ is parameterized so that the first return time to $S$ is everywhere equal to 1 .

Since $R(\alpha)$ contains no singular orbits, then $A(\alpha)=A^{\prime}(\alpha)$, and $R(\alpha)=L(\alpha)$ is the closure of the periodic orbits on which $\alpha$ vanishes, Let $\mathcal{M}(\alpha) \subset \mathscr{M}$ consist of all symbols occurring among the transitions in $A(\alpha)$. Consider a Markov rectangle $B \in \mathcal{M}$, so $B$ is an $\mathscr{F}_{s}, \mathscr{F}_{u}$ rectangle in $S$. Given $x, y \in B$, let $D_{s}(x, y)$ denote the measure of a path from $x$ to $y$ transverse to $\mathscr{F}_{u}$; for example, if $x, y$ lie on a segment of $\mathscr{F}_{s}, D_{s}(x, y)$ is the length of that segment measured with respect to $\mathscr{F}_{u}$. Let $D_{u}(x, y)$ be defined similarly. Let $D$ be the metric $D(x, y)=\sup \left\{D_{u}(x, y), D_{s}(x, y)\right\}$ on $B$. Let $\partial_{s} B$ denote the union of the two sides of $B$ consisting of leaf segments of $\mathscr{F}_{s}$; similarly for $\partial_{u} B$. Let $B^{+}=B \cdot[0,1]$ be the flow box of $\phi$ corresponding to $B$.

Given $B \in \mathcal{M}$, note that $B \in \mathcal{M}(\alpha)$ if and only if $R(\alpha) \cap B \neq \varnothing$. Since $R(\alpha) \cap S$ is 
a closed invariant set of $f$ containing no singularities, it follows that $R(\alpha) \cap S$ is disjoint from the stable and unstable separatrices of $f$. Since $\partial B$ is a union of segments of stable and unstable separatrices, if $B \in \mathcal{M}(\alpha)$ it follows that $R(\alpha) \cap$ $B \subset$ int $(B)$. In particular, there exists $\varepsilon>0$ such that $D(\partial B, R(\alpha) \cap B)>\varepsilon$. Since $\mathcal{M}(\alpha)$ is finite, $\varepsilon$ can be chosen independent of $B$.

The idea of the construction of $N$ is as follows. Given $B \in M(\alpha)$, imagine embedding a box $D=D(B)$ inside $B^{+}$, so that the top and bottom faces of $D$ are contained in the top and bottom faces of $B^{+}$, the vertical faces are parallel to the vertical faces of $B^{+}$, and $B^{+}-D$ consists of points whose horizontal distance to the vertical boundary of $B^{+}$is less that $\varepsilon$. Thus, int $(D)$ contains $B^{+} \cap R(\alpha)$. Consider the two vertical faces of $D$ which are parallel to the 'unstable' faces of $B^{+}$, i.e. the faces of $B^{+}$of the form $\sigma \cdot[0,1]$, where $\sigma \in \pi_{0}\left(\partial_{u}(B)\right)$; the union of these two faces will be denoted $\partial_{+} D . \partial_{-} D$ is similarly defined with respect to the 'stable' faces of $B^{+}$. Now perturb $D$ so as to skew the vertical faces, so that $\partial_{+} D$ slants outwards and $\partial_{-} D$ slants inwards. Thus, ignoring the top and bottom, the flow enters $D$ in int $\left(\partial_{+} D\right)$, leaves $D$ in int $\left(\partial_{-} D\right)$, and is externally tangent to $D$ along each vertical edge of $D$. $D$ is called a skewed box embedded in $B^{+}$. This is illustrated in diagram 5 , which includes a perspective view and a bird's eye view of $D$; in this diagram, unstable boundary components of $B$ are indicated with a dotted bold line, and stable boundary components of $B$ are indicated with a solid bold line. In the bird's eye view, the flow should be pictured as coming straight out of the page.
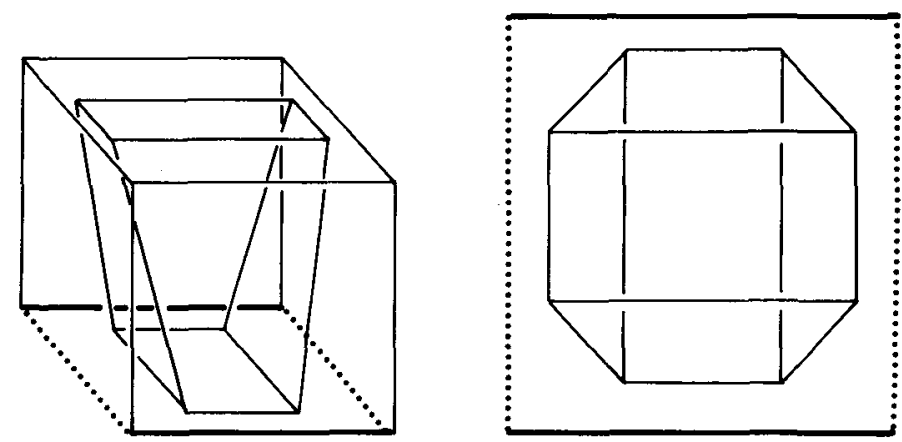

Diagram 5. A skewed box inside a flow box.

The isolating block $N$ will be constructed as a union of such skewed boxes $D(B)$, one inside $B^{+}$for each $B \in M(\alpha)$. However, the description given so far for the boxes $D(B)$ is not precise enough to avoid certain problems. For instance, if there exist $B, B^{\prime} \in \mathcal{M}(\alpha), \sigma \in \pi_{0}\left(\partial_{u} B\right)$ and $\sigma^{\prime} \in \pi_{0}\left(\partial_{u} B^{\prime}\right)$ such that $\left(B, B^{\prime}\right) \in A(\alpha)$ and $f(\sigma) \supset \sigma^{\prime}$, then we would want the corresponding top edge of $D(B)$ to contain the corresponding bottom edge of $D\left(B^{\prime}\right)$. In order to arrange for this to happen, we shall have to choose the boxes $D(B)$ more carefully.

Define $\pi_{0}\left(\partial_{s} M(\alpha)\right)$ to be the disjoint union of the sets $\pi_{0}\left(\partial_{s} B\right)$ for $B \in \mathcal{M}(\alpha)$; define $\pi_{0}\left(\partial_{u} \mathcal{M}(\alpha)\right)$ similarly. We shall refer to these two sets more succintly as $\pi_{0} \partial_{s}$ and $\pi_{0} \partial_{u}$, though the reader is cautioned that we are considering only edges of 
rectangles in $\mu(\alpha)$ as opposed to all of $\mu$. Define a directed graph with vertex set $\pi_{0} \partial_{s}$ and with a directed edge $\sigma \rightarrow \sigma^{\prime}$ whenever $\sigma \in \pi_{0}\left(\partial_{s} B\right) \subset \pi_{0} \partial_{s}, \sigma^{\prime} \in \pi_{0}\left(\partial_{s} B^{\prime}\right) \subset$ $\pi_{0} \partial_{s},\left(B^{\prime}, B\right) \in A(\alpha)$, and $\sigma^{\prime} \subset f^{-1}(\sigma)$; this directed graph is also denoted $\pi_{0} \partial_{s}$. Similarly, define a directed graph with vertex set $\pi_{0} \partial_{u}$ and directed edges $\sigma \rightarrow \sigma^{\prime}$ whenever $\sigma \in \pi_{0}\left(\partial_{u} B\right) \subset \pi_{0} \partial_{u}, \sigma^{\prime} \in \pi_{0}\left(\partial_{u} B^{\prime}\right) \subset \pi_{0} \partial_{u},\left(B, B^{\prime}\right) \in A(\alpha)$, and $\sigma^{\prime} \subset f(\sigma)$.

LEMMA 6.3. There exists a number $\nu>1$ and numbers $0<\varepsilon(\sigma)<\nu^{-1} \cdot \varepsilon$, defined for $\sigma \in \pi_{0} \partial_{s} \cup \pi_{0} \partial_{u}$, such that if $\sigma \rightarrow \sigma^{\prime}$ is a directed edge in $\pi_{0} \partial_{s}$ or $\pi_{0} \partial_{u}$, then $\nu \cdot \lambda \cdot \varepsilon\left(\sigma^{\prime}\right)=\varepsilon(\sigma)$.

Proof. This follows easily if it is true that each component of the directed graph $\pi_{0} \partial_{s}$ or $\pi_{0} \partial_{u}$ is a tree. Since each node of either digraph has at most one incoming directed edge, we need only show that there are no directed cycles. If there were a directed cycle of length $n$ in, say, $\pi_{0} \partial_{s}$, this would correspond to a symbolic loop $\left(B_{0}, B_{1}, \ldots, B_{n}=B_{0}\right)$ of $A(\alpha)$, and stable boundary components $\sigma_{i}$ of $B_{i}$, such that $f\left(\sigma_{i}\right) \subset \sigma_{i+1}$ for each $i \in \mathbb{Z} / n$, and $\sigma_{0}=\sigma_{n}$. Thus, $f^{n}\left(\sigma_{0}\right) \subset \sigma_{0}$, resulting in a periodic point $x \in \sigma_{0} ; x$ corresponds to the symbolic loop $\left(B_{i}\right)_{i \in Z / n}$ of $A(\alpha)$, implying that $x \in R(\alpha)$. However, each stable boundary component of a Markov box in $\mathcal{M}$ is a leaf segment of a stable separatrix based at a singularity. It follows that $x$ is a singularity of $f$. This contradicts the hypothesis that $R(\alpha)$ contains no singular orbits of $\phi$.

Now we can give precise definitions of the skewed boxes $D(B)$ and the isolating block $N$. Given $B \in M(\alpha)$, define $D(B) \subset B^{+}$to be the set of all points of the form $x \cdot t$, where $x \in$ int $(B)$ and $t \in[0,1]$ satisfying the following conditions:

(i) for each $\sigma \in \pi_{0}\left(\partial_{u} B\right), D(x, \sigma) \geq \varepsilon(\sigma) \cdot\left[(1-t)+t \cdot \nu^{-1}\right]$;

(ii) for each $\sigma \in \pi_{0}\left(\partial_{s} B\right), D(x, \sigma) \geq \varepsilon(\sigma) \cdot[\nu \cdot(1-t)+t]$.

Let Bottom $(D(B))$ be the rectangle consisting of all points $x=x \cdot 0$ of the above form, and let Top $(D(B))$ be the rectangle of all such points $x \cdot 1$. Let

$$
\partial_{+}^{h} D(B)=\operatorname{cl}\left[\text { Bottom }(D(B))-\bigcup\left\{\operatorname{Top}\left(B^{\prime}\right) \mid B^{\prime} \in \mathcal{M}(\alpha)\right\}\right],
$$

and let

$$
\partial_{-}^{h} D(B)=\operatorname{cl}\left[\operatorname{Top}(D(B))-\bigcup\left\{\operatorname{Bottom}\left(D\left(B^{\prime}\right)\right) \mid B^{\prime} \in M(\alpha)\right\}\right] ;
$$

these sets are the portions of the horizontal boundary of $D(B)$ not covered by horizontal boundaries of other boxes $D\left(B^{\prime}\right)$. Notice that because of the above lemma, $\partial_{+}^{h} D(B)$ is a union of sub-rectangles of Bottom $(D(B))$ each having stable boundary contained in the stable boundary of Bottom $(D(B))$, and $\partial_{-}^{h} D(B)$ consists of sub-rectangles of Top $(D(B))$ each having unstable boundary contained in the unstable boundary of Top $(D(B))$. Also, let $\partial_{+}^{v} D(B)$ be the union of the two outward slanting vertical faces, obtained by taking either of the two inequalities in condition (i) to be an equality; let $\partial_{-}^{v} D(B)$ be the inward slanting vertical faces, using equalities in condition (ii). Thus, the number $\nu$ is the slope of the faces $\partial_{+}^{v} D(B)$ and $\partial_{-}^{v} D(B)$, measured with respect to the parameterization of $B^{+}$as $B \times[0,1]$.

Now define $N=\bigcup\{D(B) \mid B \in M(\alpha)\}$, and define $S_{+} \subset \partial N$ to be the closure of the set $\bigcup\left\{\partial_{+}^{v} D(B) \cup \partial_{+}^{h} D(B) \mid B \in M(\alpha)\right\}$, and $S_{-} \subset \partial N$ to be the closure of the set $\bigcup\left\{\partial_{-}^{v} D(B) \cup \partial_{-}^{h} D(B) \mid B \in M(\alpha)\right\}$. It is evident that $S_{+}$and $S_{-}$are codimension 0 submanifolds of $\partial N, N=S_{+} \cup S_{-}$, the flow enters $N$ in int $\left(S_{+}\right)$, leaves $N$ in int $\left(S_{-}\right)$, 
and $\tau=S_{+} \cap S_{-}$is a codimension 1 submanifold. $\tau$ can be described as the union, over each $B \in M(\alpha)$, of the vertical edges of $D(B)$, plus the stable sides of $\partial_{+}^{h}(D(B))$, plus the unstable sides of $\partial_{-}^{h}(D(B))$. From this description, it is evident that the flow is externally tangent to $\tau$. Thus, $N$ is an isolating block.

This construction is illustrated in diagram 6, which shows how $N$ is constructed in a case where $A(\alpha)$ is a full shift on two symbols. In this example, $\mathcal{M}(\alpha)$ consists of two symbols $B_{0}$ and $B_{1}$, and $A(\alpha): \mathcal{M}(\alpha) \times \mathcal{M}(\alpha) \rightarrow\{0,1\}$ takes constant value 1 . In the diagram, the four horizontal sub-rectangles of Top $\left(B_{0}^{+}\right) \cup \operatorname{Top}\left(B_{1}^{+}\right)$labelled $R_{1}, R_{2}, R_{3}, R_{4}$ are identified with the four vertical sub-rectangles of Bottom $\left(B_{0}^{+}\right) \cup$ Bottom $\left(B_{1}^{+}\right)$labelled $R_{1}^{\prime}, R_{2}^{\prime}, R_{3}^{\prime}, R_{4}^{\prime}$. Since each such identification map is an orientation preserving affine homeomorphism, the map is completely specified by saying whether or not it preserves orientation of stable or unstable leaf segments. There are two possible choices for each of the four rectangle pairs $R_{i}, R_{i}^{\prime}$, making a total of $2^{4}=16$ possible choices in all. Note: each of the 16 choices defines a $\mathbb{Z} / 2$-valued cocycle on the digraph $\Gamma_{A(\alpha)}$, and two cocycles define the 'same' isolating block when they are in the same cohomology class; thus, since $H^{1}\left(\Gamma_{A(\alpha)} ; \mathbb{Z} / 2\right) \approx$ $(\mathbb{Z} / 2)^{3}$, the 16 possible choices only define 8 different isolating blocks.
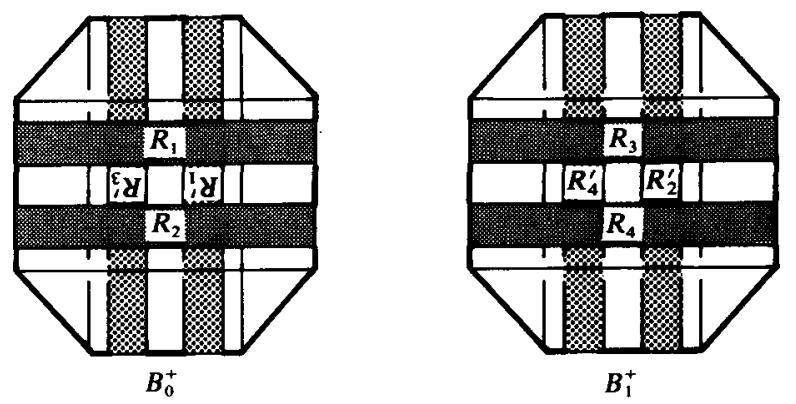

Diagram 6. An isolating block for the suspension of a full shift on two symbols.

One of the 16 examples is illustrated in diagram 6 , where the choices are indicated by the orientation of the labels $R_{i}^{\prime}, i=1,2,3,4$. The curve $\tau$ along which the flow is externally tangent is also indicated in the diagram by the bold-faced segments.

Now we prove the remaining contentions about $N$. It is evident that $R(\alpha) \subset$ int $(N)$. To see that $R(\alpha)$ is the largest invariant set in int $(N)$, consider an orbit $O(\underline{m})$ of $\phi$ not contained in $R(\alpha)$. Then for some symbol $m_{i}$ in $\underline{m}$, $m_{i}=B \notin \mathcal{M}(\alpha)$. Thus, $O(\underline{m})$ must intersect the flow box $B^{+}=B \cdot[0,1]$ for some $B \in M-M(\alpha)$; in fact, $O(m)$ must intersect $B \cdot(0,1)$. Since $B \cdot(0,1) \cap N=\varnothing$, it follows that $O(\underline{m}) \not \subset N$.

Now we describe how to embed $\Gamma(\alpha)$ in int $(N) . \Gamma(\alpha)$ has one node $n(B)$ for each $B \in \mathscr{M}(\alpha)$, and one directed edge $e\left(B, B^{\prime}\right)$ for each transition $\left(B, B^{\prime}\right) \in A(\alpha)$. Map $n(B)$ to an interior point of $D(B)$; map $e\left(B, B^{\prime}\right)$ to a path which connects $n(B) \in$ int $(D(B))$ to $n\left(B^{\prime}\right) \in$ int $\left(D\left(B^{\prime}\right)\right)$, passing through the rectangle Top $(D(B)) \cap$ Bottom $\left(D\left(B^{\prime}\right)\right)$. From this description, it is evident that $\Gamma(\alpha)$ is a deformation retract of $N$, and that for any symbolic loop $\underline{m}$ of $A(\alpha), O(\underline{m})$ and $\gamma(\underline{m})$ are homotopic. 
The proof of the above proposition yields a topological description of the isolating block $N$. The components of $N$ are in 1-1 correspondence with the components of $\Gamma(\alpha)$. If $C \in \pi_{0}(N)$ corresponds to $G \in \pi_{0}(\Gamma(\alpha))$, then $C$ is a handlebody, whose genus is given by the equation genus $(C)=1-\chi(G) . \tau$ is a family of simple closed curves on $\partial N$. For the example in the above diagram, it is an interesting exercise to draw what $\tau$ looks like on the boundary of a standard genus 3 handlebody in $\mathbb{R}^{3}$. Notice that the quadruple $\left(N, S_{+}, S_{-}, \tau\right)$ forms a sutured manifold in the terminology of Gabai [G]. This description in terms of sutured manifolds may be useful in obtaining relations between the flows we are considering here and the foliations studied by Gabai.

Now we shall indicate some of the changes necessary to prove that $R(\alpha)$ lifts when it contains singular orbits and quasi-orbits. It would be nice if we could replace the Markov partition $\mathcal{M}$ with a new Markov partition so that $R(\alpha)$ intersects a Markov rectangle $B \subset S$ only in int $(B)$. With the old notion of a Markov partition this is impossible, since int $(B)$ consists solely of regular points of $f$. To alleviate this problem, we alter our notion of a Markov rectangle, allowing a 'Markov 2 -gon' containing an $n$-pronged singularity in its interior.

Recall that the construction of a Markov partition for $f$, given in [FLP], started by choosing short stable separatrices for every singularity of $\mathscr{F}_{s}$. Instead, start with short stable separatrices only for those singularities of $\mathscr{F}_{s}$ in $S-R(\alpha)$. Continuing with the construction in [FLP], we obtain a partition of $S$ into ordinary Markov rectangles, plus, for each $n$-pronged singularity $s$ of $\mathscr{F}_{s}$ in $S \cap R(\alpha)$, a Markov $2 n$-gon $B_{s}$ with $s \in$ int $(B)$. This means that $\partial B_{s}$ consists of $n$ stable leaf segments and $n$ unstable leaf segments, alternating around the boundary. The collection of Markov rectangles and $2 n$-gons so obtained is called a pseudo-Markov partition, and denoted $\mathcal{M}^{p}$, with superscript $p$ for 'pseudo'. For each $B, B^{\prime} \in \mathcal{M}^{p}$, if $f($ int $(B)) \cap$ int $\left(B^{\prime}\right) \neq \varnothing$, the intersection $f(B) \cap B^{\prime}$ can be described as follows. If $B=B_{s}$ and $B^{\prime}=B_{s^{\prime}}$ for $n$-pronged singularities $s$, $s^{\prime}$ with $s^{\prime}=f(s)$, then $F(B) \cap B^{\prime}$ is a $2 n$-gon whose stable boundary is contained in the stable boundary of $B^{\prime}$, and whose unstable boundary is contained in the unstable boundary of $f(B)$. In all remaining cases, $f(B) \cap B^{\prime}$ is an ordinary rectangle, with the appropriate conditions on its stable and unstable boundaries. In diagram 7 , we illustrate a Markov $2 n$-gon $B_{s} \in \mathcal{M}^{p}$ for $n=4$; the diagram shows the two partitions of $B_{s}$ whose elements are of the form $B_{s} \cap f(B)$, and $B_{s} \cap f^{-1}(B)$ respectively, where $B$ ranges over the elements of $\mathscr{M}^{P}$.

Just as with the ordinary Markov partition $\mathcal{M}$, we define a transition matrix $A^{p}: \mathscr{M}^{p} \times \mathscr{M}^{p} \rightarrow\{0,1\}$ so that $A^{p}\left(B, B^{\prime}\right)=1$ if and only if $f($ int $(B)) \cap \operatorname{int}\left(B^{\prime}\right) \neq \varnothing$. Notice that a symbolic loop $\underline{m}$ of $A^{p}$ does not necessarily correspond to a periodic orbit of the suspension flow $\phi$. To see why, suppose $\underline{m}$ contains a sequence of the form $B_{0}, B_{s}, B_{1}$; thus, $f\left(B_{0}\right)$ interesects $B_{s}$ in some stable sector of $s$ and $f^{-1}\left(B_{1}\right)$ intersects $B_{s}$ in some unstable sector, and it may happen that these two sectors are not adjacent. However, it is evident in this case that $\underline{m}$ does correspond to a periodic quasi-orbit, denoted $O(\underline{m})$. Moreover, since the non-rectangular elements of $\mathcal{M}^{p}$ are in 1-1 correspondence with the elements of the set $\operatorname{Sing}(f) \cap R_{0}(\alpha)$, it follows 


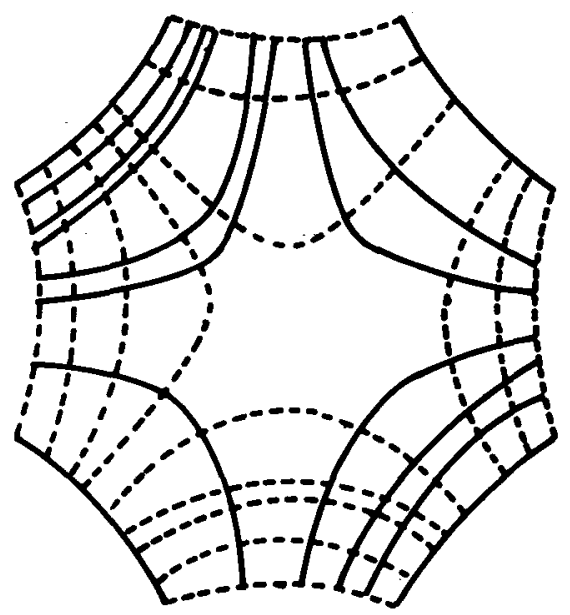

Diagram 7. A Markov octagon in a pseudo-Markov partition for a pseudo-Anosov homeomorphism.

that the periodic quasi-orbits of the form $O(\underline{m})$, where $\underline{m}$ ranges over the symbolic loops of $A^{p}$, consist precisely of those periodic quasi-orbits $O$ such that $\langle\alpha, O\rangle$ is finite.

Now we can carry through the analysis of $R(\alpha)$, using the pseudo-Markov partition $\mathcal{M}^{p}$ and transition matrix $A^{p}$. Define the sub-matrix $A^{p}(\alpha) \subset A^{p}$ to be the union of all symbolic loops $\underline{m}$ such that $\langle\alpha, O(\underline{m})\rangle=0$. Just as in $\S 5$, one now proves that $R(\alpha)$ consists of all orbits and quasi-orbits of the form $O(m)$ where $\underline{m}$ is a symbolic sequence of $A^{p}(\alpha)$. Let $\mathcal{M}^{p}(\alpha)$ denote those elements of $\mathcal{M}^{p}$ occurring among transitions in $A^{p}(\alpha)$. Just as in the non-singular case, $R(\alpha) \subset \bigcup\left\{B^{+} \mid B \in \mathcal{M}^{p}(\alpha)\right\}$. Utilizing this fact, we can construct an isolating block $N$ for $R(\alpha)$ as a union of skewed 'boxes' $D(B) \subset B^{+}$for each $B \in M^{p}(\alpha)$; when $B$ is a $2 n$-gon for $n>2$, then $D(B)$ is a $2 n$-gon crossed with an interval, with vertical faces slanting alternately inward and outward.

Now define the digraph $\Gamma^{p}$ to be the topological realization of $\boldsymbol{A}^{p}$, and show that there is a non-negative cohomology class $U^{p} \in H^{1}\left(\Gamma^{p} ; \mathbb{Z}\right)$ with the property that for each symbolic loop $\underline{m}$ of $A^{p}, U^{p}(\gamma(\underline{m}))=\langle\alpha, O(\underline{m})\rangle$. As before, one proves that the topological realization $\Gamma^{p}(\alpha)$ of $A^{p}(\alpha)$ is precisely the sub-digraph of $\Gamma^{p}$ consisting of the union of all directed loops $\gamma$ such that $U^{p}(\gamma)=0$. Now one proves, as before, that $\Gamma^{p}(\alpha)$ embeds as a deformation retract of $N$.

So in order to prove that $R(\alpha)$ can be lifted, it remains to show that $\langle\alpha, \gamma(\underline{m})\rangle=0$ for each symbolic loop $\underline{m}$ of $A^{p}(\alpha)$. Again, the proof is to find some kind of homotopy relation between $O(\underline{m})$ and $\gamma(\underline{m})$, but it is not immediately evident how to formulate such a relation, since $O(m)$ might not even be a closed curve, e.g. $O(\underline{m})$ might be a quasi-orbit. However, there is a canonical way to define a closed curve $O^{\prime}(\underline{m})$ well-defined up to homotopy, as follows. If $O(\underline{m})=\left(\mathscr{S}_{i}\right)_{i \in \mathbb{Z} / I}$, then for each $i$, there exists $s(i) \in R(\alpha) \cap \operatorname{sing}(f)$ such that $\overline{\mathscr{S}}_{i}=\operatorname{Susp}(s(i))$. Let $\mathscr{S}_{i}^{+}$be the non-compact component of $\mathscr{Y}_{i} \cap B_{s(i)}^{+}$representing the positive end of $\mathscr{Y}_{i}$, and let $\mathscr{S}_{i+1}^{-}$be the non-compact component of $\mathscr{S}_{i+1} \cap B_{s(i)}$ representing the negative end of $\mathscr{S}_{i+1}$. Then $\mathscr{S}_{i}^{+} \cup \overline{\mathscr{S}}_{i} \cup \mathscr{Y}_{i+1}^{-}$can be replaced by a path staying completely in $B_{s(i)}^{+}$ 
and having intersection number with $B_{s(i)}$ equal to the number of consecutive occurences of the symbol $B_{s(i)}$ in the appropriate interval of $\underline{m}$. Doing this replacement for each $i \in \mathbb{Z} / I$ results in a closed curve $O^{\prime}(\underline{m})$ well-defined up to homotopy, such that $\left\langle\alpha, O^{\prime}(\underline{m})\right\rangle=\langle\alpha, O(\underline{m})\rangle=0$. Moreover, the construction shows that $O^{\prime}(\underline{m})$ and $\gamma(\underline{m})$ are homotopic in $M$.

\section{Transient behavior of $\tilde{\phi}$}

Now define $\tilde{R}$ to be $p^{-1}(R(\alpha))$. It is evident that $\tilde{R} \subset \boldsymbol{R}(\tilde{\phi})$. In order to show the reverse inequality, it suffices to show that the remaining orbits of $\tilde{\phi}$ are transient. The following proposition does this and more:

Proposition 7.1. Transient Behaviour of $\tilde{\phi}$. Given $y \in \tilde{M}$, if $L_{ \pm}(y) \not \subset \tilde{R}$, then $\tilde{\phi}(y, t) \rightarrow$ $\pm \infty$ as $t \rightarrow \pm \infty$. Thus, $R(\tilde{\phi})=\tilde{R}=p^{-1}(R(\alpha))$.

From this proposition, together with the results of the previous sections, properties (A), (B), (C), and (D) of the $\mathbb{Z}$-Spectral Decomposition Theorem follow.

Proof of Proposition. We give two different arguments, both adapted from arguments of Fried.

The first argument uses a little shadowing theory, together with bits of the proof of Theorem D of [F2, pp. 357-358]. Suppose that $L_{+}(y) \not \subset \tilde{R}$ and $\tilde{\phi}(y, t)$ does not approach $+\infty$ as $t \rightarrow+\infty$. Then either $\tilde{\phi}(y, t) \rightarrow-\infty$ as $t \rightarrow+\infty$, or there is a point $z \in L_{+}(y)$ such that $z \notin \tilde{R}$.

First consider the case where $z \notin \tilde{R}$. Then for each $\varepsilon, T$, there exists an $\varepsilon, T$ cycle $X=(x, x ; t)$ such that $d(x, z)<\varepsilon$; this can be done by choosing $x=\tilde{\phi}(y, s)$ for some appropriately chosen $s$. Adapting our earlier shadowing arguments to the flow $\tilde{\phi}$, it follows that $X$ can be shadowed by a periodic orbit or quasi-orbit $\bar{X}$, and clearly the distance from $z$ to $\bar{X}$ is less than $K \cdot \varepsilon$, for some $K$ which is independent of $\varepsilon$. Thus, $z$ is in the closure of the collection of periodic orbits and quasi-orbits of $\tilde{\phi}$, from which it follows that $z \in \tilde{R}$, a contradiction.

Now consider the case where $\tilde{\phi}(y, t) \rightarrow-\infty$ as $t \rightarrow+\infty$. We can choose time $t_{i} \rightarrow+\infty$ such that $p\left(\tilde{\phi}\left(y, t_{i}\right)\right)$ has a limit point $z \in M$. Thus, for each $\varepsilon, T$ we can find an $\varepsilon$, $T$ chain $\left(z_{0}, z_{1} ; t\right)$ where $z_{i} \in p^{-1}(z)$, and where $z_{1}$ is significantly closer to $-\infty$ that $z_{0}$. Thus, $\left(p\left(z_{0}\right)=z, p\left(z_{1}\right)=z ; t\right)$ is an $\varepsilon, T$ chain downstairs having negative intersection number with $\alpha$. Again we employ a shadowing argument, deducing the existence of either a periodic orbit or a quasi-orbit of $\phi$ having negative intersection number with $\alpha$. This is a contradiction.

The first argument used shadowing, which depends on the pseudo-Anosov structure of the flow. For the second argument, we use the pseudo-Anosov structure of the flow in a different way, by employing the Markov partition; this proof also uses elements of the proof of Theorem $H$ of $[F 2$, p. 360]. Set $x=p(y)$, and choose a closed 1-form representing $\alpha$, which we also write as $\alpha$. Define $A_{x}: \mathbb{R} \rightarrow \mathbb{R}$ by $A_{x}(t)=\alpha(\partial / \partial t(\phi(x, t)))$. Then it suffices to show that:

$$
\int_{0}^{T} A_{x}(t) d t \rightarrow+\infty \text { as } T \rightarrow+\infty .
$$


We shall show this by estimating the integral by a sum of intersection numbers of $\alpha$ with closed curves which approximate long stretches of the flow line from $x$ to $\phi(x, T)$. From the fact that $L_{+}(y) \not \subset R(\tilde{\phi})$, it will follow that these closed curves have larger and larger intersection numbers with $\alpha$.

First of all, it suffices to assume that $x$ lies in our preferred section $S$ of $\phi$. Let $\underline{m}=\left(m_{i}\right)_{i \geq 0}$ be an allowable sequence of symbols visited successively by the flow line $x \cdot[0, \infty)$. Since $L_{+}(x) \not \subset R(\alpha)$, and since $R(\alpha)$ is covered by a particular subgraph $\Gamma$ of the symbolic dynamics graph of $\phi$, it follows that the symbolic path $\underline{m}$ is not eventually contained in $\Gamma$. That is, for each $I$, there exists integers $i, i^{\prime}$ with $I \leq i<i^{\prime}$ such that $m_{i}=m_{i^{\prime}}$, and the symbolic loop $\left(m_{i}, m_{i+1}, \ldots, m_{i^{\prime}}=m_{i}\right)$ has positive intersection number with $\alpha$.

Let $T_{k}=T(k)$ be an increasing sequence of numbers such that the flow segment $x \cdot\left[T_{k-1}, T_{k}\right]$ goes through a flow box from $m_{k-1}$ to $m_{k}$. Given $T \geq 0$, choose $k$ so that $T_{k} \leq T<T_{k+1}$. Clearly the integral

$$
\int_{T(k)}^{T} A_{x}(t) d t
$$

is bounded independent of $T$. Thus, to prove (*) we need only prove that:

$$
\int_{0}^{T(k)} A_{x}(t) d t \rightarrow+\infty \text { as } k \rightarrow+\infty
$$

Fix $K \geq 0$. Choose $0 \leq k(0) \leq K$ as large as possible so that $m_{0}=m_{k(0)}$; choose $k(1)$ with $k(0)<k(1) \leq K$ as large as possible so that $m_{k(0)+1}=m_{k(1)}$; etc. This generates a sub-sequence $k(0), k(1), \ldots, k(J)=K$, where $J$ is bounded by the number of symbols. Thus, the following quantity is bounded independent of $K$ :

$$
\left|\int_{0}^{T(K)} A_{x}(t) d t-\int_{0}^{T(k(0))} A_{x}(t) d t-\sum_{j=1}^{J} \int_{T(k(j-1)+1)}^{T(k(j))} A_{x}(t) d t\right| .
$$

Therefore, it suffices to prove that:

$$
\int_{0}^{T(k(0))} A_{x}(t) d t+\sum_{j=1}^{J} \int_{T(k(j-1)+1)}^{T(k(j))} A_{x}(t) d t \rightarrow+\infty \quad \text { as } K \rightarrow+\infty .
$$

Now choose a base point $p(m) \in m$ for each symbol $m$; for each $1 \leq j \leq J$, choose a path $\beta_{j}$ from $\phi\left(x, T_{k(j)}\right)$ to $p\left(m_{k(j)}\right)$; and for each pair of symbols $m, m^{\prime}$, choose a path $\beta_{m, m^{\prime}}$ in $M$ connecting $p(m)$ to $p\left(m^{\prime}\right)$; let $A$ be a constant bounding the integral of $\alpha$ over each of these paths. By concatenating these paths and their inverses appropriately with the flow segments $x \cdot[T(k(j-1)+1), T(k(j))]$, we see that summation in $(* * *)$ can be approximated to within $2 A(J+1)$ by

$$
\sum_{j=0}^{J}\left\langle\alpha, O\left(\underline{m}^{K, j}\right)\right\rangle,
$$

where $\underline{m}^{K, 0}$ is the symbolic loop $\left(m_{0}, \ldots, m_{k(0)}\right)$, and for $j=1, \ldots, J, \underline{m}^{K, j}$ is the symbolic loop $O\left(m_{k(j-1)+1}, m_{k(j-1)+2}, \ldots, m_{k(j)}\right)$. Thus, we need only show that the quantity $(* * * *)$ approaches $+\infty$ as $K \rightarrow+\infty$. Let $M^{K}$ be the indexed collection of simple symbolic loops obtained by desplicing all the loops in the 
list $\left(\underline{m}^{K, j} \mid j=0, \ldots, J\right)$, so we have

$$
\sum_{j=0}^{J}\left\langle\alpha, O\left(\underline{m}^{K, j}\right)\right\rangle=\sum_{m \in M^{K}}\langle\alpha, O(\underline{m})\rangle .
$$

Now the hypothesis that $L_{+}(x) \not \subset R(\alpha)$ implies that as $K \rightarrow+\infty$, the number of symbolic loops in the indexed collection $M^{K}$ whose orbits are not contained in $R(\alpha)$ goes to $+\infty$ as $K \rightarrow+\infty$; each such loop has an intersection number with $\alpha$ which is bounded below, and the proof is completed.

Using similar arguments for $T \rightarrow-\infty$, we are done.

\section{End behavior of $\tilde{\phi}$}

The last part of the $\mathbb{Z}$-Spectral Decomposition Theorem that needs verification is restated here for reference:

Property (E). Given $x \in \tilde{M}$, if $L_{+}(x) \neq\{+\infty\}$, then there exists a neighborhood $U$ of $+\infty$ such that for any chain component $C$ of $R(\tilde{\phi})$, if $C \subset U$ then $C \subset R_{+}(x)$.

We can think of ' $E$ ' as standing for 'End', and what this property says is that the flow $\tilde{\phi}$ behaves well near the ends of $\tilde{M}$.

We shall first recast property $\mathrm{E}$ as a purely combinatorial statement about the 'Liapounov graph' $\tilde{\Gamma}=\tilde{\Gamma}_{\alpha}$ of the flow $\tilde{\phi}$. $\tilde{\Gamma}$ is a digraph whose node set consists of the collection $\mathscr{C}$ of chain components of $R(\tilde{\phi})$. To define the directed edges of $\tilde{\Gamma}$, first we define a partial order on $\mathscr{C}$ by saying that for $C, C^{\prime} \in \mathscr{C}, C<C^{\prime}$ if there exists a sequence $C=C_{0}, C_{1}, \ldots, C_{n}=C^{\prime}$ in $\mathscr{C}$ and points $x_{1}, \ldots, x_{n}$ in $\tilde{M}-R(\tilde{\phi})$ such that $L_{-}\left(x_{i}\right) \subset C_{i-1}$ and $L_{+}\left(x_{i}\right) \subset C_{i}$, for $i=1, \ldots, n$. Notice that the partial order on $\mathscr{C}$ is non-reflexive, i.e. there does not exist any $C \in \mathscr{C}$ such that $C<C$; for otherwise, letting $C=C^{\prime}$ as above, since each $C_{i}$ is chain connected there would exist an $\varepsilon, T$ chain from a point in $L_{+}\left(x_{i}\right)$ to a point in $L_{-}\left(x_{i+1}\right)$ for each $i \in \mathbb{Z} / n$, and piecing these together with the orbits of the $x_{i}$ would give an $\varepsilon, T$ cycle through each $x_{i}$, contradicting the hypothesis that $x_{i} \in \tilde{M}-R$. Now define a directed edge $C \rightarrow C^{\prime}$ in $\tilde{\Gamma}$ whenever $C<C^{\prime}$ and there does not exist any $C^{\prime \prime} \in \mathscr{C}$ such that $C<C^{\prime \prime}<C^{\prime}$. Notice that $\tilde{\Gamma}$ is acyclic, i.e. there are no directed loops in $\tilde{\Gamma}$, for otherwise non-reflexivity of $\mathscr{C}$ would be contradicted.

Proposition 8.1. To prove property (E), it suffices to prove:

Property $\left(\mathrm{E}^{\prime}\right)$. For every $C \in \mathscr{C}=\operatorname{Nodes}(\tilde{\Gamma})$, there exists a neighborhood $V$ of $+\infty$ in $\tilde{\Gamma}$ such that for every $C^{\prime} \in V \cap \operatorname{Nodes}(\tilde{\Gamma})$, there is a directed path from $C$ to $C^{\prime}$ in $\tilde{\Gamma}$.

Proof. Assuming (E'), pick $x \in \tilde{M}$ such that $L_{+}(x) \neq\{+\infty\}$. We have already proven that $L_{+}(x) \subset C$ for some $C \in \operatorname{Nodes}(\tilde{\Gamma})$. Since the flow $\phi \mid C$ is chain recurrent, it follows that $R_{+}(x)=R_{+}(C)$. Clearly $R_{+}(C) \cap R(\tilde{\phi})=\bigcup\left\{C^{\prime} \in \operatorname{Nodes}(\tilde{\Gamma}) \mid\right.$ there exists a directed path in $\tilde{\Gamma}$ from $C$ to $C^{\prime}$. Also, for any sequence $C_{i} \in \operatorname{Nodes}(\tilde{\Gamma})$, $C_{i} \rightarrow+\infty$ in $\tilde{\Gamma}$ if and only $C_{i} \rightarrow+\infty$ in the Hausdorff topology of closed sets in $\tilde{\boldsymbol{M}} \cup\{ \pm \infty\}$. Thus, for each neighborhood $V$ of $+\infty$ in $\tilde{\Gamma}$, there exists a neighborhood $W$ of $+\infty$ in $\tilde{M}$ such that for $C^{\prime} \in \operatorname{Nodes}(\tilde{\Gamma})$, if $C^{\prime} \subset U$ then $C^{\prime} \in V$.

The next step is to convert property $\left(E^{\prime}\right)$ into a finitistic combinatorial statement. To accomplish this, notice that the action of $\mathbb{Z}$ on $\tilde{M}$ induces an action on $\tilde{\Gamma}$ by 
digraph isomorphisms, which is evidently free; we shall use $T$ to denote a generator of this group. Note that, as a topological space, $\tilde{\Gamma}$ can be compactified by adding ends $\{-\infty,+\infty\}$, in such a way that for each node $C$ of $\tilde{\Gamma}, T^{i}(C) \rightarrow \pm \infty$ as $i \rightarrow \pm \infty$. The quotient digraph $\Gamma=\Gamma_{\alpha}=\tilde{\Gamma} / \mathbb{Z}$ has only finitely many nodes; this is a consequence property (B) of the $\mathbb{Z}$-Spectral Decomposition Theorem, which has already been proven. The digraph $\Gamma$ comes equipped with a cohomology class $V=V_{\alpha} \in$ $H^{1}(\Gamma ; \mathbb{Z})$, which is naturally associated with the $\mathbb{Z}$-covering map $\tilde{\Gamma} \rightarrow \Gamma$.

Proposition 8.2. In order to prove property $\mathrm{E}^{\prime}$, it suffices to prove the following:

Property (E").

(i) $\Gamma$ is strongly connected;

(ii) let $L=\operatorname{gcd}\{V(\gamma) \mid \gamma$ is a closed, non-directed path in $\Gamma\}$. There exists $N \in \mathbb{Z}_{+}$ such that for each $n \geq N$ and for any $c \in \operatorname{Nodes}(\Gamma)$, there is a directed loop $\gamma$ through $c$ with $V(\gamma)=n \cdot L$.

Proof. Assuming property $\mathrm{E}^{\prime \prime}$, choose $C \in \operatorname{Nodes}(\tilde{\Gamma})$. Choose a cocycle $v \in V$, and let $\tilde{v}=q^{*}(v)$, where $q: \tilde{\Gamma} \rightarrow \Gamma$ is the projection map of the $\mathbb{Z}$-cover. We can write $\tilde{v}=d X$, where $X$ : Nodes $(\tilde{\Gamma}) \rightarrow \mathbb{Z}$ satisfies $X(C)=0$. Note that $X$ is a proper function in the sense that for a sequence $C_{i} \in \operatorname{Nodes}(\tilde{\Gamma}), C_{i} \rightarrow \pm \infty$ if and only if $X\left(C_{i}\right) \rightarrow \pm \infty$; this follows from connectivity of $\Gamma$, which follows from strong connectivity. So to prove property $E^{\prime}$, we need only prove that there exists some $M \in \mathbb{Z}_{+}$such that for each $C^{\prime} \in \operatorname{Nodes}(\tilde{\Gamma})$, if $X\left(C^{\prime}\right) \geq M$ then there is a directed path from $C$ to $C^{\prime}$ in $\tilde{\Gamma}$.

Let $c=q(C)$. For each $c^{\prime} \in$ Nodes $(\Gamma)$, choose a directed path $\gamma\left(c, c^{\prime}\right)$ from $c$ to $c^{\prime}$, using strong connectivity of $\Gamma$. Let $M=N \cdot L+\sup \left\{v\left(\gamma\left(c, c^{\prime}\right)\right) \mid c^{\prime} \in \operatorname{Nodes}(\Gamma)\right\}$; the sup exists because Nodes $(\Gamma)$ is finite. Suppose that $X\left(C^{\prime}\right) \geq M$ for $C^{\prime} \in$ Nodes $(\tilde{\Gamma})$; we must construct a directed path in $\tilde{\Gamma}$ from $C$ to $C^{\prime}$. Let $c^{\prime}=q\left(C^{\prime}\right)$. Lift $\gamma\left(c, c^{\prime}\right)$ to a path $\gamma\left(C_{1}, C^{\prime}\right)$ ending at $C^{\prime}$, and starting at some $C_{1} \in \operatorname{Nodes}(\tilde{\Gamma})$. Note that

$$
X\left(C_{1}\right)=X\left(C^{\prime}\right)-v^{*}\left(\gamma\left(C_{1}, C^{\prime}\right)\right)=X\left(C^{\prime}\right)-v\left(\gamma\left(c, c^{\prime}\right)\right) \geq N \cdot L .
$$

Notice also that $q\left(C_{1}\right)=q(C)=c$, so any (non-directed) path from $C$ to $C_{1}$ projects down to a closed loop; such paths exist by connectivity of $\tilde{\Gamma}$, and it follows that $X\left(C_{1}\right)=n \cdot L$ for some $n \geq N$. By hypothesis, there is a closed directed loop $\gamma$ through $c$ such that $v(\gamma)=V(\gamma)=n \cdot L$; let $\tilde{\gamma}$ be a lift of $\gamma$ starting at $C$. Clearly $\operatorname{Head}(\tilde{\gamma})=\operatorname{Tail}\left(\gamma\left(C_{1}, C^{\prime}\right)\right)=C_{1}$, so $\tilde{\gamma} * \gamma\left(C_{1}, C^{\prime}\right)$ is the desired directed path from $C$ to $C^{\prime}$.

We next show that property $E^{\prime \prime}$ is a formal consequence of strong connectivity of $\Gamma$ and positivity of $V$, which will be proven later.

Proposition 8.3. Given a finite strongly connected digraph $\Gamma$ and a positive cohomology class $V \in H^{1}(\Gamma ; \mathbb{Z}), \Gamma$ and $V$ satisfy property $\mathrm{E}^{\prime \prime}$.

Proof. As we have seen in the Lemma 3.1 Directed Loops Span Homology in a Strongly Connected Digraph, if $H \subset H_{1}(\Gamma ; \mathbb{Z})$ denotes the semi-group consisting of all positive linear combinations of homology classes of closed directed loops, then $\boldsymbol{H}$ generates all of $H_{1}(\Gamma ; \mathbb{Z})$. Thus, the set $V(H)$, the image of $H$ under the homomorphism 
$V: H_{1}(\Gamma ; \mathbb{Z}) \rightarrow \mathbb{Z}$, which is a semi-group of positive integers, generates all of image $(V)=L \cdot \mathbb{Z}$. By dividing through by $L$, we can assume $L=1$, and it remains to show that $V(H)$ contains all sufficiently large integers. This can be accomplished by techniques similar to but simpler than those used in the proof of Minkowski's theorem (see [HW], p. 31):

LEMMA 8.4. Let $S \subset \mathbb{Z}_{+}$be a semi-group which generates all of $\mathbb{Z}$. Then $S$ contains all sufficiently large integers.

Proof. By hypothesis, we can write

$$
1=\sum_{j=1}^{J} n_{j} \cdot s_{j}
$$

for some $n_{j} \in \mathbb{Z}$ and $s_{j} \in S$. Consider the linear map $F: \mathbb{R}^{J} \rightarrow \mathbb{R}$ given by

$$
F(x)=\sum_{j=1}^{J} n_{j} \cdot x_{j}
$$

Note that the restriction $F: \mathbb{Z}^{J} \rightarrow \mathbb{Z}$ is onto, since 1 is in the image. We shall show that for all sufficiently large $n, F^{-1}(n) \cap \mathbb{Z}_{+}^{J} \neq \varnothing$, from which the lemma follows.

Let $\Lambda=\operatorname{Ker}(F) \cap \mathbb{Z}^{J}$. Since $F$ is defined over $\mathbb{Z}, \Lambda$ is a lattice in $\operatorname{Ker}(F) \approx \mathbb{R}^{J-1}$, so the quotient torus $\operatorname{Ker}(F) / \Lambda$ is compact, and in particular has finite diameter. Therefore there exists a constant $A>0$ such that any ball in $\operatorname{Ker}(F)$ of radius $>A$ contains a point of $\Lambda$. Also, for any integer $n$, translating $\operatorname{Ker}(F)$ by some particular element of $F^{-1}(n) \cap \mathbb{Z}^{J}$ is an isometry from $\operatorname{Ker}(F)$ to $F^{-1}(n)$ taking $\Lambda$ to the lattice $F^{-1}(n) \cap \mathbb{Z}^{J}$. It follows that any ball in $F^{-1}(n)$ of radius $>A$ contains a point of $\mathbb{Z}^{J}$.

Consider the simplex $\sigma=F^{-1}(1) \cap \mathbb{R}_{+}^{K}$. Choose a ball $B \subset$ int $(\sigma)$, and let $r=$ radius $(B)$. Note that for every $n>A / r$, the simplex $F^{-1}(n) \cap \mathbb{R}_{+}^{J}$ contains the ball $n \cdot B$ of radius $n \cdot r>A$, which therefore contains a point $x \in \mathbb{Z}^{J}$. Thus, $x \in F^{-1}(n) \cap$ $\mathbb{R}_{+}^{J} \cap \mathbb{Z}^{J}=F^{-1}(n) \cap \mathbb{Z}_{+}^{J}$.

So to finish the proof of property (E) and of the $\mathbb{Z}$-Spectral Decomposition Theorem, it remains to prove that the digraph $\Gamma$ is strongly connected and the cohomology class $V \in H_{1}(\Gamma ; \mathbb{Z})$ is positive. For the remainder of the proof, we shall revert to the more specific notation $\Gamma_{\alpha}$ for $\Gamma$ and $V_{\alpha}$ for $V$.

As in the construction of an isolating neighborhood for the chain kernel $R(\alpha)$, the proof depends on whether or not $R(\alpha)$ contains a singular orbit of $\phi$. When no such singular orbit exists, we shall show how to construct $\Gamma_{\alpha}$ and $V_{\alpha}$ from the symbolic dynamics digraph $\Gamma_{\mathrm{A}}$ and the non-negative cohomology class $U_{\alpha} \in$ $H^{1}\left(\Gamma_{a}: \mathbb{Z}\right)$, defined in $\S 3$; from the construction, the desired properties of $\Gamma_{\alpha}$ and $V_{\alpha}$ will follow. When $R(\alpha)$ does contain a singular orbit of $\phi$, we shall outline a similar procedure which allows one to construct $\Gamma_{\alpha}$ and $V_{\alpha}$ from the directed graph $\Gamma^{p}$ and cohomology class $U^{p} \in H^{1}\left(\Gamma^{p} ; \mathbb{Z}\right)$ defined in $\S 5$.

Here is the construction for $\Gamma_{\alpha}$, assuming that $R(\alpha)$ contains no singular orbits of $\phi$, so $R(\alpha)=L(\alpha)$. As was proven in $\S 5$, the chain components of $R(\alpha)$ are in 1-1 correspondence with the topological components of the sub-digraph $\Gamma_{A(\alpha)} \subset \Gamma_{A}$. Let $u$ be a non-negative cocycle representing $U_{\alpha} \in H^{1}\left(\Gamma_{a} ; \mathbb{Z}\right)$, which exists because $U_{\alpha}$ is non-negative. Recall that $u$ takes value zero on each edge in $\Gamma_{A(\alpha)}$. 
Consider a directed path $\gamma$ in $\Gamma_{A}$ whose Head and Tail are in $\Gamma_{A(\alpha)} \cdot \gamma$ is said to be $\alpha$-indecomposable if $\gamma$ contains no edge of $\Gamma_{A(\alpha)}$, and $\gamma$ passes through no node of $\Gamma_{A(\alpha)}$ other than at Head $(\gamma)$ and Tail $(\gamma) . \gamma$ is $\alpha$-minimal if, given any path $\gamma^{\prime}$ such that $\partial \gamma=\partial \gamma^{\prime}$ and $u(\gamma)=u\left(\gamma^{\prime}\right), \gamma^{\prime}$ is $\alpha$-indecomposable. Thus, an $\alpha$-minimal path is $\alpha$-indecomposable, but the converse is not necessarily true. Define an equivalence relation on $\alpha$-minimal paths by saying that $\gamma \approx \gamma^{\prime}$ if Tail $(\gamma)$, Tail $\left(\gamma^{\prime}\right)$ are in the same component of $\Gamma_{A(\alpha)}$, Head $(\gamma)$ and Head $\left(\gamma^{\prime}\right)$ are in the same component of $\Gamma_{A(\alpha)}$, and $u(\gamma)=u\left(\gamma^{\prime}\right)$. Define a directed graph $\Gamma_{\alpha}^{\prime}$ with one node [C] for each component $C$ of $\Gamma_{A(a)}$, and one directed edge $[\gamma]$ for each equivalence class of $\alpha$-minimal paths $\gamma$; thus, Head $[\gamma]=[C]$ if and only if Head $(\gamma)$ is a node of $C$, and similarly for Tails.

Note, incidentally, that the definition of $\alpha$-minimal and the equivalence relation on $\alpha$-minimal paths depends only on the positive cohomology class $U_{\alpha}$, not on the non-negative cocycle $u$ representing $U_{\alpha}$. Also, note that the graph $\Gamma_{\alpha}^{\prime}$ comes naturally equipped with a non-negative cohomology class $V_{\alpha}^{\prime} \in H^{1}\left(\Gamma^{\prime}(\alpha) ; \mathbb{Z}\right)$, defined by the equation $V_{\alpha}^{\prime}\left(\left[\gamma_{1}\right] * \cdots *\left[\gamma_{K}\right]\right)=\sum_{k \in \mathbb{Z} / K} u\left(\gamma_{k}\right)$; it is easily checked that this is welldefined, and depends only on the cohomology class $U_{\alpha} \in H^{1}\left(\Gamma_{A} ; \mathbb{Z}\right)$. Moreover, $V_{\alpha}^{\prime}$ is positive. For suppose there is a directed loop $\left[\gamma_{1}\right] * \cdots *\left[\gamma_{K}\right]$ with $V_{\alpha}^{\prime}\left(\left[\gamma_{1}\right] * \cdots *\left[\gamma_{K}\right]\right)=0$. There are directed paths $\left(\beta_{k}\right)_{1 \leq k \leq K}$ in $\Gamma_{A(\alpha)}$ such that Head $\left(\gamma_{k}\right)=$ Tail $\left(\beta_{k}\right)$ and Head $\left(\beta_{k}\right)=$ Tail $\left(\gamma_{k+1}\right)$. Thus, $\gamma_{1} * \beta_{1} * \cdots * \gamma_{K} * \beta_{K}$ is a directed loop in $\Gamma_{A}$ whose value under $U_{\alpha}$ equals zero; as proven earlier, this loop is contained in $\Gamma_{A(\alpha)}$, which contradicts $\alpha$-minimality of the $\gamma_{k}$.

Strong connectivity of $\Gamma_{\alpha}^{\prime}$ follows from strong connectivity of $\Gamma_{A}$ as follows. Given two directed edges $\left[\gamma_{a}\right],\left[\gamma_{b}\right]$ of $\Gamma_{\alpha}^{\prime}$, choose a directed path in $\Gamma_{A}$ of the form $\gamma_{a} * \beta * \gamma_{b}$. Note that $\beta$ can be written as a concatenation of directed paths of the form $\gamma_{2} * \cdots * \gamma_{K-1}$, where each $\gamma_{k}$ either is contained in $\Gamma_{A(\alpha)}$, or is $\alpha$-indecomposable. If some $\alpha$-indecomposable $\gamma_{k}$ is not $\alpha$-minimal, then we can replace $\gamma_{k}$ with a directed path $\gamma_{k}^{\prime}$ such tht $\partial \gamma_{k}^{\prime}=\partial \gamma_{k}$, and where $\gamma_{k}^{\prime}$ is a concatenation of $\alpha$-minimal paths and paths in $\Gamma_{A(\alpha)}$. Thus, we can assume that each $\alpha$-indecomposable $\gamma_{k}$ is actually $\alpha$-minimal. Now alter the notation, setting $\gamma_{1}=\gamma_{a}$ and $\gamma_{K}=\gamma_{b}$. Let $\left(\gamma_{k(i)}\right)_{i=1, \ldots, I}$ be the subsequence of all $\alpha$-minimal paths in the sequence $\left(\gamma_{k}\right)_{k=1, \ldots, K}$; note that $k(1)=1$ and $k(I)=K$. Note also that Head $\left(\gamma_{k(i)}\right)$ and Tail $\left(\gamma_{k(i+1)}\right)$ are in the same component of $\Gamma_{A(a)}$, for each $i \in 1, \ldots, I$. Thus, $\left[\gamma_{k(1)}\right] *\left[\gamma_{k(2)}\right] * \cdots *\left[\gamma_{k(I)}\right]$ is a directed path in $\Gamma^{\prime}(\alpha)$ starting with $\left[\gamma_{a}\right]$ and ending with $\left[\gamma_{b}\right]$.

So to prove that $\Gamma_{\alpha}$ is strongly connected and $V_{\alpha}$ is positive, it suffices to prove:

Leмma 8.6. Construction of $\Gamma_{\alpha}$ and $V_{\alpha}$. There is an isomorphism from $\Gamma_{\alpha}$ to $\Gamma_{\alpha}^{\prime}$ such that $V_{\alpha}^{\prime} \in H^{1}\left(\Gamma_{\alpha}^{\prime} ; \mathbb{Z}\right)$ pulls back to $V_{\alpha} \in H^{1}\left(\Gamma_{\alpha} ; \mathbb{Z}\right)$.

Proof. Consider the directed graph $\tilde{\Gamma}_{\alpha}^{\prime}$ obtained as the $\mathbb{Z}$-cover of $\Gamma_{\alpha}^{\prime}$ with respect to the class $V_{\alpha}^{\prime}$. Then the lemma will be proved if we can exhibit a $\mathbb{Z}$-equivariant isomorphism between $\tilde{\Gamma}_{\alpha}$ and $\tilde{\Gamma}_{\alpha}^{\prime}$.

Let $\tilde{S}$ denote the inverse image under the projection $\tilde{M} \rightarrow M$ of the distinguished section $S$ of $\phi$, and let $\tilde{f}: \tilde{S} \rightarrow \tilde{S}$ be the first return map of $\tilde{\phi}$. Let $\mathcal{N}$ denote the 
collection of components of inverse images of elements of $\mathcal{M}$. Thus, $\mathcal{N}$ is a Markov partition for $\tilde{f}$. Let $\tilde{A}: \mathcal{N} \times \mathcal{N} \rightarrow\{0,1\}$ be the transition matrix. If $\mathcal{N}^{+}=\left\{B^{+} \mid B \in \mathcal{N}\right\}$, then $\mathcal{N}^{+}$is just the collection of lifts of all Markov flow boxes for the flow $\phi$.

Notice that there is a shift map $\tilde{\rho}$ on the shift space $S_{\tilde{A}}$, and a semi-conjugacy $\tilde{q}$ from the suspension flow of $\tilde{\rho}$ to $\tilde{\phi}$. Notice also that the $\mathbb{Z}$-action on $\tilde{M}$ induces natural $\mathbb{Z}$-actions on all of this data.

Let $\Gamma_{\tilde{A}}$ be the topological realization of $\tilde{A}$. Define a directed graph $\mathscr{G}$ whose nodes are the non-trivial strong components of $\Gamma_{\tilde{A}}$, with a directed edge from a node $G$ to a node $G^{\prime}$ if: there exists a directed path in $\Gamma_{\tilde{A}}$ from $G$ to $G^{\prime}$, and no such path passes through a strong component of $\Gamma_{\tilde{A}}$ distinct from $G$ and $G^{\prime}$. Notice that there is a $\mathbb{Z}$-covering map $\Gamma_{\tilde{A}} \rightarrow \Gamma_{A}$ corresponding to the cohomology class $U_{\alpha} \in H^{1}\left(\Gamma_{A} ; \mathbb{Z}\right)$. It is evident that $\Gamma_{\tilde{A}} \rightarrow \Gamma_{A}$ induces a $\mathbb{Z}$-covering map $\mathscr{G} \rightarrow \Gamma_{\alpha}^{\prime}$ corresponding to the cohomology class $V_{\alpha}^{\prime} \in H^{1}\left(\Gamma_{\alpha}^{\prime} ; \mathbb{Z}\right)$. Thus, it suffices to exhibit a $\mathbb{Z}$-equivariant isomorphism between the digraphs $\tilde{\Gamma}_{\alpha}$ and $\mathscr{G}$.

As is evident from the definition, the digraph structure on $\tilde{\Gamma}_{\alpha}$ is completely determined by the induced partial order on its nodes. The same is evidently true of $\mathscr{G}$. Thus, it suffices to exhibit a $\mathbb{Z}$-equivariant order isomorphism between $\mathscr{C}=$ Nodes $\left(\tilde{\Gamma}_{\alpha}\right)$ and Nodes $(\mathscr{G})$.

Consider $G \in \operatorname{Nodes}(\mathscr{G})$, i.e. $G$ is a strong component of $\Gamma_{\tilde{A}}$. Let $C(G)$ denote the closed invariant set of the flow $\tilde{\phi}$ consisting of all orbits of the form $O(\underline{m})$ for $\underline{m} \in S_{\tilde{A}}$, where $\underline{m}$ corresponds to a bi-infinite directed path in $G$. Since $G$ is strongly connected, evidently $C(G)$ is a chain connected invariant set of $\tilde{\phi}$, so $C(G)$ is contained in some chain component of $R(\tilde{\phi})$. We want to show moreover that $C(G)$ is a chain component of $R(\tilde{\phi})$, i.e. an element of $\mathscr{C}$. Under the projection map $p: \tilde{M} \rightarrow M$ and the corresponding projection $p: \Gamma_{\tilde{A}} \rightarrow \Gamma_{A}$, note that $p(G)$ is a component of $\Gamma_{A(\alpha)}$, and $p(C(G))$ is the corresponding invariant set of $\phi$. From Theorem 3.8 Characterization of the Chain Kernel of $\alpha$, together with Proposition 3.6 Characterization of $A(\alpha)$ and the special assumption that $R(\alpha)=L(\alpha)$, it follows that $p(C(G))$ is a chain component of $R(\alpha)$. From Proposition 7.1 Transient Behaviour of $\tilde{\phi}$, it follows that $C(G)$ is a chain component of $R(\tilde{\phi})$.

We have defined a map $G \rightarrow C(G)$ from Nodes $(\mathscr{G})$ to $\mathscr{C}$, which is evidently $\mathbb{Z}$-equivariant, and from the propositions mentioned in the previous paragraph, together with the definition of $\mathscr{G}$, it easy to see that this map is a bijection. To see that it preserves partial order, suppose $G<G^{\prime}$ in Nodes $(\mathscr{G})$. Thus, there is a directed path $g$ from $G$ to $G^{\prime}$ in $\Gamma_{\tilde{A}}$. Extend $g$ to a bi-infinite path $\gamma$, adding on a backwards half infinite path in $G$ to the left, and a forwards half infinite path in $G^{\prime}$ to the right. $\gamma$ corresponds to a symbolic sequence $\underline{m} \in S_{\tilde{A}}$, and $O(\underline{m})$ is an orbit whose $L_{-}$limit set is in $C(G)$, and whose $L_{+}$limit set is in $C\left(G^{\prime}\right)$. Evidently $O(\underline{m})$ is not contained in $R(\tilde{\phi})$, since $C(G)$ and $C\left(G^{\prime}\right)$ are distinct chain components of $R(\tilde{\phi})$. Thus, $C(G)<C\left(G^{\prime}\right)$ in $\mathscr{C}$.

This finishes the proof that $\Gamma_{\alpha}$ is strongly connected and $V_{\alpha}$ is positive in the case when $R(\alpha)$ contains no singular orbits of $\phi$. We shall say a few words about what happens in the other case. Again, the idea is to use the symbolic dynamics to construct a particular digraph for which strong connectivity is obvious, and then to 
build an isomorphism with $\Gamma_{\alpha}$. To do this, we again employ the notion of a pseudo-Markov partition $\mathcal{M}^{p}$ for $f$, with a Markov $2 n$-gon around any $s \in \operatorname{Sing}(f, n)$ such that the $\alpha$ vanishes on the periodic orbit of $\phi$ passing through $s$. Let $A^{p}: \mathscr{M}^{p} \times$ $\mathscr{M}^{p} \rightarrow\{0,1\}$ be the transition matrix, with topological realization $\Gamma^{p}$, and let $U^{p} \in$ $H^{1}\left(\Gamma^{p} ; \mathbb{Z}\right)$ be the cohomology class such that for each symbolic loop $\underline{m}$ of $A^{p}$, $U^{p}(\gamma(\underline{m}))=\langle\alpha, O(\underline{m})\rangle$. Now construct a digraph $\Gamma_{\alpha}^{\prime p}$ and class $V_{\alpha}^{\prime p}$ from $\Gamma^{p}$ and $U^{p}$, just as in the non-singular case $\Gamma_{\alpha}^{\prime}$ and $V_{\alpha}^{\prime}$ was constructed from $\Gamma_{A}$ and $U$. Again from the construction and strong connectivity of $\Gamma^{p}$, it is evident that $\Gamma_{\alpha}^{\prime p}$ is strongly connected and $V_{\alpha}^{\prime p}$ is positive. The same proof as before shows that $\Gamma_{\alpha}^{\prime p}$ is isomorphic to $\Gamma_{\alpha}$, and the isomorphism pulls $V_{\alpha}$ back to $V_{\alpha}^{\prime p}$.

\section{9. $\mathbb{Z}$-Spectral decomposition for basic sets of axiom $A$ flows}

We shall indicate briefly to what extent our results can be extended to basic sets of axiom $\boldsymbol{A}$ flows, which includes the case of transitive Anosov flows.

Let $M$ be a compact manifold, $\phi$ an Axiom $A$ no cycles flow on $M$. According to the Spectral Decomposition Theorem of Smale [SM], $R(\phi)$ has finitely many chain components, called basic sets of $f$. According to Bowen [B2], each basic set $I$ is the image, under a finite-to-one semi-conjugacy, of the suspension flow of a sub-shift of finite type; in fact, $I$ has a Markov family of local sections (Theorem 2.5 of [B2]). Thus, there is a directed graph $\Gamma_{I}$ encoding the symbolic dynamics of $\phi \mid I$.

Consider a basic set $I$, and a primitive cohomology class $\alpha \in H^{1}(M ; \mathbb{Z})$. Let $\tilde{M} \rightarrow M$ be the associated $\mathbb{Z}$-cover, $\tilde{\phi}$ the lifted flow on $\tilde{M}$ and $\tilde{I}$ the total lift of $I$. Let $\tilde{M}^{c}=\tilde{M} \cup\{+\infty,-\infty\}$ be the end compactification, $\tilde{\phi}^{c}$ the extended flow. We say that $\tilde{I}$ has a $\mathbb{Z}$-spectral decomposition if the following conditions hold:

(A) Each chain component of $R=R(\tilde{\phi} \mid \tilde{I})$ is compact;

(B) There are finitely many orbits of chain components of $R$ under the action of $\mathbb{Z}$;

(C) For any $x \in \tilde{I}-R$, either $L_{+}(x)=\{+\infty\}$ or $L_{+}(x)$ is contained in some chain component of $R$;

(D) Similarly, for any $x \in \tilde{I}-R$, either $L_{-}(x)=\{-\infty\}$ or $L_{-}(x)$ is contained in some chain component of $R$;

(E) If $L_{+}(x) \neq\{+\infty\}$, then there exists a neighborhood $U$ of $+\infty$ such that for any chain component $C$ of $R$, if $C \subset U$ then $C \subset R_{+}(x)$; a similar statement holds when $L_{-}(x) \neq\{-\infty\}$.

As mentioned in the introduction, the results of the companion paper [M] show that a necessary condition for $\tilde{I}$ to have a $\mathbb{Z}$-spectral decomposition is that $-\infty$ and $+\infty$ be in distinct components of the chain recurrent set of $\tilde{\phi}^{\mathrm{c}}$. This has consequences for the sign of $\alpha$ on homology directions:

Proposition 9.1. If $-\infty$ and $+\infty$ are in distinct components of the chain recurrent set of $\tilde{\phi}^{c} \mid \tilde{I}$, then $\alpha$ is either non-negative or non-positive on $D_{\phi}(I)$.

Proof. We shall prove this for $\tilde{I}=\tilde{M}$; the general case is no different. Let $\tilde{M}$ be equipped with an equivariant metric $d$; let $\tilde{M}^{\mathrm{c}}=\tilde{M} \cup\{-\infty,+\infty\}$ be equipped with any metric $d^{\text {c }}$. Let $\tau_{n}: \tilde{M} \rightarrow \tilde{M}$ denote the $\mathbb{Z}$-action.

Suppose that $\alpha$ assumes both positive and negative values on $D_{\phi}$. Using positivity, we shall show that $+\infty \in R_{+}(-\infty)$; a similar argument using negativity shows that $-\infty \in R_{+}(+\infty)$. It follows that $-\infty$ and $+\infty$ are in the same chain component of $R(\tilde{\phi})$. 
Let $\varepsilon, T$ be given. Using the metric $d^{\mathrm{c}}$, we must construct an $\varepsilon, T$ chain from $-\infty$ to $+\infty$. Let $U_{-}=\left\{x \in \tilde{M} \mid d^{\mathrm{c}}(x,-\infty)<\varepsilon\right\}$, and $U_{+}=\left\{x \in \tilde{M} \mid d^{\mathrm{c}}(x,+\infty)<\varepsilon\right\}$. Clearly $U_{-}$and $U_{+}$are open sets in $\tilde{M}$ and their common complement $\tilde{M}-$ $\left(U_{-} \cup U_{+}\right.$) is compact. Thus, there exists a number $\varepsilon^{\prime}$ such that any $d, \varepsilon^{\prime}$-ball centered on a point in $\tilde{M}-\left(U_{-} \cup U_{+}\right)$is contained in the $d^{c}, \varepsilon$ ball centered on the same point. Using the metric $d$, we need only exhibit an $\varepsilon^{\prime}, T$ chain from a point in $U_{-}$to a point in $U_{+}$. Henceforth, we shall drop the prime symbol from $\varepsilon^{\prime}$, and we shall assume that all distances and balls are measured with the metric $d$.

Choose a number $\eta>0$ so that the diameter of $\tilde{M}-\left(U_{-} \cup U_{+}\right)$is less than $\eta$. Choose a positive integer $N$ such that $\tau_{N}\left(\tilde{M}-U_{-}\right) \subset U_{+}$. We can assume that $\varepsilon$ is small enough so that $U_{-}$and $U_{+}$are disjoint; so if $y \in U_{+}$and $d(y, z)<\eta$, it follows that $\tau_{N}(z) \in U_{+}$.

Let $X_{i}=\left(x_{i}, t_{i}\right)$ be a closing sequence in $M$ such that the projective homology classes $p\left(X_{i}\right)$ approach some class $d \in H_{1}(M ; \mathbb{R}) / \mathbb{R}_{+}$for which $\alpha(d)>0$.

Suppose first that $\alpha\left(X_{i}\right)$ is unbounded; in this case, we actually construct a flow segment $\tilde{x} \cdot[0, t]$ with $t>T$ from $\tilde{x} \in U_{-}$to $\tilde{x} \cdot t \in U_{+}$. Choose $i$ so that $t_{i}>T$, $\alpha\left(X_{i}\right)=K>2 \cdot N+1$, and $d\left(x_{i}, x_{i} \cdot t_{i}\right)<\eta$. Choose $\tilde{x}$ to be a lifting of $x_{i}$ so that $\tilde{x} \in U_{-}$, and $\tau_{n}(\tilde{x}) \notin U_{-}$for all $n>0$. To show that $\tilde{x} \cdot\left[0, t_{i}\right]$ is the required flow segment, we need only show that $\tilde{x} \cdot t_{i} \in U_{+}$. Since $\tau_{1}(\tilde{x}) \in \tilde{M}-U_{-}$, and $K-N>$ $N+1$, then $\tau_{K-N}(\tilde{x}) \in U_{+}$. Clearly $d\left(\tau_{2 K}(\tilde{x}), \tilde{x} \cdot t_{i}\right)<\eta$, so $d\left(\tau_{K-N}(\tilde{x}), \tau_{-N}\left(\tilde{x} \cdot t_{i}\right)\right)<$ $\eta$, and it follows that $\tilde{x} \cdot t_{i}=\tau_{N}\left(\tau_{-N}\left(\tilde{x} \cdot t_{i}\right)\right) \in U_{+}$.

On the other hand, suppose that $\alpha\left(X_{i}\right)$ is bounded; since $\alpha(d)>0$, then the sequence of integers $\alpha\left(X_{i}\right)$ is eventually positive. Choose $\tilde{x}$ as in the previous paragraph. Choose $i$ so that $t=t_{i}>T, \alpha\left(X_{i}\right)=K>0$, and $d\left(x_{i}, x_{i} \cdot t\right)<r$, where $r$ is chosen to satisfy the following: defining $\tilde{x}_{0}=\tilde{x}$ and $\tilde{x}_{n}=\tau_{K}\left(\tilde{x}_{n-1}\right)$ for $n \geq 1$, then for every $n=1, \ldots, N, d\left(\tilde{x}_{n}, \tilde{x} \cdot K t\right)<\eta$. From this, an argument similar to that in the previous paragraph shows that, for any integer $L>(2 \cdot N+1) / K$, $\left(\tilde{x}_{0}, t ; \tilde{x}_{1}, t ; \ldots ; \tilde{x}_{L}, t\right)$ is an $\varepsilon, T$ chain with $\tilde{x}_{L} \in U_{+}$.

When $\tilde{I}$ has a $\mathbb{Z}$-spectral decomposition, it is evident that there exist points $x \in \tilde{M}$ such that $L_{+}(x)=+\infty$. From this it is easy to see that $\alpha$ cannot be identically zero of $D_{\phi}(I)$. Together with Proposition 9.1, it follows that a necessary condition for $\tilde{I}$ to have a $\mathbb{Z}$-spectral decomposition is that $\alpha$ be non-negative but not strictly zero on $D \phi(I)$.

We are interested in knowing whether these conditions are also sufficient: if $\alpha$ is non-negative but not strictly zero on $D_{\phi}(I)$, does $\tilde{I}$ have a $\mathbb{Z}$-spectral decomposition? Some of the techniques of $\S \S 3-8$ extend to the more general setting of basic sets of Axiom $A$ flows, as long as the definitions are suitably restated. The chain kernel of $\alpha$ restricted to $I, R(\alpha ; I)$, is defined as the set of points $x \in I$ such that for all $\varepsilon, T$ there exists an $\varepsilon, T$ cycle $X$ through $x$ such that $\langle\alpha, X\rangle=0$. The techniques of § 3-5 apply to the analysis of $R(\alpha ; I)$ without the difficulties that occur in the pseudo-Anosov case: the standard shadowing arguments work perfectly well in this case. Thus, there exists a sub-digraph $\Gamma_{I, \alpha} \subset \Gamma$ such that $R(\alpha ; I)$ consists of the union of orbits represented by a symbol sequence from the digraph $\Gamma_{l, \alpha}$. 
The main difficulty incurred is in attempting to generalize the results of $\S 6$, in which it is shown that the chain kernel always lifts to $\tilde{M}$. The main technique used there was to construct a neighborhood of the chain kernel to which the standard lifting lemma applies. We abstract the required property for such a neighborhood with the following definition: given a compact isolated invariant set $K$ of a flow $\phi$, $K$ is said to be homologically taut if there exists an isolating block $N$ for $K$ such that the image of the inclusion induced map $H_{1}(N ; \mathbb{R}) \rightarrow H_{1}(M ; \mathbb{R})$ is generated by Cone $\left(D_{\phi}(K)\right)$; the isolating block $N$ is said to be a homologically taut neighborhood. The main question that needs to be addressed in order to solve the lifting problem is:

Question. If $K$ is a hyperbolic invariant set, is $K$ homologically taut?

The proof given in $\S 6$ in the pseudo-Anosov case for $K=R(\alpha)$ does not always work in the Axiom $A$ case for $K=R(\alpha ; I)$. In the proof of $\S 6$, we constructed a homologically taut neighborhood from a Markov family of local sections $\mathcal{M}^{\prime}$ for $R(\alpha)$; the Markov rectangles in $\mathcal{M}^{\prime}$ were obtained by shrinking the rectangles corresponding to vertices of the digraph $\Gamma_{l, \alpha}$, and $N$ was then obtained by erecting a 'skewed box' $D(B)$ based at each element $B \in \mathcal{M}^{\prime}$. What made this neighborhood homologically taut was the fact that there were no identifications among the sides of flow boxes $B^{+}$over elements $B \in M^{\prime}$, so the skewed boxes $D(B)$ could be constructed with no side identifications; Thus, $H_{1}(N ; \mathbb{R})$ had no extraneous homology classes.

A general hyperbolic invariant set $K$ will not possess a Markov family of local sections with the required properties. However, there is one special case which works: Bowen has proven that if $K$ is a 1-dimensional hyperbolic invariant set, then $K$ is not just semi-conjugate, but actually conjugate to a subshift of finite type. In the course of the proof, Bowen constructs a Markov family of local sections $\mathcal{M}$ for $K$ to which the above construction applies (see [B1]). Thus, we have:

Proposition 9.2. A 1-dimensional hyperbolic invariant set is homologically taut.

It seems difficult to tell in general whether a hyperbolic invariant set is homologically taut. Perhaps one can apply the techniques of $\S 5$ of [B2], in which the boundary identifications among flow boxes are analyzed by building a hierarchy of subshifts.

Returning to the main course of the discussion, if one can prove that $R(\alpha ; I)$ is homologically taut, then the lifting problem of $\S 6$ can be solved.

Once the lifting problem is solved, the techniques of $\S \S 7$ and 8 apply exactly as stated; so it follows that $\tilde{I}$ has a $\mathbb{Z}$-spectral decomposition. Coupled with Proposition 9.2, we therefore have the following generalization of the $\mathbb{Z}$-Spectral Decomposition Theorem:

THEOREM 9.3. If $R(\alpha ; I)$ is homologically taut, then İ has a $\mathbb{Z}$-spectral decomposition. Thus, if $R(\alpha ; I)$ is 1 -dimensional, then $\tilde{I}$ has a $\mathbb{Z}$-spectral decomposition. In particular, if $I$ is 1-dimensional, then for any $\alpha \in H^{1}(M ; \mathbb{Z})$ which is non-negative but not identically zero on $D_{f}(I), \tilde{I}$ has a $\mathbb{Z}$-spectral decomposition. 


\section{REFERENCES}

[B1] R. Bowen. One dimensional hyperbolic sets for flows. J. Differential Equations 12 (1972), 173-179.

[B2] R. Bowen. Symbolic dynamics for hyperbolic flows. Amer. J. Math. 95 (1973), 429-460.

[C] C. Conley. Isolated invariant sets and the Morse index. CBMS Regional Conference Series in Math. 38 (1978).

[CE] C. Conley \& B. Easton. Isolated invariant sets and isolating blocks. Trans. AMS 158 (1) (1971), 35-61.

[FLP] Fathi, Laudenbach, Poenaru et al. Travaux de Thurston sur les Surfaces. Astérisque 66-67 (1979).

[F1] D. Fried. Fibrations over $S^{1}$ with pseudo-Anosov monodromy, exposé 14 of Travaux de Thurston sur les surfaces. Astérisque 66-67 (1979).

[F2] D. Fried. The geometry of cross sections to flows. Topology 21 (4) (1982), 353-371.

[G] D. Gabai. Foliations and the topology of 3-manifolds. J. Diff. Geom. 18 (1983), 445-503.

[H1] M. Handel. Global shadowing of pseudo-Anosovs. Ergod. Th. \& Dynam. Sys. 5 (1985), 373-377.

[H2] M. Handel. Entropy and semi-conjugacy in dimension two. Ergod. Th. \& Dynam. Sys. 8 (1988) 585-596.

[HW] G. H. Hardy \& E. M. Wright. An Introduction to the Theory of Numbers 5th ed. (Oxford University Press, 1979).

[M] L. Mosher. Surfaces and branched surfaces transverse to pseudo-Anosov flows on 3-manifolds. J. Diff. Geom., to appear.

[O] U. Oertel. Homology branched surfaces: Thurston's norm on $\mathbf{H}_{2}\left(\mathbf{M}^{3}\right)$. In: D. B. A. Epstein, ed., Low-dimensional Topology and Kleinian Groups. London Math. Soc. Lecture Notes Series 112 (1984), 253-272.

[Sch] S. Schwartzman. Asymptotic cycles. Ann. Math. 66 (1957), 270-284.

[Sh] M. Shub. Global stability of dynamical systems. (Springer Verlag: New York, 1987).

[Sm] S. Smale. Differentiable dynamical systems. Bull. Amer. Math. Soc. 73 (1967), 747-817.

[T] W. Thurston. A norm on the homology of 3-manifolds. Memoirs of the AMS 339 (1986). 